University of Massachusetts Amherst

ScholarWorks@UMass Amherst

Doctoral Dissertations

Dissertations and Theses

December 2020

\title{
AN INVESTIGATION OF FIT CRITERIA WITHIN MG-CFA FOR EXAMINING NON-NEGLIGIBLE MEASUREMENT INVARIANCE
}

Abdolvahab Khademi

University of Massachusetts Amherst

Follow this and additional works at: https://scholarworks.umass.edu/dissertations_2

Part of the Educational Assessment, Evaluation, and Research Commons, and the Educational Methods Commons

\section{Recommended Citation}

Khademi, Abdolvahab, "AN INVESTIGATION OF FIT CRITERIA WITHIN MG-CFA FOR EXAMINING NONNEGLIGIBLE MEASUREMENT INVARIANCE" (2020). Doctoral Dissertations. 2035.

https://doi.org/10.7275/19258798 https://scholarworks.umass.edu/dissertations_2/2035

This Open Access Dissertation is brought to you for free and open access by the Dissertations and Theses at ScholarWorks@UMass Amherst. It has been accepted for inclusion in Doctoral Dissertations by an authorized administrator of ScholarWorks@UMass Amherst. For more information, please contact scholarworks@library.umass.edu. 


\section{AN INVESTIGATION OF FIT CRITERIA WITHIN MG-CFA FOR EXAMINING NON- NEGLIGIBLE MEASUREMENT INVARIANCE}

A Dissertation Presented

by

ABDOLVAHAB KHADEMI SHAMAMI

Submitted to the Graduate School of the University of Massachusetts Amherst in partial fulfillment of the requirements for the degree of

DOCTOR OF PHILOSOPHY

September 2020

College of Education

Research, Educational Measurement, and Psychometrics 
(C) Copyright by Abdolvahab Khademi Shamami 2020

All Rights Reserved 


\section{AN INVESTIGATION OF FIT CRITERIA WITHIN MG-CFA FOR EXAMINING NON- NEGLIGIBLE MEASUREMENT INVARIANCE}

A Dissertation Presented

by

ABDOLVAHAB KHADEMI SHAMAMI

Approved as to style and content by:

Craig S. Wells, Chair

Scott Monroe, Member

Anna Liu, Member

Jennifer Randall

Associate Dean of Academic Affairs

College of Education 


\section{ACKNOWLEDGMENTS}

The Department of

Mathematics and Statistics

was the only place I felt

welcome and at home. I made

a lot of friends: classmates,

professors, and staff. All

lovely, hardworking, helpful

people.

Thank you! 


\section{ABSTRACT \\ AN INVESTIGATION OF FIT CRITERIA WITHIN MG-CFA FOR EXAMINING NON- NEGLIGIBLE MEASUREMENT INVARIANCE}

SEPTEMBER 2020

ABDOLVAHAB KHADEMI SHAMAMI, B.A., TEHRAN ISLAMIC AZAD UNIVERSITY

M.A., IRAN UNIVERSITY OF SCIENCE \& TECHNOLOGY

M.Sc. UNIVERSITY OF MASSACHUSETTS AMHERST

Ph.D., UNIVERSITY OF MASSACHUSETTS AMHERST

Directed by: Professor Craig S. Wells

One desirable property of a measurement process or instrument is the maximum invariance of the results across subpopulations with similar distribution of the traits. Determining measurement invariance (MI) is a statistical procedure in which different methods are used given different factors, such as the nature of the data (e.g. continuous, or discrete, completeness), sample size, measurement framework (e.g. observed scores, latent variable modeling), and other context-specific factors. To evaluate the statistical results, numerical criteria are often used, derived from theory, simulation, or practice. One statistical method to evaluate MI is multiple-group confirmatory factor analysis (MG-CFA) in which the amount of change in fit indices of nested models, such as comparative fit index (CFI), Tucker-Lewis fit index (TLI), and the root mean squared 
error of approximation (RMSEA), are used to determine if the lack of invariance is nontrivial. Currently, in the MG-CFA framework for establishing MI, the recommended effect size is a change of less than 0.01 in CFI/TLI measures (Cheung \& Rensvold, 2002). However, the recommended cutoff value is a very general index and may not be appropriate under some conditions, such as dichotomous indicators, different estimation methods, different sample sizes, and model complexity. In addition, in determining the cutoff value, consequences to the lack of invariance have been ignored in the current research. To address these gaps, the present research undertakes to evaluate the appropriateness of the current effect size of $\Delta \mathrm{CFI}$ or $\Delta \mathrm{TLI}<0.01$ in educational measurement settings, where the items are dichotomous, the item response functions follow an item response theory (IRT) model, estimation method is robust weighted least squares, and the focal and reference groups differ from each other on the IRT scale by 0.5 units (equivalent to \pm 1 raw score). A simulation study was performed with five (crossed) factors: percent of differential functioning items, IRT model, IRT $a$ and $b$ parameters, and the sample size. The results of the simulation study showed that the cutoff value of a $\Delta \mathrm{CFI} / \Delta \mathrm{TLI}<0.01$ for establishing $\mathrm{MI}$ is not appropriate for educational settings under the foregoing conditions. 


\section{TABLE OF CONTENTS}

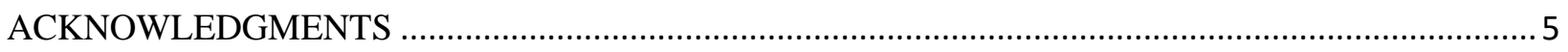

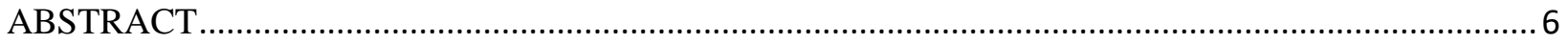

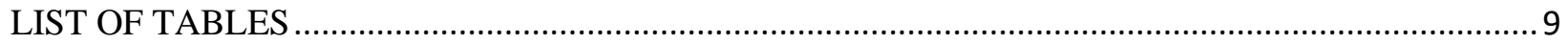

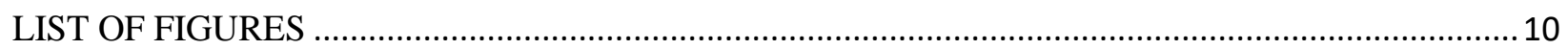

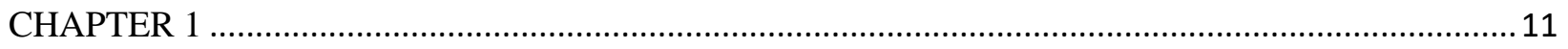

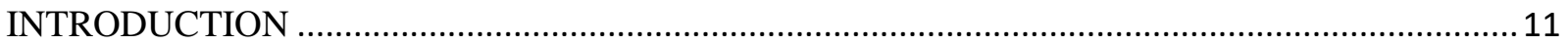

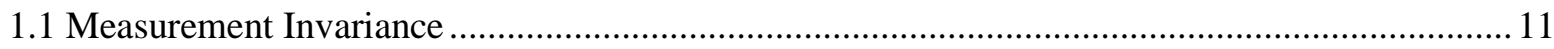

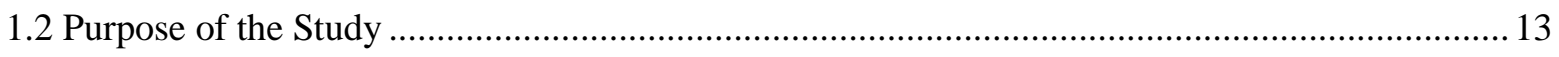

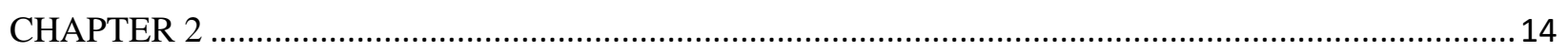

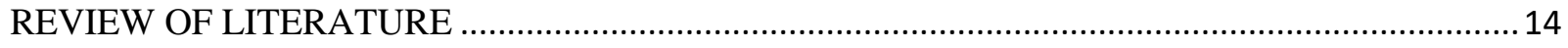

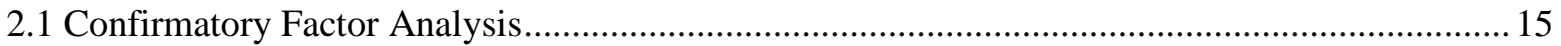

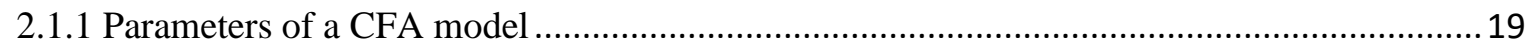

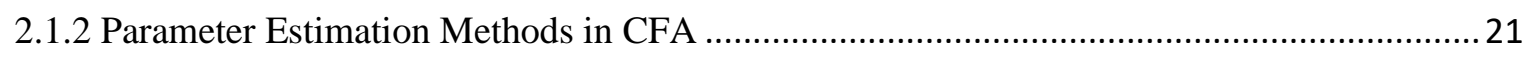

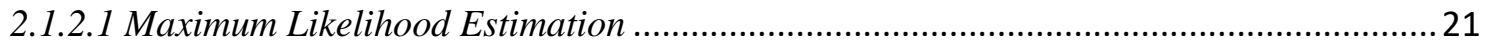

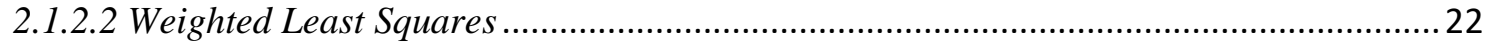

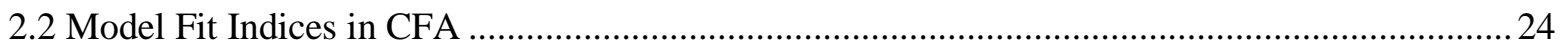

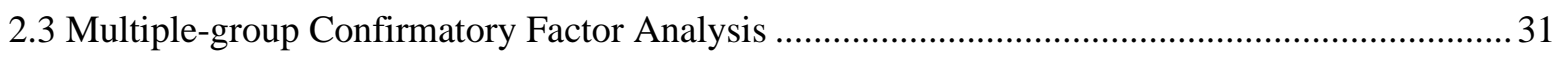

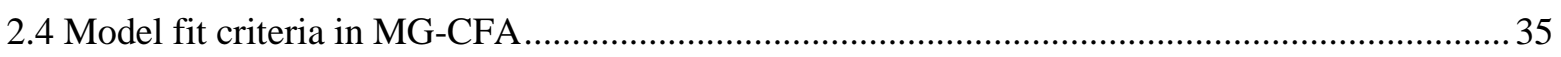

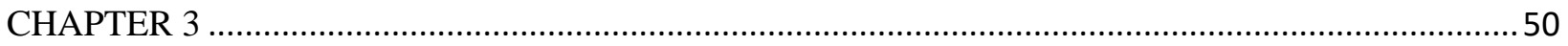

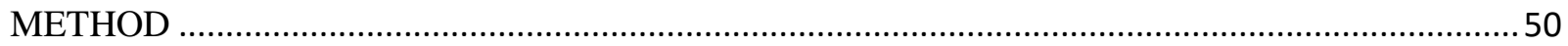

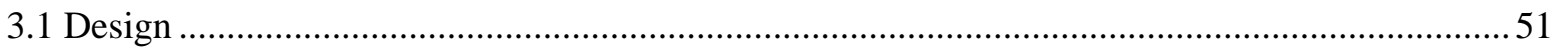

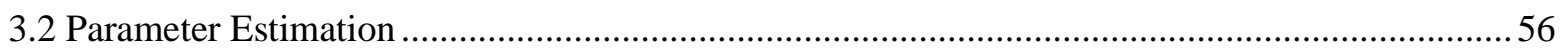

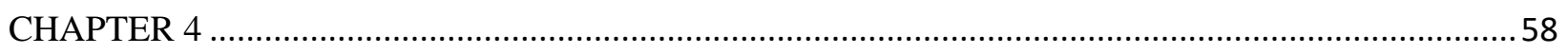

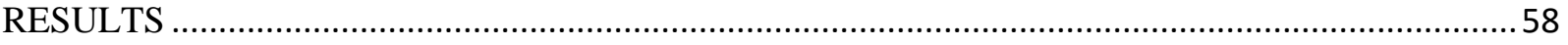

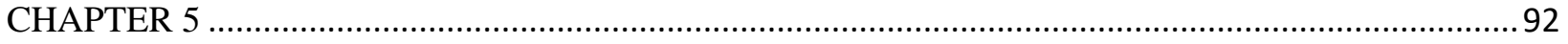

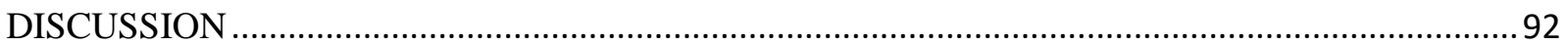


REFERENCES . 


\section{LIST OF TABLES}

2.The $b$ parameter values used to obtain LOI items in the focal group.

4.1. Proportions of Simulated DIF Items Detected by Mantel-Haenszel Test when IRT model $=2$ PL, DIF percentage $=20 \%$

4.2. Five-way ANOVA on CFI Change across the Five Factors .60

4.3. Five-way ANOVA on TLI Change across the Five Factors. .61

4.4. Five-way ANOVA on RMSEA Change across the Five Factors.

4.5. Change in Equal Loadings Equal Threshold (Scalar Invariance) GFI across conditions for $\mathrm{N}=500$, IRT $=3 \mathrm{PL}$

4.6. Change in Equal Loadings Equal Threshold (Scalar Invariance) GFI across conditions for $\mathrm{N}=500, \mathrm{IRT}=2 \mathrm{PL}$

4.7. Change in Equal Loadings Equal Threshold (Scalar Invariance) GFI's across conditions for $\mathrm{N}=1000$, IRT $=3 \mathrm{PL}$

4.8. Change in Equal Loadings Equal Threshold (Scalar Invariance) GFI's across conditions for $\mathrm{N}=1000$, IRT $=2 \mathrm{PL}$

4.9. Change in Equal Loadings Equal Threshold (Scalar Invariance) GFI's across conditions for $\mathrm{N}=2000$, IRT $=3 \mathrm{PL}$

4.10. Change in Equal Loadings Equal Threshold (Scalar Invariance) GFI's across conditions for $\mathrm{N}=2000$, IRT=2PL

4.11. Distribution statistics Standard Deviation (SD), Skewedness (SK), and Kurtosis (KU) for change in CFI, TLI, and RMSEA at scalar level across conditions for $\mathrm{N}=500$, $\mathrm{IRT}=3 \mathrm{PL}$

4.12. Distribution statistics Standard Deviation (SD), Skewedness (SK), and Kurtosis (KU) for change in CFI, TLI, and RMSEA at scalar level across conditions for $\mathrm{N}=500$, $\mathrm{IRT}=2 \mathrm{PL}$

4.13. Distribution statistics Standard Deviation (SD), Skewedness (SK), and Kurtosis (KU) for change in CFI, TLI, and RMSEA at scalar level across conditions for $\mathrm{N}=1000$, $\mathrm{IRT}=3 \mathrm{PL}$ 
4.14. Distribution statistics Standard Deviation (SD), Skewness (SK), and Kurtosis (KU) for change in CFI, TLI, and RMSEA at scalar level across conditions for $\mathrm{N}=1000, \mathrm{IRT}=2 \mathrm{PL}$

4.15. Distribution statistics Standard Deviation (SD), Skewness (SK), and Kurtosis (KU) for change in CFI, TLI, and RMSEA at scalar level across conditions for N= 2000, IRT $=3$ PL.........85

4.16. Distribution statistics Standard Deviation (SD), Skewness (SK), and Kurtosis (KU) for change in CFI, TLI, and RMSEA at scalar level across conditions for $\mathrm{N}=2000$, IRT $=2 \mathrm{PL}$.........88 


\section{LIST OF FIGURES}

2.1: A CFA model with two correlated factors

4.1: Change in equal loadings, equal threshold (scalar invariance) GFI's across conditions for $\mathrm{N}=500$, IRT $=3$ PL .63

4.2: Change in equal loadings, equal threshold (scalar invariance) GFI's across conditions for $\mathrm{N}=500$, IRT $=2 P L$ .66

4.3: Change in equal loadings equal threshold (scalar invariance) GFI's across conditions for $\mathrm{N}=1000, \mathrm{IRT}=3 \mathrm{PL}$

4.4: Change in equal loadings equal threshold (scalar invariance) GFI's across conditions for $\mathrm{N}=1000, \mathrm{IRT}=2 \mathrm{PL}$

4.5: Change in equal loadings equal threshold (scalar invariance) GFI's across conditions for $\mathrm{N}=2000, \mathrm{IRT}=3 \mathrm{PL}$

4.6: Change in equal loadings equal threshold (scalar invariance) GFI's across conditions for $\mathrm{N}=2000, \mathrm{IRT}=2 \mathrm{PL}$

4.7: Dispersion statistics ( $\mathrm{SD}=$ standard deviation, $\mathrm{SK}=$ skewedness, $\mathrm{KU}=$ kurtosis) for the distributions of the change in the CFI, TLI, and the RMSEA fit indices at scalar level across simulation conditions for $\mathrm{N}=500, \mathrm{IRT}=3 \mathrm{PL}$, DIF percentage $=10 \%$.

4.8: Dispersion statistics ( $\mathrm{SD}=$ standard deviation, $\mathrm{SK}=$ skewedness, $\mathrm{KU}=$ kurtosis) for the distributions of the change in the CFI, TLI, and the RMSEA fit indices at scalar level across simulation conditions for $\mathrm{N}=500$, IRT $=3 \mathrm{PL}$, DIF percentage $=20 \%$ .75

4.9: Dispersion statistics ( $\mathrm{SD}=$ standard deviation, $\mathrm{SK}=$ skewedness, $\mathrm{KU}=$ kurtosis) for the distributions of the change in the CFI, TLI, and the RMSEA fit indices at scalar level across simulation conditions for $\mathrm{N}=500$, IRT $=2 \mathrm{PL}$, DIF percentage $=10 \%$. .77

4.10. Dispersion statistics ( $\mathrm{SD}=$ standard deviation, $\mathrm{SK}=$ skewedness, $\mathrm{KU}=$ kurtosis) for the distributions of the change in the CFI, TLI, and the RMSEA fit indices at scalar level across simulation conditions for $\mathrm{N}=500$, IRT $=2 \mathrm{PL}$, DIF percentage $=20 \%$. 
4.11. Dispersion statistics ( $\mathrm{SD}=$ standard deviation, $\mathrm{SK}=$ skewedness, $\mathrm{KU}=$ kurtosis) for the distributions of the change in the CFI, TLI, and the RMSEA fit indices at scalar level across simulation conditions for $\mathrm{N}=1000, \mathrm{IRT}=3 \mathrm{PL}$, DIF percentage $=10 \%$.

4.12. Dispersion statistics ( $\mathrm{SD}=$ standard deviation, $\mathrm{SK}=$ skewness, $\mathrm{KU}=$ kurtosis) for the distributions of the change in the CFI, TLI, and the RMSEA fit indices at scalar level across simulation conditions for $\mathrm{N}=1000, \mathrm{IRT}=3 \mathrm{PL}$, DIF percentage $=20 \%$.

4.13. Dispersion statistics ( $\mathrm{SD}=$ standard deviation, $\mathrm{SK}=$ skewness, $\mathrm{KU}=$ kurtosis) for the distributions of the change in the CFI, TLI, and the RMSEA fit indices at scalar level across simulation conditions for $\mathrm{N}=1000, \mathrm{IRT}=2 \mathrm{PL}, \mathrm{DIF}$ percentage $=10 \%$.

4.14. Dispersion statistics ( $\mathrm{SD}=$ standard deviation, $\mathrm{SK}=$ skewness, $\mathrm{KU}=$ kurtosis) for the distributions of the change in the CFI, TLI, and the RMSEA fit indices at scalar level across simulation conditions for $\mathrm{N}=1000, \mathrm{IRT}=2 \mathrm{PL}$, DIF percentage $=20 \%$.

4.15. Dispersion statistics ( $\mathrm{SD}=$ standard deviation, $\mathrm{SK}=$ skewness, $\mathrm{KU}=$ kurtosis) for the distributions of the change in the CFI, TLI, and the RMSEA fit indices at scalar level across simulation conditions for $\mathrm{N}=2000$, IRT $=3 \mathrm{PL}, \mathrm{g}$ DIF percentage $=10 \%$.

Dispersion statistics ( $\mathrm{SD}=$ standard deviation, $\mathrm{SK}=$ skewness, $\mathrm{KU}=$ kurtosis) for the distributions of the change in the CFI, TLI, and the RMSEA fit indices at scalar level across simulation conditions for $\mathrm{N}=2000, \mathrm{IRT}=3 \mathrm{PL}$, DIF percentage $=20 \%$.

4.17. Dispersion statistics ( $\mathrm{SD}=$ standard deviation, $\mathrm{SK}=$ skewness, $\mathrm{KU}=$ kurtosis) for the distributions of the change in the CFI, TLI, and the RMSEA fit indices at scalar level across simulation conditions for $\mathrm{N}=2000, \mathrm{IRT}=2 \mathrm{PL}$, DIF percentage $=10 \%$. 89

4.18. Dispersion statistics ( $\mathrm{SD}=$ standard deviation, $\mathrm{SK}=$ skewness, $\mathrm{KU}=$ kurtosis) for the distributions of the change in the CFI, TLI, and the RMSEA fit indices at scalar level across simulation conditions for $\mathrm{N}=2000, \mathrm{IRT}=2 \mathrm{PL}$, DIF percentage $=20 \%$. 


\section{CHAPTER 1}

\section{INTRODUCTION}

\subsection{Measurement Invariance}

In educational and psychological assessments, it is often important to ensure that the intended construct has the same meaning for individuals from different groups or subpopulations (e.g., defined by gender or testing mode). In fact, one of the five sources of validity, internal structure, states that the assessment function the same across groups (American Educational Research Association, American Psychological Association, \& National Council on Measurement in Education, 2014). In addition, according to the Standards for Educational and Psychological Testing (ibid), construct-irrelevant factors such as test takers' linguistic and cultural characteristics (among others) may impede test takers in demonstrating their standing on the intended constructs. The Standards also advise test developers and psychometricians that subgroup differences may affect the reliability, validity, and comparability of score interpretations for the intended individuals. Therefore, measurement invariance, which states that a construct is being measured in the same way across groups, plays an important role in the development of educational assessments (Millsap, 2011).

One of the challenges in assessing measurement invariance is that it may be unrealistic to expect it to be strictly met, yet the test scores may be comparable-enough to support valid inferences. For example, when comparing examinees who took an assessment on a paper-based test to those who answered the items on the computer, one may expect measurement invariance to be violated (at least slightly), especially for items that are specifically made for the computer (e.g., technology enhanced items). In such a case, the important question is not whether strict measurement invariance holds, but whether the lack of invariance is negligible. 
There is a plethora of statistical methods available for assessing measurement invariance such as the Mantel-Haenszel test (Holland \& Thayer, 1988), likelihood-ratio test (Thissen, Steinberg, \& Gerrard, 1986; Thissen, Steinberg, \& Wainer, 1988, 1993), and multi-group confirmatory factor analysis (MG-CFA) (Muthen, 1989). The appealing features of MG-CFA are that it allows one to examine the measurement invariance in the context of a detailed dimensionality analysis, the model controls for the effect of measurement error, and the literature has suggested an effect size to judge the magnitude of a lack of measurement invariance. The purpose of the present study is to examine the effect size in MG-CFA for data from an educational test.

In MG-CFA, the parameters from a measurement model are estimated and compared across groups. In particular, one is interested in comparing the factor loadings (which represent item discrimination), thresholds (which represent item difficulty), and, to a lesser extent, residual variances (which represent measurement error of each item). The comparisons are conducted by first fitting an unrestricted model where the model parameters are freely estimated in each group, and then a restricted model is fit that imposes equality constraints on the parameter values across groups (e.g., factor loadings are constrained to be equal between the groups). To determine if measurement invariance holds, the fit of the unrestricted and restricted models is compared. Traditionally, the difference in $\chi^{2}$ fit statistics between the two models has been used to test the hypothesis that the parameter values are equal across groups; therefore, if the hypothesis is correct, the fit of the model should not get significantly worse when placing equality constraints on the model.

The problem with using the difference in $\chi^{2}$ statistics is that the traditional hypothesis being tested, which states that the item parameter values are identical between the groups, is not 
necessarily true since we do not expect measurement invariance to hold exactly. And, since MGCFA models are often fit to very large samples, the statistical tests have exceptional power to reject this false null hypothesis. One possible solution to this problem is to develop an effect size (ES) measure to indicate the practical significance of a possible lack of measurement invariance. One ES measure that has been developed and examined in the literature is the difference in goodness-of-fit indices, such as the comparative fit index (CFI), between the restricted and unrestricted models. One problem remains, however, and that is determining an appropriate cutoff value that indicates when a model lacks measurement invariance beyond a negligible amount.

\subsection{Purpose of the Study}

Although a decrease in goodness-of-fit indices that exceeds 0.01 as a sign of violation of invariance has been suggested in the literature (Cheung \& Rensvold, 2002; Meade, Johnson, \& Braddy, 2008), it is unclear if that value is appropriate for educational tests. For example, the previous research was conducted using indicators that are not based on item responses from common educational assessments (e.g., dichotomous indicators). Second, and perhaps more importantly, the cutoff value was not attached to a consequence of violating measurement invariance. Therefore, one of the purposes of the present study is to evaluate the current cutoff criterion in practice and to determine an appropriate cutoff value when testing measurement invariance in which the consequences of violating measurement invariance are defined within an educational testing context. A secondary purpose of the study is to examine whether the distributions of the effect sizes are influenced by various factors such as item discrimination and the percentage of items exhibiting a lack of measurement invariance. 


\section{CHAPTER 2}

\section{REVIEW OF LITERATURE}

A desirable property of an instrument is that individuals with the same observed score have the same standing on the construct underlying the instrument (Millsap, 2011; Schmitt \& Kuljanin, 2008). In other words, if the respondents have the same standing on the construct continuum, the instrument must show equal or invariant measurement or psychometric properties across groups. If we denote the observed score as $\mathrm{X}$ and the latent variable as $\mathrm{W}$, then measurement invariance (MI), "expresses the idea that the measurement properties of $\mathrm{X}$ in relation to the target latent trait $\mathrm{W}$ are the same across the populations" (Millsap, 2011, p. 46).

One rationale for investigating measurement invariance can be attributed to the increased group membership diversity (Schmitt \& Kuljanin, 2008). Among others, the groups may be different from each other in terms of ethnic, racial, linguistic, age, assessment mode (e.g., computer-based versus paper-and-pencil formats), and time points. In fact, comparison of subpopulations on an instrument may be meaningless unless measurement invariance is reasonably established (Cheung \& Rensvold, 2002; Schmitt \& Kuljanin, 2008).

Measurement non-invariance or lack of invariance (LOI) is a type of systematic error introduced in the relationship between the latent factor and the manifest indicator (Vandenberg $\&$ Lance, 2000). Establishing measurement invariance is part of evaluating measurement quality and the violation of measurement invariance assumptions threatens the substantive interpretation of the observed scores (Vandenberg \& Lance, 2000). Therefore, establishing measurement invariance is a logical prerequisite to the evaluation of substantive hypotheses across groups (ibid). As for the importance of meeting measurement invariance in a test, Standards 3.2 and 3.17, respectively, of the Standards for Educational and Psychological Testing (American Educational Research Association, American Psychological Association, \& National Council on 
Measurement in Education, 2014) require evidence that the assessments are performing comparably in different groups, as indicated by the following two quotes:

Test developers are responsible for developing tests that measure the intended construct and for minimizing the potential for tests' being affected by construct-irrelevant characteristics, such as linguistic, communicative, cognitive, cultural, physical, or other characteristics (p. 64).

When aggregate scores are publicly reported for relevant subgroups--for example, males and females $[\ldots]$--test users are responsible for providing evidence of comparability and for including cautionary statements whenever credible research or theory indicates that test scores may not have comparable meaning across these subgroups (p. 71).

Although there is a plethora of statistical methods available for assessing measurement invariance, one commonly used method within structural equation modeling (SEM) is multiple group confirmatory factor analysis (MG-CFA). The following sections will provide a detailed description of MG-CFA, how it is used to assess measurement invariance using fit indices, and the current research studies that have explored the use of fit indices in MG-CFA.

\subsection{Confirmatory Factor Analysis}

Confirmatory factor analysis (CFA) belongs to the family of factor analysis procedures where the goal is to reduce the data to the principal variables or dimensions that in a parsimonious manner describe the structure of the observed data suggested by theory or 
hypothesis. In other words, CFA delineates how observed indicators represent certain latent variables (Thompson, 2004).

One major application of CFA is evaluating the psychometric properties of educational tests. CFA is used in the psychometric evaluation of tests and instruments in several ways. For instance, CFA is used to establish the number of latent traits in an instrument (dimension analysis) and the pattern of item-factor relationships. As mentioned above, CFA is theory-driven in that the researcher starts out with a theoretical structure of the constructs, including the number of latent traits, and then fits a model to verify that such a theoretical structure exists. In addition, CFA can reveal the relationship between the constructs and the indicators in terms of factor loadings. The relationship between a latent variable and its indicators is also used for producing discriminant or convergent validity evidence required in construct validation of an instrument. Another application of CFA in construct validation is the analysis of multitraitmultimethod matrices, in which the resulting estimates of convergent and divergent validity are adjusted for measurement error and error theory (Brown, 2006).

The dimensionality analysis results obtained using CFA can also be used for scoring purposes, in that subscales may be needed if factors represent different domains of a single construct. In addition, CFA can produce scale reliability of an instrument that outperforms traditional methods such as coefficient alpha (Brown, 2006). CFA may also be used in detecting method effects (additional covariation among indicators induced by the measurement approach employed) both across methods and within a method using the error theory of the measurement model.

In CFA, the researcher posits a hypothetical dimensional structure and then models that factor structure against observed data in the form of multiple indicators or items. Figure 1 
illustrates a hypothetical two-factor CFA model in which each indicator is regressed on one of the latent variables.

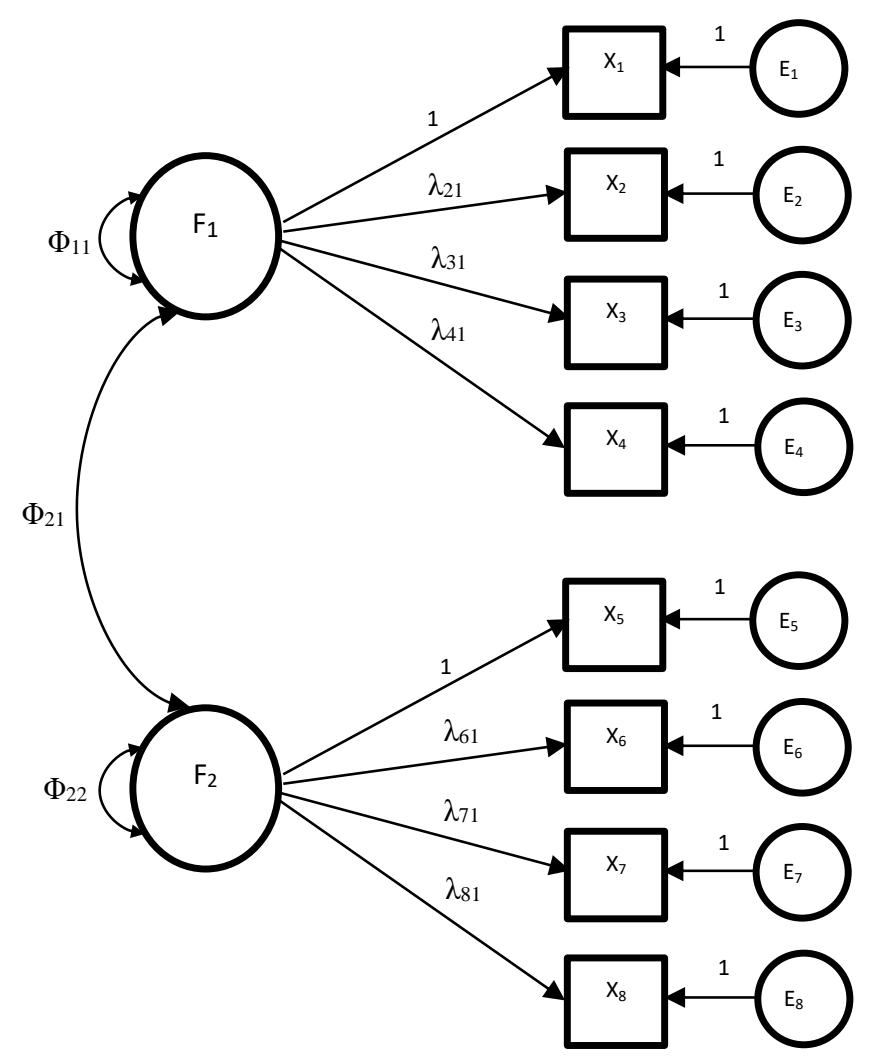

Figure 2.1: A CFA model with two correlated factors.

The observed data are usually transformed into a variance-covariance matrix (in contrast to EFA, where a correlation matrix is constructed). One of the goals of CFA is to determine if the factor structure defined by the model can adequately reproduce the observed covariance matrix.

CFA is based on the common factor model, which also underpins exploratory factor analysis (EFA). The central theme in factor analysis is the existence of a latent trait or factor that accounts for variation or covariation among a set of indicators. The indicators are correlated because they share a common source of variation without which they become uncorrelated (in the absence of construct-irrelevant factors). This shared variance is called the common factor. 
Specifically, the common factor model, introduced by Thurstone (1947), postulates that the value of each indicator is a linear function of one or more common factors and one unique factor (residual). Therefore, in partitioning of the covariance matrix, factor analysis extracts common variance (due to the common factor) and unique variance (due to other factors and random error). The common factor model can be mathematically expressed as follows:

$$
y=\Lambda_{y} \eta+\varepsilon
$$

where $y$ is an indicator, $\Lambda$ is the factor loading, $\eta$ is a latent factor and $\varepsilon$ is the unique variance. A factor loading indicates the strength of the relationship between the indicator and the factor. A factor loading is analogous to regression weight or slope in regression analysis and can be interpreted similar to the slope coefficients in a multiple linear regression or the correlation coefficients in correlation analysis. The squared form of standardized factor loading provides a measure of explained variance, often called communality in factor analysis.

The unique variance is analogous to the residuals in the regression analysis, which includes all sources of variation not accounted for by the common factor. The other sources of variation could be systematic errors such as other factors, method variance, and random errors. In CFA, the error terms can be correlated or not, based on theory (i.e. error theory). When error terms are specified to be correlated in CFA, it is assumed that part of their covariation emanates from sources other than the common factor (Brown, 2006).

One common application of CFA is model comparison. Model comparison is achieved through comparing the fit indices of a constrained model with a less constrained or unconstrained 
hierarchical model. Constraints may be imposed on factor loadings, on the intercepts/thresholds or on error terms.

When comparing models (e.g. across groups), parameter estimation may proceed in three different ways: (1) some parameters are allowed to be freely estimated, in which the researcher allows the free estimation of parameters, such as the factor loadings, factor correlations, and unique variance); (2) some parameters are fixed to a value, where the researcher specifies a value for certain parameters, for example, equaling factor cross-loadings to zero, or equaling one factor loading to one for the purpose of removing scale indeterminacy; and (3) some parameters are constrained, in which the researcher imposes a condition on the parameters without specifying a value, for example equality of factor loadings across groups.

As stated above, the latent variable in factor analysis is unobserved and hence lacks a scale. The problem of scale indeterminacy is overcome in CFA in two ways. In the first method, the researcher fixes the metric of the latent variable to be the same as one of the loading indicators, which is called the marker or the reference indicator. This method is used in tests of measurement invariance across groups and evaluation of scale reliability. In the second method, the variance of the latent variable is fixed to a specific value, which is commonly 1.00 .

\subsubsection{Parameters of a CFA model}

A basic CFA model has factor loadings, unique variances, and factor variances. Factor loadings are akin to regression slopes for predicting the indicators' values from the latent factor. In other words, factor loadings are estimates of the direct effects of factors on indicators. Standardized factor loadings (on one factor) are the estimated correlations between an indicator and its factor, and which when squared show the amount of variance explained (an RSQ > .50 is desirable). When we have multiple latent variables, standardized factor loadings are beta weights 
that control for correlated factors and since they are not correlations, they cannot be squared to produce explained variance.

The unique variance is the variance in the indicator that is not explained by the latent factors under study (akin to the residual in regression analysis). The factor variance indicates the extent to which sample participants' relative standing on the latent continuum varies.

A CFA model may also include other parameters in line with the hypotheses of the researchers or the theory. For instance, two indicators may covary for reasons other than the shared influence of the latent factor. In this case, error covariance is another parameter which may be estimated. In addition, if the CFA model contains more than one latent variable, one may wish to estimate factor covariance to estimate the relationship between latent dimensions. If a CFA model is designed to incorporate mean structures in addition to the variance-covariance matrix, the researcher may wish to estimate indicator intercepts and the latent factor means (which similar to an ANOVA is used to compare groups on a certain trait). With the addition of the indicator intercept to the model, the CFA equation changes to:

$$
X_{i}=\tau_{i}+\Lambda_{i} \xi+\delta_{i}
$$

As Brown (2006, p. 72) stated, "The objective of CFA is to obtain estimates for each parameter of the measurement model (i.e., factor loadings, factor variances and covariances, indicator error variances and possibly error covariances) that produce a predicted variancecovariance matrix (denoted $\boldsymbol{\Sigma}$ ) that represents the sample variance-covariance matrix (denoted $\mathbf{S}$ ) as closely as possible." In other words, the purpose is to see if the model fits the data. 
As with any statistical modeling approach, various fit indices are introduced to verify the accuracy of the reproduced covariance matrix. Therefore, assessing model fit plays an important role in concluding that the hypothesized model is appropriate.

\subsubsection{Parameter Estimation Methods in CFA}

Estimation of parameters in a CFA analysis depends on the nature of the data, statistical assumptions, and completeness of the raw data. There are several parameter estimation methods used in CFA/SEM analysis. The most common methods are maximum likelihood estimation (MLE), weighted least squares (WLS), robust weighted least squares, and robust maximum likelihood. The following sections briefly describe each of these common parameter estimation methods in the CFA/SEM analysis.

\subsubsection{Maximum Likelihood Estimation}

Maximum likelihood estimation (MLE) relies on the likelihood function and principle. The likelihood function is a data reduction device through which desired parameters are obtained given a sample of observations.

To define the likelihood function formally, if we let $f\left(x \mid \theta_{1}, \ldots, \theta_{k}\right)$ denote the joint probability density function (pdf) or the probability mass function (pmf) of the sample $\mathrm{X}=\left(\mathrm{X}_{1}\right.$, $\left.\ldots, X_{n}\right)$, then given that $X=x$ is observed, the function of $\theta$ defined below is the likelihood function:

$$
L(\theta \mid x)=L\left(\theta_{1}, \ldots, \theta_{k} \mid x_{1}, \ldots, x_{n}\right)=\prod_{i=1}^{n} f\left(x_{i} \mid \theta_{1}, \ldots, \theta_{k}\right)
$$

The difference between the likelihood function and the pdf/pmf is that while in $\mathrm{pdf} / \mathrm{pmf} \mathrm{x}$ is variable and $\theta$ fixed, in likelihood function $\theta$ is variable and $\mathrm{x}$ is fixed. 
The likelihood function finds several estimates of the $\theta$ and chooses the one that best reproduces the pdf or pmf of the sample. The best estimate of $\theta$ is one that maximizes the likelihood function (i.e. the value of $\theta$ at a global maximum). If the likelihood function is differentiable in $\theta_{\mathrm{i}}$ then the plausible candidates for the maximum likelihood estimate are the values of $\left(\theta_{1}, \ldots, \theta_{k}\right)$ that solve

$$
\frac{\partial}{\partial \theta_{i}} L(\theta \mid x)=0, \quad i=1, \ldots, k
$$

Points found in the first (partial) derivative could be local or global minima, local or global maxima, or inflection points (Casella \& Berger, 2002).

According to statistical theory and simulation studies, ML based on product-moment correlation or covariance matrix when using ordinal variables does not perform well. The problems that specifically arise include inflated $\chi^{2}$ test statistic, underestimated parameters, and underestimated standard errors (Flora \& Curran, 2004). The primary reason for this problem is that the covariance structure hypotheses $(S=\Sigma)$ is intended for latent response variables and does not hold for observed ordinal variables (Bollen, 1989). Therefore, polychoric correlations may be used instead of the product-moment correlation. Nevertheless, replacing polychoric correlation coefficients in the correlation matrix for ML will not produce correct test statistics and standard errors (Babakus et al., 1987; Dolan, 1994; Rigdon \& Ferguson, 1991).

\subsubsection{Weighted Least Squares}

MLE should not be used for parameter estimation when there is at least one indicator in the instrument which is categorical. Use of MLE for categorical data has potential consequences, such as attenuated estimates of correlations, presence of pseudo-factors that are produced by item 
difficulties or extremeness, and incorrect test statistics and standard errors (Brown, 2006). When the indicators are categorical, the $\mathbf{S}$ matrix consists of tetrachoric (for binary indicators) and polychoric (for polytomous indicators) correlation coefficients than covariance coefficients.

In the presence of categorical data, parameter estimation methods include weighted least squares (WLS), robust weighted least squares (WLSMV), and unweighted least squares (ULS). The most common estimation methods for categorical indicators in latent variable modeling are WLS (originally developed for nonnormal data) and WLSMV.

WLS is very similar to general least squares (GLS) method. GLS minimizes the discrepancy between the observed and predicted covariance matrices. However, in GLS, the weight matrix is usually the inverse of the observed covariance matrix (S) while in WLS the weight matrix is based on the estimates of the variances and covariances of each element of $S$ and the multivariate fourth-moment to adjust for violations in multivariate normality.

WLS takes into account the asymptotic variances and covariances of polychoric correlations through a weight matrix $\mathrm{W}$ which is positive-definite. WLS applies the fitting function:

$$
F_{W L S}=[s-\sigma(\theta)]^{T} W^{-1}[s-\sigma(\theta)]
$$

where $\mathrm{s}$ is a vector of polychoric correlations, $\sigma(\theta)$ is the model-implied vector of population elements in $\Sigma(\theta)$, and $\mathrm{W}$ is a positive-definite weight matrix. $F_{W L S}$ is also called asymptotically distribution free (ADF) estimator because no particular distribution is specified for the function. WLS was originally developed to ameliorate the effect of nonnormality in the data and was not intended for categorical indicators. However, B. Muthen $(1983,1984)$ generalized WLS to a 
combination of dichotomous, ordered categorical, and continuous variables through the use of polychoric correlations among ordinal observed variables and then fitting an SEM model with WLS estimation, providing asymptotically unbiased estimates and correct chi-square test of fit with dichotomous or ordinal observed variables.

Although WLS can produce asymptotically unbiased estimates, there are some drawbacks with WLS even with polychoric bivariate correlations. One grave problem with WLS is that as the number of indicators increases, the $\mathrm{W}$ matrix increases fast and becomes nonpositive definite and hence noninverible. Research has shown that WLS does not perform accurately in small and moderate sample sizes (Flora \& Curran, 2004). An alternative to WLS is robust WLS.

In robust WLS (B. Muthen, du Toit \& Spisic, 1997), the W matrix in equation 5 is replaced with diagonal matrix $\mathrm{V}$ which includes the asymptotic variances of the thresholds and polychoric correlation estimates. After obtaining the vector of parameter estimates, standard errors are estimated using a robust asymptotic covariance matrix which is calculated through the W matrix. Next, a robust goodness-of-fit test is calculated which is based on the mean- and variance-adjusted chi-square test statistic (hence WLSMV) using the full weight W matrix without inversion. In robust WLS, the value for model degrees of freedom is estimated from empirical data rather than from the specification of the model. Because WLSMV uses the full W matrix, the sample size required is less restrictive than that of the WLS (samples as small is 200; Brown, 2006).

\subsection{Model Fit Indices in CFA}

There are basically three sample-based approaches to assessing model fit in CFA. The first approach is to examine the residual matrix which contains the predicted covariance 
coefficients minus the observed covariance coefficients. Although the residual matrix can provide useful information regarding model fit, it can be difficult to interpret for a moderate number of indicators.

The second approach is to conduct a hypothesis test using, for example, the $\chi^{2}$ test statistic. The $\chi^{2}$ test, or its asymptotic equivalents in the forms of Lagrange multiplier or the Wald test, are used to assess the appropriateness of model parameter constraints in the SEM model (Raykov \& Marcoulides, 2006). Historically, the $\chi^{2}$ test has received considerable attention because it is used as a distribution in several other fit index formulations and because of its inferential property (Raykov \& Marcoulides, 2006).

The disadvantage of using the $\chi^{2}$ test statistic is that it tests a meaningless null hypothesis that states the model fits perfectly in the population. In addition, the $\chi^{2}$ value is dependent on sample size and is usually significant for large sample sizes, though, it is a good statistic for nested models (Thompson, 2004; Harrington, 2009). When the sample size increases, the value of the $\chi^{2}$ test statistic increases (as multiplied by $N$-1) while its degrees of freedom remain unchanged (not penalized). The $\chi^{2}$ index is especially biased when the maximum likelihood estimation method is used and when the multivariate normality is inadequate (Thomson, 2004). Moreover, it is susceptible to large correlations among the observed variables and the unique variance (Kline, 2011). A more useful approach based on hypothesis testing is to test a null hypothesis where the model is not expected to fit perfectly, but good-enough. This approach relies on constructing a confidence interval for a fit index (e.g., root mean square error of approximation, RMSEA) and determining if the confidence interval is below or above a certain cutoff value. 
The third approach relies on summarizing the model fit using an index and comparing the fit index to a predetermined cutoff value. If the fit index exceeds a particular value, then the researcher concludes that the model fits well (but not necessarily perfectly) in the population. This approach often requires the researcher to use multiple fit indices to provide a broad assessment of the model fit. Gerbing and Anderson (1992) state that an ideal fit index should have three characteristics: (1) has a scaled range between 0 and 1, (2) is independent of sample size, and (3) has known distributional properties to assist interpretation. In addition, Fan, Thompson and Wang (1999) assert that an ideal fit index is expected to have (1) as little systematic bias as possible, and (2) as little random variation as possible. A model may have poor fit due to a number of causes, including specifying too few or too many factors, choosing inappropriate indicators, defining an incorrect pattern of indicator-factor loadings or misspecification of error theory (Harrington, 2009).

Three common fit indices that will also be used in this study are the root mean square error of approximation (RMSEA), comparative fit index (CFI), and Tucker and Lewis nonnormed fit index (TLI).

Root mean square error of approximation (RMSEA). The RMSEA is a parsimonyadjusted measure of model discrepancy in the population and is computed as,

$$
\varepsilon=\sqrt{\frac{\delta}{d f(N-1)}}
$$

where $\delta$ is the non-centrality parameter (measure of discrepancy between hypothesized and sample covariance matrices up to the expected value of the model $\chi^{2}$ or model degrees of 
freedom; see Cumming and Finch, 2001; Kline, 2011), $d f$ is the degree of freedom and $N$ is the sample size. $\delta$ is defined as follows:

$$
\delta=\max \left(\chi^{2}-d f, 0\right)
$$

RMSEA penalizes free parameters through dividing them by $d f$. It also rewards a large sample size because $N$ is in the denominator. RMSEA values less than .06 support reasonable model fit (Hu and Bentler, 1999; Brown, 2006). Close fit (CFit) is a statistic that tests the probability that RMSEA is below the chosen threshold (Brown, 2006). The close fit (CFit) test was developed by Browne and Cudek (1993) as a significance test for RMSEA. Specifically, CFit tests if the probability of an RMSEA .05 is statistically significant or not. Nonsignificant values are supportive of the acceptable model fit.

RMSEA is sensitive to model complexity in small sample sizes (Breivik \& Olsson, 2001) but insensitive to sample size (Harrington, 2009). As the sample size and degrees of freedom increase, the value of RMSEA decreases. However, RMSEA is not biased for larger sample sizes or greater degrees of freedom because as the sample size increases the correction for parsimony decreases (Mulaik, 2009). RMSEA is based on the non-central $\chi^{2}$ distribution. Failure to meet this distribution may result in incorrect confidence interval bounds (Kline, 2011). RMSEA is limited in three ways (Kline, 2011): (1) interpretation of the RMSEA point and interval estimations depends on meeting of the non-centrality $\chi^{2}$ distribution; (2) RMSEA is dependent on multivariate normality; and (3) in complex but small models, RMSEA produces inaccurate results. 
Comparative fit index. Bentler's Comparative Fit Index (CFI) is also a model discrepancy fit index based on the non-centrality measure that compares the fit of the model with a baseline (independence) model. CFI is derived as:

$$
C F I=1-\frac{\hat{\delta}_{M}}{\hat{\delta}_{B}}
$$

The $\delta$ 's are calculated for the researcher's model (M) and the baseline or null model (B). The baseline model is a null or independence model in which the covariances among all input indicators are fixed to zero. A CFI > .95 is commonly used to indicate good fit (Hu and Bentler, 1999; Brown, 2006). CFI is the scaled form of McDonald and Marsh (1990) relative noncentrality index (NRI). Incremental fit indices like CFI have been criticized for relying on the independence model because the assumption of zero covariances among indicators is unrealistic (Kline, 2011).

Tucker Lewis index. Tucker Lewis Index (TLI), also known as non-normed fit index (NNFI) due to its bounds that can exceed the 0-1 interval, is another model discrepancy fit index which compares the fit between the target model and the baseline model:

$$
T L I=\frac{\frac{\chi_{B}^{2}}{d f_{B}}-\frac{\chi_{M}^{2}}{d f_{M}}}{\frac{\chi_{B}^{2}}{d f_{B}}-1}
$$

TLI corrects the model for parsimony by penalizing the free parameters via dividing by degrees of freedom. Like CFI, TLI is a function of the average correlation among the indicators. 
A TLI > .95 is commonly used to indicate good fit (Hu \& Bentler, 1999; Brown, 2006). Because TLI and CFI are highly correlated, it is common to report one of them in research reports.

There have been several studies that have examined fit indices in CFA and how they behave under various conditions. Comparative fit indices such as CFI have been shown in previous studies to be sensitive to sample size with different degrees (Fan, Thompson \& Wang, 1999). Kenny (2012), for instance, found that CFI and TLI are artificially increased when the correlations between the indicators are high because the baseline model (where all variables are uncorrelated) has a large discrepancy with the empirical correlation matrix in the case of high correlations between the indicators within the clusters, giving rise to a much larger chi-square statistic than the implied correlation matrix, which affects the fractions in CFI and TLI, inflating the quotient.

Fan, Thompson, and Wang (1999) conducted a simulation study on the effect of sample size, estimation procedure, and model misspecification on a variety of fit indices. The RMSEA tended to suggest better fit when using generalized least squares estimation compared to the ML method. Under different sampling bias, the CFI showed relatively slight negative sampling bias. They also found that RMSEA was most susceptible to variation under different model misspecifications. As for sample size, RMSEA, CFI and TLI were least sensitive to sample size variation. The authors concluded that the comparative fit indices are less stable across estimation methods than absolute fit indices, especially for severely misspecified models.

In their study on the effect of the number of variables on SEM fit indices, Kenny and McCoach (2003) found that RMSEA improved with increasing the number of indicators while CFI and TLI generally, though not always, tended to deteriorate with increasing the number of indicators in the model. Ding, Velicer, and Harlow (1995) found that in a perfectly specified 
model, the CFI and the TLI deteriorated as the number of indicators increased under ML method, though with large sample sizes they were close to 1 . In a similar study, Marsh, Hau, Balla, and Grayson (1988) found that the chi-square tended to penalize models with a large number of indicators. Anderson and Gerbing (1984) also reached a similar conclusion in their study, where the fit indices deteriorated as the number of indicators per factor increased (in particular, the TLI).

Kenny and McCoach (2003) concluded that in perfectly specified models, a deterioration in fit indices shows that there is a slight amount of bias in several commonly used measures of fit (p. 338). In a study under misspecified models, Breivik and Olsson (2001) found that the RMSEA value deteriorated as the number of indicators increased in the model and the CFI was variably, and to a lesser degree, affected. In a similar study, Chau and Hocevar (1995) found that in misspecified models, both the CFI and TLI significantly decreased as the number of observed variables increased using the ML method.

Kenny and McCoach (2003) conducted a study where they varied the sample size to test the effect on fit indices. They found that both the CFI and the TLI decreased as the number of indicators increased in the model, while RMSEA tended to improve, particularly in larger sample sizes. They found that RMSEA improved as the number of indicators in the model increased. As for the CFI, it decreased as the number of indicators increased (in the minor-factor and twofactor models) while for the method-error source, the CFI increased slightly. Because CFI and TLI are similar in derivation, the TLI also showed similar pattern to CFI in their study. The authors concluded that the effect of the number of variables on fit indices is a function of a number of factors, including the type of the model, the type and degree of misspecification, distributions of the variables, the sample size, the estimation method used, and the measure of fit. 
In misspecified models, the TLI and the CFI both declined and improved depending on the type of misspecification as more indicators were added, while RMSEA tended to improve. In correctly specified models, both TLI and the CFI deteriorated as more variables were added to the model, while RMSEA tended to improve. Therefore, TLI and CFI do not function well (in either direction) when more indicators are added to the model. The authors recommend inspecting simultaneously RMSEA and CFI/TLI in models with large number of indicators: if TLI and CFI seem lower than expected but RMSEA is a bit better, then there is no real cause for concern. However, if the three of them show poor fit, then the misfit is serious.

Despite several studies comparing the statistical tenability of fit indices, one needs to consider other factors in the choice of appropriate fit indices. As Brown (2006, p. 82) aptly poses: What fit indices should be used in what contexts? And what cutoff values should be used to indicate acceptable fit?

\subsection{Multiple-group Confirmatory Factor Analysis}

Confirmatory factor analysis (CFA) has been widely used in assessing measurement invariance (MI) across manifest groups. Because in measurement invariance analyses at least two groups are involved, the common CFA model is extended for the MI testing purposes by extending it over multiple groups. One popular CFA model for assessing MI is MG-CFA. For example, Schmitt and Kuljanin (2008) reported that MG-CFA studies in establishing scalar invariance have increased in the past two decades from $12 \%$ to $54 \%$ of the reviewed studies and that from 88 studies they reviewed, 75 (85\%) had used CFA for investigating different levels of invariance. 
In the common-factor CFA framework, to establish measurement invariance, different (nested) tests can be conducted, each testing one aspect of the measurement model, which is based on the following two equations:

$$
\begin{aligned}
& X_{k}^{g}=\tau_{k}^{g}+\Lambda_{k}^{g} \xi^{g}+\delta_{k}^{g} \\
& \Sigma^{g}=\Lambda_{X}^{g} \Phi^{g} \Lambda_{X}^{g^{\prime}}+\Theta_{\delta}^{g}
\end{aligned}
$$

In equations (10) and (11), $X_{k}^{g}$ refers to the vector of item responses, $\tau_{k}^{g}$ refers to the vector of regression intercepts, $\Lambda_{k}^{g}$ refers to the matrix of regression slopes, $\xi^{g}$ is the common factor, $\delta_{k}^{g}$ refers to the vector of unique factors, $\Sigma^{g}$ refers to the variance-covariance matrix among the $k$ items in each group, $\Lambda_{X}^{g}$ refers to the matrix of factor loadings, $\Phi^{g}$ refers to the variance-covariance matrix for inter-factor relationship, and $\Theta_{\delta}^{g}$ is the diagonal matrix of unique variances.

Based on the number of parameters being estimated, eight different equality tests can be performed, five of which can be called tests of aspects of measurement invariance (relationships between measured variables and latent factors; Category 1 tests as classified by Little, 1997) and the last three of which test aspects of structural invariance (tests about latent factors themselves; Category 2 tests as classified by Little, 1997) (Byrne, Shavelson, \& Muthen, 1989; Schmitt \& Kuljanin, 2008). The implementation of these tests depends on the goal of the study (Schmitt \& Kuljanin, 2008). Because in this study we are concerned only with measurement invariance, only the first five tests are elaborated below:

(1) $\Sigma^{g}=\Sigma^{g \prime}$ In this test, the researcher attempts to establish the equality of the variancecovariance matrices derived from the different subpopulations. Failure to reject the null 
hypothesis that $\Sigma^{g}=\Sigma^{g^{\prime}}$ through $\chi^{2}$ or other fit statistics shows the equality of measurement equivalence and allows the researcher to dispense with further MI tests. However, rejection of the null hypothesis shows that a source of variance should exist in the measurement model (Schaubroeck \& Green, 1989; Schmitt, 1982; Vandenberg \& Self, 1993) and further and more restrictive tests are needed to detect the source or cause of invariance (Byrne, 1989).

(2) $\xi^{g}=\xi^{g \prime}$ In this test, known as configural invariance, the researcher attempts to establish the equality of the factor structure across the groups. This is the test of the null hypothesis that the a priori pattern of free and fixed factor loadings is equal across groups. Failure to reject the null hypothesis implies that the factor structure or dimensionality between the groups is comparable and allows us to continue performing subsequent tests of MI. The rejection of the null hypotheses, however, means that the two groups are employing different factor structures, and hence further tests of MI are not warranted because the two latent factors and their properties are not comparable. In the absence of configural invariance, comparison of groups over a given construct would accrue inaccurate interpretations (Millsap \& Olivera, 2012).

(3) $\Lambda_{k}^{g}=\Lambda_{k}^{g \prime}$ In this test, also known as metric invariance, the researcher aims to establish the equality of the factor loadings across groups. In metric invariance test, the factor loadings of similar items are constrained to be equal across groups. Factor loadings are in fact the regression slopes from observed variables to their corresponding latent factors. In the metric invariance analysis, one indicator factor loading is fixed to 1 as a referent indicator and regarded invariant. In a single-factor model, and under certain conditions, modification indices may be used to identify the referent item (Yoon \& Millsap, 2007). This form of measurement invariance is called strong invariance. Some authors (e.g. Bollen, 1989; Millsap \& Hartog, 1988) have proposed that once the null hypothesis is rejected, further tests of measurement invariance are 
not warranted; others (e.g. Byrne et al., 1989; Steenkamp \& Baumgartner, 1998) have contended that partial measurement invariance (i.e., a subset of the factor loadings are invariant) is permissible in case of the rejection of the null hypothesis.

(4) $\tau_{k}^{g}=\tau_{k}^{g \prime}$ In this test, referred to as scalar invariance, we test if the vector of item intercepts or thresholds are invariant across groups. This test has been interpreted as systematic response bias, especially in response rating. If scalar invariance is established, any differences in observed scores between groups can unambiguously be attributed to their differential constructs (Millsap \& Olivera, 2012). This test also allows the establishment of the equality of factor means across groups (Schmitt, \& Kuljanin, 2008).

(5) $\Theta_{\delta}^{g}=\Theta_{\delta}^{g \prime}$ This is a test of unique variances equality across groups. Although the $\Theta$ matrix is often diagonal, covariances may exist among items to control for correlated measurement errors due to, for example, local dependencies for items that share a common stimulus. To perform this test, the item uniqueness values (deltas) are constrained to be equal across groups. For the item residuals covariances, the covariances between adjacent items may be constrained to test the plausibility of first-order auto-correlated structure on the uniqueness (Willet \& Sayer, 1994). If the latent factor variances are equal, test of residual invariance is also known as the test of the equality of the reliability of the observed variables (Vandenberg \& Lance, 2000). Invariance of the residuals is often called strict invariance (Meredith, 1993). Some researchers believe that attaining residual invariance is not necessary to test equality of factor structure or latent means (Widaman \& Reise, 1997; Byrne \& Stewart, 2006).

Although there are several tests of invariance that can be performed, we are typically interested in three types of measurement invariance: configural, metric, and scalar (Vandenberg 
\& Lance, 2000). Therefore, in this study, I will investigate the three aforementioned types of measurement invariance.

When invariance fails at one of the foregoing steps, the researcher may choose a subset of the parameters, such as some of factor loadings, some of the item intercepts, to establish measurement invariance. Because the researcher is not using all the parameters, this is called partial measurement invariance analysis. In other words, a partially invariant model is one in which a subset of the model parameters is invariant (Byrne, Shavelson, \& Muthen, 1989). Partial MI is based on the reasoning that measurement invariance may hold for part of the measured variables across populations and relaxing invariance constraints where they do not hold controls for partial measurement variance (Vandenberg \& Lance, 2000).

Some researchers (e.g. Bagozzi \& Edwards, 1998 and Reise, Widaman \& Pugh, 1993) discuss tests of partial invariance in the metric equivalence context while Steenkamp and Baumgartner (1998) recommend partial measurement invariance testing at all steps of measurement invariance analysis. In tests of partial invariance, researchers examine the modification indices or the relative sizes of parameters across groups and allow parameters to be free until the remainder of the parameters is invariant across groups. A potential problem in partial invariance is that variability in subgroup parameters across indicators may be contradictory, that is, higher in one comparison for one group and lower in comparison for that group for another parameter (Schmitt \& Kuljanin, 2008). Partial invariance is best achieved when the researcher is guided by some prior experience or theory (Millsap \& Olivera, 2012).

\subsection{Model fit criteria in MG-CFA}

The performance of different goodness of fit indices in MI tests is an important yet unanswered question (Chen, 2007). Because MI in this study is based on CFA, several fit indices 
relevant to CFA are recommended for the full analysis of MI. Three model fit analyses are recommended (Vandenberg \& Lance, 2000): overall model fit, model differences fit, and reference indicator selection.

The overall model fit assesses how well the model-implied covariance matrix can represent the empirical covariance matrix obtained from the observed data. A poor model fit shows that the measures were not configured properly in each group, hence precluding further tests of MI. The most common fit indices in CFA literature are Tucker-Lewis index (TLI), also called non-normed fit index (NNFI), relative noncentrality index (RNI), root mean square error of approximation (RMSEA) and the standardized root mean square residual (SRMR). Another widely-used fit indicator is the comparative fit index (CFI).

In the model differences fit assessment, the purpose is to investigate any worsening of fit of the more constrained model compared to a direct previous and less constrained model. In other words, the researcher attempts to see if the more parsimonious model fits the data as well as a more complex model. The most commonly used model difference fit statistic is the $\chi^{2}$ change value $\left(\Delta \chi^{2}\right)$ although some researchers argue that this index should not be relied upon as the sole indicator of model fit (Brannick, 1995; Cheung \& Resnvold, 1999; Kelloway, 1995).

In partial metric invariance, the researcher is interested in identifying and then freeing the offending invariance constraint (i.e., the item or items that calibrated to the underlying construct differently in a group) that caused the model to fit poorly. The decision to free the constraint is determined by assessing the fit of the new partial-metric invariant model and the baseline or the configural model. If the new model has a fit as good as the baseline model, then the researcher has identified the reference indicator items. 
As stated above, MG-CFA is an extension of the CFA model fitting procedure where the invariance of estimated parameters of two nested models across multiple groups is tested. For example, if the researcher attempts to test metric invariance, the decision is to compare the fit of an unconstrained model with no restrictions on model parameters with a constrained model in which all factor loadings are constrained to be equal across all groups. If the imposition of the constraints substantially worsens the fit statistic, then the constraint is inappropriate and should be removed because the constrained parameters are noninvariant (Cheung \& Rensvold, 2002).

The amount of difference between the two models is evaluated using different fit indices, the most frequently used of which is the likelihood ratio (LR) test which measures the differences in $\chi^{2}$ between the two models (Cheung \& Rensvold, 2002). The $\chi^{2}$ difference is calculated as:

$$
\Delta \chi^{2} \therefore \chi_{c}^{2}-\chi_{u c}^{2}
$$

The $d f$ for $\Delta \chi^{2}$ equals the difference in the $d f$ for the respective models (i.e., $d f=d f_{c}-d f_{u c}$ ). An unappealing feature of the $\chi^{2}$ difference test is that it is often statistically significant because the hypothesis being tested is technically false and we often use very large sample sizes that provide sufficient power to reject the false null hypothesis. Hence, the change in $\chi^{2}$ statistics may not be a useful measure of non-negligible measurement invariance (Brannick, 1995; Kelloway, 1995; Cheung \& Rensvold, 2002). Therefore, researchers are seeking fit criteria which are independent of model characteristics.

To address the limitation of the change in $\chi^{2}$ test, Cheung and Rensvold (2002) proposed examining the change in goodness-of-fit indices (GFI) instead. The authors evaluated the 
performance of 20 GFIs used in establishing measurement invariance. The GFIs included the chi-square $\left(\chi^{2}\right)$, normed chi-square $\left(\chi^{2} / \mathrm{df}\right)$, the noncentrality parameter (NCP), root mean standard error of approximation (RMSEA), Akaike's information criterion, Browne and Cudeck's Criterion, Expected Cross-Validation Index, Normed Fit Index (NFI), Relative Fit Index (RFI), Incremental Fit Index (IFI), Tucker-Lewis fit Index (TLI), Comparative Fit Index (CFI), Relative Non-centrality Index (RNI), parsimony adjusted NFI, parsimonious CFI, Gamma hat, rescaled Akaike's Information Criterion, cross-validation index, McDonald's Non-Centrality Index and Hoelter's critical $N$.

In their study, Cheung and Rensvold (2002) simulated data for six conditions: number of factors ( 2 or 3 factors), factor variance (.36 or .81 ), correlations between factors ( .3 or .5 ), number of indicators per factor $(3,4$, or 5$)$, factor loadings (two patterns for each number of indicators), and sample sizes per group (150 or 300). Maximum likelihood was used in all 48 models as estimation method. To further reflect real situations, factor reliability was set at .80. In addition, model fit values were chosen not to be perfect, so the covariance matrices were generated to produce CFI, TLI and RMSEA fit values of $.97, .96$, and .057 , respectively. Based on these simulation configurations, eight tests of measurement invariance were performed to evaluate the sensitivity of the selected GFI's in each model.

A six-way ANOVA was performed to study the simple effect and interaction effect of the six simulation factors on the $20 \mathrm{GFI}$ indicators. At the configural MI test, the ANOVA results showed that factor loadings, factor variances, and factor correlations had no effect on any of the GFI indicators. However, the number of items, number of factors, and the item-by-factor interactions had a significant effect on the values of GFI indices. Therefore, it appeared that model complexity affected the performance of GFI indices. Although most GFI indicators 
accommodate for model complexity, in this simulation only RMSEA managed to do so.

Therefore, for different factor complexity, different cut-off values should be used.

To select the best performing GFIs, Cheung and Rensvold (2002) in their study set three criteria and selected those $\Delta$ GFIs that met these selection criteria. The first criterion was that a $\Delta$ GFI should not correlate significantly with its corresponding GFI. By this criterion, NCP, IFI, CFI, RNI, Gamma hat, McDonald's NCI, and critical N were candidate GFIs. A second criterion was that a $\Delta$ GFI should not be affected by model complexity. $\Delta \mathrm{IFI}, \Delta \mathrm{CFI}, \Delta \mathrm{RNI}, \Delta \mathrm{Gamma}$ hat, and $\triangle \mathrm{McDonald}$ 's NCI met this criterion. A third criterion was that a $\triangle \mathrm{GFI}$ should not correlate with another $\Delta$ GFI. CFI, $\Delta$ CFI, Gamma hat, $\Delta$ Gamma hat, McDonald's NCI, and $\Delta$ McDonald's NCI met this criterion.

In the final selection of the best performing GFIs, Cheung and Rensvold used the 1st or 99th percentile values of the examined $\Delta$ GFI's as the critical values for rejecting the null hypothesis of invariance with an alpha level of 0.01 and assuming multivariate normality. In conclusion, Cheung and Rensvold (2002) reported that $\Delta \mathrm{CFI}, \Delta$ Gamma hat, and $\Delta$ McDonald's NCI were not affected significantly by model complexity and sample size. They proceeded to propose the following general cutoff values of $\Delta \mathrm{CFI} \leq-0.01, \Delta \mathrm{Gamma}$ hat $\leq-.001$ and $\Delta$ McDonald's $\mathrm{NCI} \leq-.02$. These values are obtained by averaging and rounding $\Delta \mathrm{GFI}$ across different MI tests. The exact values of the obtained $\Delta$ GFI at each MI test level are presented in Table 1.

Meade, Johnson, and Braddy (2008) replicated Cheung and Rensvold's (2002) study with the additional condition of introducing varying degrees of lack of invariance (LOI). In other words, Meade et al. (2008) attempted to investigate the power of alternative MI fit indices (AFI) when degrees of MI at factor structure, factor loadings, and item intercepts were present (unlike 
Cheung and Rensvold, who averaged their proposed cut-off values across all MI levels). At configural LOI, noninvariance ranged from 0 to all nonreference indicator items that loaded onto an unmodeled factor (i.e. crossloaded); at factor loading invariance, LOI was imposed on $25 \%$ of items with factor loadings differing from 0 to 0.40 in increments of .02; and at scalar invariance level, LOI was imposed on $25 \%$ of items with intercepts differing from 0 to .30 in increments of .02. This study was performed in three phases.

In the first phase, different degrees of MI at configural, metric and scalar levels were introduced and the performance of $\triangle$ AFIs were evaluated with regard to their sensitivity to LOI, sample size, model complexity, fit of the baseline model and correlation with other $\Delta$ AFIs. In this phase, items exhibiting differential item functioning (DIF) were introduced in one group in an instrument with four correlated factors. There were 16 items evenly and error-free loading on the four factors. The intercepts in group 1 were set at zero and error terms set to equal. In addition, slight model misfit was introduced by cross-loading some items on other three factors from a normal distribution. Then the performance of $\Delta$ AFIs was examined for two conditions: number of items per factor and the number of factors (creating four conditions: two factor conditions (2 or 4$)$ and three item conditions (4, 8, and 32).

The study variables manipulated in this phase included the number of factors, the number of indicators, the level of LOI and the sample size. Sample sizes included 100, 200, 400, 800, 1600, and 6400 to reflect the common Ns in practice. Therefore, four models with six sample sizes were included in the first phase of the study. The sample size, number of factors, and the number of indicators were the same in the configural, metric and scalar MI tests. The $\Delta \mathrm{AFIs}$ investigated in this phase of the study, chosen for their lack of correlation with the baseline 
model fit index, included $\Delta \mathrm{CFI}, \Delta \mathrm{Gamma}$ hat, $\Delta \mathrm{Mc}, \Delta \mathrm{NCP}, \Delta \mathrm{IFI}, \Delta \mathrm{RNI}, \Delta$ critical $\mathrm{N}$ and $\triangle$ RMSEA.

The suitability of each $\Delta$ AFI was assessed using the $p$ value and the $\omega^{2}$ as the effect size in an $n$-way ANOVA. $\triangle$ AFIs were considered optimal if they showed large $\omega^{2}$ for the level of LOI variable and small $\omega^{2}$ for other study variables. For the configural invariance investigation, the authors focused on the performance of $\chi^{2}$, CFI, Mc, RMSEA, and SRMR. The results showed that when there were one or two DIF items, all AFI's rarely exceeded the recommended cut-off values in the literature (i.e. $\mathrm{CFI}=.95, \mathrm{Mc}=.90, \mathrm{RMSEA}=.06$, and $\mathrm{SRMR}=.08$ ).

When the number of DIF items increased to one-third of the items, all AFIs detected configural LOI but CFI and Mc seemed to perform better than RMSEA or SRMR. SRMR was more strongly affected by sample size and both RMSEA and SRMR were less sensitive to LOI when the number of items was 8 for each of four factors compared with CFI and Mc. Mc proved to show the best performance in configural LOI detection in all conditions (with a mean above .90). The results in the metric and scalar invariance investigations showed that almost all $\Delta \mathrm{AFIs}$ outperformed $\chi^{2}$ in being responsive to an LOI and insensitive to sample size and model complexity, with the exception of the NCP and critical N (because NCP is defined as $\chi^{2}$ minus degrees of freedom, this index and $\chi^{2}$ had similar effect size estimates). $\Delta$ IFI, $\Delta$ RNI, $\Delta$ Gamma hat, and $\Delta$ CFI performed optimally by being both extremely responsive to different levels of LOI and being unresponsive to other data properties. $\Delta \mathrm{Mc}$ also performed well. These results held for both metric and scalar invariance tests. For the effect of model misfit on $\Delta \mathrm{AFI}$, the results showed that $\Delta \mathrm{IFI}, \Delta \mathrm{RNI}, \Delta \mathrm{Gamma}$ hat, and $\Delta \mathrm{CFI}$ all performed quite well, except for the $\Delta \mathrm{Mc}$ and $\triangle \mathrm{RMSEA}$. At this phase, the authors recommended the use of $\triangle \mathrm{CFI}$ and $\triangle \mathrm{Mc}$ as better performers. 
In the second phase of the study, $\triangle \mathrm{AFIs}$ (selected from the first phase, i.e. $\Delta \mathrm{CFI}, \Delta \mathrm{Mc}$, and $\triangle$ RMSEA) were computed for metric and scalar invariance under different model conditions to yield empirical cut-off values. As for the conditions for investigation, the authors chose the number of items and the number of factors under which to investigate the performance of the selected $\triangle$ AFIs (factor loadings $=.7$, factor correlations $=.3$ and sample size $=200$ in all simulations). The number of items varied from 3 to 30 and the number of factors varied from 1 to 10 .

After calculating the metric invariance and scalar invariance $\triangle \mathrm{AFIs}$, the 99 th percentile of the $\triangle$ AFIs were selected as the cutoff values. The effect sizes $\left(\omega^{2}\right)$ were computed using an $n$ way ANOVA with the number of items and factors as variables. All the three selected $\Delta$ AFIs from the first phase, i.e. $\triangle \mathrm{CFI}, \triangle \mathrm{Mc}$, and $\triangle \mathrm{RMSEA}$ performed equally well across all conditions, with robust performance for sensitivity to model complexity (i.e. number of items and factors and their interaction). The cutoff values, which are the standard deviation of the 99th percentiles, for the $\triangle \mathrm{CFI}$ was .0017 for metric invariance, .0018 for scalar invariance, and .0017 across all conditions; for the $\Delta \mathrm{Mc}$ it was .0081 for metric invariance, .0033 for scalar invariance and .0085 for both; for $\triangle$ RMSEA, the cut-off values were .0069 for metric invariance, .0062 for scalar invariance, and .0069 for both.

In the third phase of the study, the authors examined the power and Type I error associated with the $\triangle$ AFIs from phase one and the cutoff values from phase two. Thus, different $\Delta$ AFIs were compared with respect to different study conditions vis-à-vis Cheung and Rensvold's (2002) proposed cutoff values for a general $\Delta$ AFI for use with any set of data. In other words, a general cutoff value for any type of data on the basis of phase two results, cutoff values derived in phase two that closely match the simulation conditions, and cutoff values 
derived by Cheung and Rensvold (2002). Power was defined as the percentage of replications in which the $\triangle$ AFI cut-off value was exceeded.

The results showed that the power of likelihood ratio test was strongly affected by sample size. The power of $\triangle \mathrm{CFI}, \Delta \mathrm{Mc}$, and to a lesser extent, $\triangle \mathrm{RMSEA}$ was quite good for the general cutoff values. For different conditions, $\Delta$ CFI general cutoff score performed quite acceptably. The general cut-off score for $\Delta \mathrm{Mc}$ was less stellar under different conditions unlike its conditionspecific counterpart. For the $\triangle$ RMSEA, the condition-specific power was excellent and the general cut-off value was also quite good for $\mathrm{N}=200$; however, for $\mathrm{N}=800$, more variability was observed. For the metric invariance test, the authors concluded that for $\triangle \mathrm{CFI}$, a cutoff value of .002 was stable across conditions but the cut-off value for $\Delta \mathrm{Mc}$ should be tailored with the given conditions and sample sizes of larger than 400. $\triangle$ RMSEA showed excessive variability. So the authors recommended $\Delta \mathrm{CFI}$ and $\Delta \mathrm{Mc}$ to be used for metric invariance tests. The same results were derived for scalar invariance, with $\Delta \mathrm{CFI}$ cut-off value stable across conditions and the $\Delta \mathrm{Mc}$ appropriate only when adapted to different conditions, and $\triangle$ RMSEA unstable across conditions.

In conclusion, Meade et al. (2008) proposed to use $\Delta \mathrm{CFI}$ and $\Delta \mathrm{Mc}$ for MI tests because they are less sensitive to sample size compared with likelihood ratio tests, they are more sensitive to LOI than $\chi^{2}$, and they are generally nonredundant with other $\Delta$ AFI's.

To assess the sensitivity of goodness of fit indices under different conditions, Chen (2007) conducted two Monte Carlo simulation studies to evaluate the performance of standardized root mean square residual (SRMR), comparative fit index (CFI), root mean square error of approximation (RMSEA), which are widely used in MI evaluations, gamma hat and McDonald's non-centrality index. In the first study, the performance of the foregoing MI goodness-of-fit indices was evaluated under different sampling variation (sampling error) and 
cut-off values were suggested using the $1 \mathrm{st} / 95^{\text {th }}$ and 5 th/99th percentiles of the goodness-of-fit indices when the Type I error of those indices was known. The second study investigated the performance of those goodness-of-fit indices under different degrees of noninvariance. In addition, Type I and Type II errors were examined in the second study.

In the first study, Chen (2007) examined the performance of the goodness-of-fit indices as a function of sampling variability for seven different MI tests: factor loading, intercept, residual variance, factor variance, factor covariance, factor mean, and the variance/covariance and mean structure. The data were generated using the Monte Carlo procedure and the parameters were estimated using maximum likelihood method implemented in Mplus 3.01. After generating the multivariate normal data, a baseline invariance model was fit and it was the configural, loading, or intercept invariance model. The population values were used as the starting values in the baseline invariance model. Finally, a more restricted invariance model was fit to the data and the goodness-of-fit indices and $\chi^{2}$ difference statistics from the more restricted model were compared to those from the baseline model.

In this phase of the study, two population data were simulated for eight or 12 indicators for a single and two-factor model. The sample sizes (for the sampling variability evaluation) were 150, 250, or 500. Five hundred replications were generated for each condition. The results of the first study showed that in factor loadings, intercepts, and residual variance MI tests, SRMR was more sensitive to sampling variation in factor loadings than in intercepts and residual variances while CFI, RMSEA, Gamma-hat and Mc were equally sensitive to all three levels of sampling variation. For instance, in $\mathrm{N}=300$ and indicators $=8$ condition, the mean values of SRMR were $.021, .003$, and .004 and the 95 th percentiles were $.0368, .0058$, and .0102 for MI tests of factor loadings, intercepts and residual variances, respectively; for CFI, the means were 
$.000, .000$, and .000 and the 5 th percentiles were $-.0062,-.0053$, and -.0058; for gamma hat, the means were $.000, .000$, and .000 , and the 5th percentiles were -.0062, -.0063, and -.0064; for Mc, the means were $.000, .000$, and .000 , and the 5th percentiles were $-.0123,-.0126$, and -.0127 for MI tests of factor loadings, intercepts, and residual variances, respectively.

For the MI in factor variance, covariance, and mean, SRMR proved to be most sensitive to random variation in factor variance, moderately sensitive to random variation in covariance, and least sensitive in latent mean, while CFI, RMSEA, Gamma hat and Mc were equally sensitive to all three levels of sampling variation. As for the impact of the number of indicators (eight vs. 12 indicators), the results showed intangible impacts on changes in goodness-of-fit indices. For example, for CFI, the means were the same in both the 8- and 12-indicator conditions while the 95 th percentiles changed slightly (-.0045 vs. $-.0062,-.0043$ vs. -.0053, and .0050 vs. -.0058, in 8- and 12-indicator conditions, respectively). In addition, the results of this phase of the study showed that as sample size increased, there was less variation in fit indices.

Chen (2007) concluded that in small sample sizes, particularly when relying on SRMR, it would be easier to commit Type I errors. Based on the results of the different simulations under different sampling variations, Chen (2007) in this study used the average values of the 1st/95th or 5th/99th percentiles to propose the following cut-off values for different goodness-of-fit indices: for CFI, RMSEA, Gamma hat, and Mc, similar values were suggested for all three levels of invariance tests: a change of $\geq .005$ or -.010 (rounded) for CFI, a change of $\geq .010$ or .015 for RMSEA, a change of $\leq .005$ or -.008 for Gamma hat, and a change of $\leq .010$ or -.015 for Mc, as CFI, RMSEA, Gamma hat, and Mc are equally sensitive to these levels of invariance. For SRMR, different values were recommended for different levels of invariance tests: when testing loading invariance, a change of $\geq .025$ or .030 is proposed; when testing invariance at the 
intercept and residual variance levels, a change of $\geq .005$ or .010 is proposed as SRMR is more sensitive to loading invariance than to the other two levels of invariance.

In the second part of the study, Chen (2007) introduced different degrees of noninvariance to evaluate the performance of the goodness-of-fit indices for the factor loadings, intercepts and residual variances MI tests. Four factors were manipulated to evaluate the performance of the goodness-of-fit indices: factor complexity (i.e. number of indicators per factor), pattern of noninvariance (uniform vs. mixed), sample size, and the ratio of sample size. Similar to the first part, the number of groups was two, the number of indicators was either eight or 12 and the proportions of invariance were $0 \%, 25 \%, 50 \%, 75 \%$ and $100 \%$; in the latter case, only random variation was the condition. The ratios of sample sizes were 1:1,2:1, or 4:1 with sample sizes of 300, 500, or 1000. Replications with Heywood cases were eliminated. A $2 \times 4 \times$ $3 \times 3 \times 2$ ANOVA was conducted on each fit index to test invariance of factor loadings, intercepts, and residuals.

The results showed that the interaction of pattern of variance and proportion of variance was statistically significant at the factor loadings and intercepts level, but not at the variance residuals level. Pattern of invariance affected all fit indices except at the residual invariance level. Proportion of invariance and ratio of sample size affected all fit indices at all levels of MI test. Also, the number of indicators affected RMSEA and Mc at all levels of tests. CFI and Gamma hat were affected by similar factors across all levels of tests and the percentages of variance explained by these factors were also similar for CFI and Gamma hat.

Based on the results obtained from the first part of the study and the significant factors and their interactions in the second part of the study, Chen (2007) recommended $\Delta$ CFI because of its wider use and high correlation with $\Delta$ Gamma hat. He also concludes that $\triangle$ RMSEA and 
$\Delta$ SRMR outperform $\Delta$ Mc. Under conditions that sample size is small (total $\mathrm{N}<300$ ), sample sizes are unequal, and the pattern of noninvariance is uniform, he suggested the following cut-off points: for testing factor loading invariance, a change of $\geq 0.005$ in CFI, supplemented by a change of $\geq .010$ in RMSEA or a change of $\geq .025$ in SRMR would indicate noninvariance; for testing intercept or residual invariance, a change of $\geq .005$ in CFI, supplemented by a change of $\geq .010$ in RMSEA or a change of $\geq .005$ in SRMR would indicate noninvariance. In other words, similar values are suggested for the CFI and the RMSEA across all three levels of invariance tests, but different values are proposed for SRMR, as SRMR is more sensitive to noninvariance in loadings than to noninvariance in intercepts or residual variances. When sample size is adequate (total $\mathrm{N}>300$ ) and sample sizes are equal across the groups, particularly when lack of invariance is mixed, more stringent criteria are suggested. For testing factor loading invariance, a change of $\geq .010$ in CFI, supplemented by a change of $\geq .015$ in RMSEA or a change of $\geq .030$ in SRMR would indicate noninvariance; for testing intercept or residual invariance, a change of $\geq$ .010 in CFI, supplemented by a change $\geq .015$ in RMSEA or a change $\geq .010$ in SRMR would indicate noninvariance. Among the three indexes, $\mathrm{CFI}$ was chosen as the main criterion because RMSEA and SRMR tend to overreject an invariant model when sample size is small, particularly when using SRMR for testing factor loading or residual variance invariance (Chen, 2007). In addition, changes in RMSEA are more likely to be affected by sample size and model complexity.

In a similar vein, Jin (2020) studied the appropriateness of the traditional cutoff values for CFI, TLI and RMSEA in a simulation study to evaluate measurement invariance in exploratory structural equation modeling (ESEM) with ordinal indicators. The motivation to verify these fit indices was that the previous cutoff values recommended by Cheung \& Rensvold 
(2002) were derived for confirmatory factor analysis models, not exploratory models. In other words, Jin (2020) attempted to evaluate the generality of those established cutoff values for a different setting, namely exploratory SEM and ordinal indicators. In her simulation study, Jin (2020) simulated different factor structures, with factor loadings in the range of 0.4 and 0.9 for all three factor structures, cross-loadings ranging from 0.2 to 0.3 (for smaller ones) and between 0.3 and 0.4 (for $30 \%$ of indicators). Indicator thresholds ranged from -2.0 to 2.0 (for both dichotomous and polytomous items). Factor scores were generated by $\mathrm{N}(0,1)$ and $\mathrm{N}(0,1)$ and $\mathrm{N}(-1,1)$ for the equal factor means MI level for both the focal and reference groups. Factor correlations ranged from 0.1 and 0.3 . The study conditions included sample size (500 and 1000), cross-loadings magnitude (small, medium large), factor means difference between groups (equal and nonequal), and type of indicators (dichotomous and polytomous). MI levels evaluated were metric and scalar invariance. The results of her study showed that the traditional cutoff values proposed by Cheung \& Rensvold (2002) were not sensitive to sample size, factor structure, and factor mean difference at both the metric and scalar noninvariance. For polytomous items, the cutoff values of the $\triangle \mathrm{CFI}=0.01$ and $\Delta \mathrm{TLI}=0.01$ showed some sensitivity at metric level to small and medium size cross-loadings in both equal and nonequal factor means condition, but not for cross-loadings which were large. The traditional $\Delta \mathrm{CFI}=0.01$ and $\Delta \mathrm{TLI}=0.01$ were not able to capture scalar invariance. In all conditions, RMSEA failed to capture scalar invariance, either. The author concludes that widely used change in fit indices $\Delta \mathrm{CFI}=0.01, \Delta \mathrm{TLI}=0.01$, and $\triangle \mathrm{RMSEA}=0.015$ were not able to detect scalar noninvariance in all study conditions, suggesting that different cutoff values of $\triangle \mathrm{CFI}, \triangle \mathrm{TLI}$, and $\triangle \mathrm{RMSEA}$ should be used for evaluating scalar invariance for dichotomous indicators. 
To supplement their study on the performance of different parameter estimators in the MG-CFA framework, Sass, Schmitt, and Marsh (2014), performed a simulation study on the appropriateness of the Cheung and Rensvold's (2002) cutoff criteria for establishing MI. They simulated a single-factor (mean zero) CFA model with 10 categorical indicators (with five categories) with factor loadings 0.60 (except on the noninvariant indicators), with a reliability of 0.83. For scaling purposes, indicator variance was constrained at one and the factor variance for both the focal and reference groups were set at one. Study factors included the shape of the distribution of the indicator thresholds (symmetric, asymmetric), three model estimators (MLE, MLR, WLSMV), sample sizes $(300,600,1000)$, and (only in the focal group) percentage of noninvariant items (10\% to $30 \%$ ). Configural, metric, and scalar noninvariance were simulated. The authors conclude that the proposed cutoff values (Chen, 2007; Meade et al., 2008) may not be appropriate for all conditions, especially when sample sizes are small and the magnitude of noninvariance (i.e. DIF) is also small.

In their study on the sensitivity of goodness-of-fit indices for detecting measurement invariance in a bifactor model, Khojasteh and Lo (2015) simulated a five-factor bifactor model with 20 normally distributed items. The simulation factors included sample size $(400,800$, 1200), magnitude of factor loading noninvariance (small and large), factors with noninvariant loadings, percentage of specific factors with noninvariant items $(50 \%, 100 \%)$, and percentage of noninvariant items $(20 \%, 40 \%)$. The results of the study showed that the cutoff values needed to determine lack of invariance not only differed across conditions, but also differed from the values proposed by Cheung and Rensvold (2002) and Chen (2007). 


\section{CHAPTER 3}

\section{METHOD}

Current research that has proposed cut-off values for change in fit indices for determining whether the lack of measurement invariance (MI) is non-negligible (e.g., $\Delta \mathrm{CFI}>$ 0.01) using MG-CFA have several limitations. First, the cut-off values have been recommended without considering the context and the purpose of the assessment. For example, previous research in this area have examined MI for indicators that are based on many categories where the items are treated as continuous. However, large-scale educational tests are often comprised of multiple-choice items that are dichotomously scored. The reason this is relevant is because the estimation method depends on the distribution and the type of indicators. Previous research has tested the indicators as continuous and, therefore, used maximum likelihood estimation (MLE) to estimate the parameters. For dichotomous indicators, however, we cannot use MLE, and instead, should use robust WLS. To address the lack of context and the purpose of the assessment in previous research, the current dissertation generated data using an IRT model (e.g., 3PL model), which is more realistic for educational data.

Second, when developing cut-off values, previous studies have ignored the consequences attached to the lack of invariance. In other words, if the LOI is present, would it lead to practically important consequences? Therefore, when determining if an LOI is non-negligible, it is important to consider the consequences due to the lack of MI. One way of defining the consequence is through the effect of MI on test scores. For example, if the MI resulted in examinees with the same proficiency but from different groups (e.g., based on testing mode) receiving a sufficiently different raw score, then the MI may be considered consequential. In the present study, non-negligible LOI was operationally defined as the difference in expected raw scores equal to 0.5 for examinees in different groups. We can determine the difference in 
expected raw scores by comparing test characteristic curves (TCC) across groups within an IRT framework. In other words, we are operationally defining non-negligible LOI if the difference in the TCCs between two groups is equal to 0.5 for any proficiency value. The rationale for the selection of a TCC difference of 0.5 is that such a difference would result in a one-point difference in raw score due to rounding error.

Third, although previous studies have examined several fit indices across multiple conditions, they have not addressed the effect of several important factors on the change in fit indices when determining an appropriate cut-off value. For example, is the change in fit indices (e.g., $\Delta$ CFI) influenced by item discrimination? Therefore, one of the purposes of the present study is to examine the impact of several important factors that may influence the distribution of the change in fit indices in assessing MI.

To address the three major limitations of the previous research, the present study employs a simulation to evaluate the appropriateness of the proposed cutoff value of $\Delta \mathrm{CFI}>$ 0.01 (Cheung \& Rensvold, 2002) in an educational measurement context and to examine the effects of several relevant factors on the change of fit indices for assessing MI using MG-CFA within an educational testing context. The details of the simulation follow.

\subsection{Design}

As stated above, one motivating rationale for conducting the present study was to investigate LOI and an effect size when the responses to the indicators are dichotomous. For this purpose, dichotomous item responses were generated for a 40-item test. A 40-item test was selected to represent a medium length test that is observed in many types of educational assessments. Responses to the 40-item test were generated under five crossed factors: generating model (two-parameter logistic (2PL) model and 3PL model), percentage of LOI items (10\% and 
$20 \%$ ), the $a$-parameter value for LOI items $(0.5,1.0$, and 1.5$)$, the $b$-parameter value for LOI items $(-1,0$, and 1$)$, and sample size per group $(500,1000$, and 2,000). These five factors yielded 108 study conditions. Because the present study used the multiple-group CFA approach, responses to the items were simulated for two groups, which are commonly known as the focal and the reference groups in differential item analysis literature.

To generate the item responses, two IRT models were used: the 3PL and the $2 \mathrm{PL}$ models. The 3PL model specifies the probability of a correct response given three item characteristics and a person parameter and is formulated as the following function:

$$
P\left(\theta_{j}\right)=c_{i}+\left(1-c_{i}\right) \frac{e^{1.7 a_{i}\left(\theta_{j}-b_{i}\right)}}{1+e^{1.7 a_{i}\left(\theta_{j}-b_{i}\right)}}
$$

where $b$ represents the item difficulty, $a$ represents the item discrimination, and $c$ represents the pseudo-guessing parameter. The 3PL model provides a realistic depiction of dichotomous item responses from a multiple-choice item. The 2PL model dispenses with the $c$ parameter but retains the $a$ and the $b$ parameters. The 2PL IRT model provides realistic item responses for short-answer items where guessing is minimal.

An advantage of using both the 3PL and 2PL models in this study is that I was able to examine the effect of model misspecification on the change in fit indices. In other words, MGCFA models the discrimination and difficulty of an item, but does not include a parameter to address answering the item correctly via guessing. Therefore, simulating the data using the 3PL 
model, although realistic, leads to model misspecification for the MG-CFA. It would be interesting to determine if the results differed between the 2PL and 3PL models.

Because the present study was based on simulating the data, item parameters calibrated using an IRT model were needed. In this study, parameters from a state-run proficiency test were used. In order to produce new parameters for the DIF items, parameters in the focal group were manipulated for each fixed parameter of the reference group.

The simulation steps for the present study proceeded as follows:

1. Item parameters estimated through an IRT model from a real test were obtained. In this study, item parameters were obtained from a large-scale assessment with 40 items and one construct dimension. The 3PL model was used to estimate the item parameters. The 40-item parameters were duplicated, one set for the reference group and the other set for the focal group. Therefore, there were two identical sets of item parameters with identical $a, b$, and $c$ parameters for 40 items.

2. The study condition was set according to the $a$ parameter value, the $b$ parameter value, the number of DIF items, the IRT model, and the sample size. Study conditions related to the item were only applied to the DIF item(s), leaving all other item parameters intact. For example, the first condition could be:

- number of DIF items: four

- IRT model: 3PL (hence all three item parameters values are used)

- $a=0.5$ (both in the reference and the focal group)

- $\quad b=-1$ (only in the reference group)

- $c=.20$ (both in the reference and the focal group)

- $\quad$ sample size $=500$ (both in the reference and the focal group) 
3. Once the parameters for a study condition were set, the value of the $b$ parameter of the DIF item was manipulated only in the focal group to produce an amount of DIF due to which the difference between the test characteristic curves (TCC) in the reference and the focal groups stays at 0.5 . A program was run to calculate the TCC values in both groups and their possible difference after the introduction of the DIF item in the focal group. For instance, for the condition parameters set in Step 2 above, a $b$ value of -2.263 is needed such that the TCC values in the reference group and the focal group differ by .5 .

4. Once the value of the $b$ parameter of the target DIF item in the focal group was achieved such that the TCC difference between the two groups is maximum of .5, a program was used to generate item responses based on the changed parameter values in the original 40 -item sets.

5. After item responses for all 108 study conditions were simulated and generated, the obtained item responses were used in the Mplus software for multiple-group CFA study in order to investigate the effect of different conditions on the obtained effect sizes (CFI, TLI, and RMSEA).

Table 4.2 shows the different $b$ values in the focal group used to simulate DIF items for different conditions, including different values of the $a$ parameter, different values of the $b$ parameters in the reference group, the proportion of DIF items, and model complexity. 
Table 2. The $b$ parameter values used to obtain LOI items in the focal group.

\begin{tabular}{|c|c|c|c|c|c|}
\hline & & \multicolumn{2}{|c|}{ 2PL Model } & \multicolumn{2}{|c|}{ 3PL Model } \\
\hline & & $10 \%$ LOI & $20 \%$ LOI & $10 \%$ LOI & $20 \%$ LOI \\
\hline \multirow[t]{2}{*}{$a_{\text {Reference }}=a_{\text {Focal }}$} & $b_{\text {Reference }}$ & $b_{\text {Focal }}$ & $b_{\text {Focal }}$ & $b_{\text {Focal }}$ & $b_{\text {Focal }}$ \\
\hline & -1.00 & -2.000 & -1.501 & -2.263 & -1.630 \\
\hline \multirow[t]{3}{*}{0.5} & 0.00 & 1.006 & 0.501 & 1.260 & 0.630 \\
\hline & 1.00 & -0.006 & 1.501 & 2.263 & 1.630 \\
\hline & -1.00 & -1.502 & -0.750 & -1.629 & -0.687 \\
\hline \multirow[t]{3}{*}{1.0} & 0.00 & 0.503 & 0.250 & 0.631 & 0.313 \\
\hline & 1.00 & 1.502 & 1.250 & 1.629 & 1.313 \\
\hline & -1.00 & -0.664 & -1.167 & -0.579 & -1.209 \\
\hline \multirow[t]{2}{*}{1.5} & 0.00 & 0.335 & 0.167 & 0.420 & 0.209 \\
\hline & 1.00 & 0.664 & 1.167 & 0.579 & 1.209 \\
\hline
\end{tabular}

The $a$ - and $b$-parameter values for the items for the reference group selected to exhibit an LOI were manipulated to examine the effect of item discrimination and difficulty on the change in fit indices. Three values of the $a$-parameter $(0.5,1.0$, and 1.5$)$ were used for the items that exhibited an LOI and were crossed with three values of the $b$-parameter value $(-1,0$, and 1$)$. Manipulating the $a$ - and the $b$-parameters in this manner allowed me to determine if the distribution of the change in fit indices was influenced by the item characteristics.

In addition, data were simulated for three different sample sizes per group conditions: 500,1000 , and 2,000. The purpose of manipulating the sample sizes per group was to examine 
the effect on the distribution of the change in fit indices as a function of sample size. Overall, there were 108 conditions and 1,000 replications per condition.

\subsection{Parameter Estimation}

A multi-group confirmatory factor analysis (MG-CFA) was conducted on the generated data. Sequential equality constraints were placed on factor structure, factor loadings, and indicator thresholds for the purpose of testing measurement invariance at factor, metric and scalar levels, respectively. At each level, the CFI, TLI, and the RMSEA fit indices were estimated and compared to the higher-order nested model to see the amount of deterioration in fit due to equality constraints.

To estimate the model parameters, the simulated data generated for the reference group and the focal group were combined but aggregated by a grouping variable and input into the Mplus v.5.0 software. Because the data were dichotomous, robust diagonally weighted least squares (WLSMV; Muthen et al., 1997) was used to estimate the model parameters. One nonDIF item was selected as the referent indicator for scaling the latent variable in both groups.

To test the configural invariance, the factor structure was the same (i.e., one-factor) in both groups, but the factor loadings, thresholds, and residual variances were unconstrained. To test metric invariance, the factor loadings were constrained to be equal between the two groups. And finally, to test scalar invariance, the factor loadings and the thresholds were constrained between the two groups. Therefore, in each condition, three models with three equality constraints were run and the values of the CFI, TLI, and the RMSEA fit indices recorded and subtracted from the higher order model to see the amount of change in fit. To facilitate model running, the $R$ package Mplus Automation (Hallquist, 2017) was used to batch-process the sequential models. 
Because I was only introducing an LOI using the $b$-parameter values, the fit of the model should only deteriorate when placing constraints on the thresholds.

\subsection{Data Analysis}

The purpose of the present study was to compare the distributions of the three fit indices ( $\triangle \mathrm{CFI}, \triangle \mathrm{TLI}$, and $\triangle \mathrm{RMSEA}$ ) that are often used to assess non-negligible measurement invariance under various conditions within an educational context. In particular, I intended to examine the mean, standard deviation, skewness, and kurtosis for each fit index. Lastly, since the mean of the fit indices would provide an estimate of the cut-off value that could be used to determine non-negligible LOI, a five-way ANOVA was run to determine the important factors that may influence the mean of the distributions. An effect size based on partial eta-squared was used to identify effects that are practically meaningful. Because the conditions are the same with respect to the consequences of including LOI in the assessment, for the fit indices to be useful their expected value should remain the same across the conditions. 


\section{CHAPTER 4}

\section{RESULTS}

\subsection{Introduction}

The simulation results for the current study are presented in this chapter. In this study, the CFI, TLI, and the RMSEA model fit indices were examined in the multiple-group confirmatory factor analysis (MG-CFA) framework to investigate their behavior in the presence of non-negligible measurement invariance induced by 0.5 difference in test characteristic curve (TCC) between the focal and the reference groups on a one-dimensional IRT-calibrated test with 40 dichotomous items. The purpose of the study was to evaluate the appropriateness of the effect size criterion set in the current practice for the CFI, TLI, and the RMSEA fit indices in capturing scalar measurement invariance when the items are dichotomous and parameters are estimated using robust weighted least squares. In addition, the simulation study investigated the effect of DIF percentage, IRT models, the $a$ and $b$ parameter values, and the sample size on the change in the CFI, TLI, and the RMSEA model fit indices. Overall 108 conditions were simulated and investigated.

\subsection{Validating Simulation Data}

Before proceeding to examine the distributions for each of the changes in goodness-offit indices, I used the Mantel-Haenszel (MH) DIF procedure to flag items for a few select conditions to ensure the data were generated appropriately. If the simulation was correct, I expected the "DIF" items to be flagged at a much higher rate than the non-DIF items and the average effect size to be greater for the DIF items compared to the non-DIF items. Also, I expected the non-DIF items not to be flagged much beyond a nominal alpha level of .05. The MH DIF procedure was applied to three conditions where the $a$-parameter varied (i.e., $a=0.5, a$ 
$=1.0$, and $a=1.5$ ) when generating data using the 2PLM and a sample size of 1000. Table 4.1 shows the proportion of replications that each simulated DIF item was flagged as DIF and the mean effect size, $\triangle \mathrm{MH}$ for the selected conditions. Because the MH DIF method was able to detect the DIF items with reasonable power, it seems that the items were generated appropriately. Furthermore, the average $\Delta \mathrm{MH}$ across the items was reasonably large for all of the DIF items.

Table 4.1

Proportions of Simulated DIF Items Detected by Mantel-Haenszel Test when IRT model = 2PL, DIF percentage $=20 \%$

\begin{tabular}{|c|c|c|c|c|c|c|}
\hline \multirow[b]{2}{*}{ Item } & \multicolumn{2}{|c|}{$a=0.5, b=0$} & \multicolumn{2}{|c|}{$a=1.0, b=0$} & \multicolumn{2}{|c|}{$a=1.5, b=0$} \\
\hline & $\begin{array}{c}\text { Proportion } \\
\text { Flagged }\end{array}$ & $\begin{array}{c}\text { Mean Delta } \\
\text { MH }\end{array}$ & $\begin{array}{c}\text { Proportion } \\
\text { Flagged }\end{array}$ & $\begin{array}{c}\text { Mean Delta } \\
\text { MH }\end{array}$ & $\begin{array}{c}\text { Proportion } \\
\text { Flagged }\end{array}$ & $\begin{array}{c}\text { Mean Delta } \\
M H\end{array}$ \\
\hline 1 & 0.74 & 0.52 & 0.49 & 0.47 & 0.35 & 0.45 \\
\hline 2 & 0.72 & 0.51 & 0.49 & 0.47 & 0.33 & 0.44 \\
\hline 3 & 0.72 & 0.51 & 0.50 & 0.48 & 0.36 & 0.45 \\
\hline 4 & 0.77 & 0.52 & 0.50 & 0.47 & 0.31 & 0.43 \\
\hline 5 & 0.75 & 0.52 & 0.49 & 0.48 & 0.35 & 0.45 \\
\hline 6 & 0.75 & 0.52 & 0.51 & 0.48 & 0.36 & 0.46 \\
\hline 7 & 0.74 & 0.52 & 0.50 & 0.48 & 0.36 & 0.45 \\
\hline 8 & 0.74 & 0.52 & 0.50 & 0.47 & 0.35 & 0.44 \\
\hline $\begin{array}{l}9-40 \\
\text { (Non- } \\
\text { DIF) }\end{array}$ & 0.02 & -0.17 & 0.01 & -0.13 & 0.01 & -0.11 \\
\hline
\end{tabular}




\subsection{ANOVA Results}

A five-way ANOVA was performed to investigate if there were any differences

between the factors in terms of their effect on the amount of change in each fit index. The

following ANOVA tables (changes in CFI, the TLI, and the RMSEA) show that the differences

were statistically significant, though it is more appropriate to refer to the partial eta squared

values to evaluate the effect of the factors and their interactions.

Table 4.2

Five-way ANOVA on CFI Change across the Five Factors

\begin{tabular}{|c|c|c|c|c|c|c|}
\hline Source of Variation & Sum of Squares & df & $\begin{array}{l}\text { Mean } \\
\text { Square }\end{array}$ & $\mathrm{F}$ & Sig & Partial Eta Squared \\
\hline Sample Size & 0.007 & 2 & 0.003 & 3145.0 & $<.001$ & 0.06 \\
\hline IRT Model & 0.002 & 1 & 0.002 & 2298.0 & $<.001$ & 0.02 \\
\hline Percent DIF & 0.003 & 1 & 0.003 & 3113.7 & $<.001$ & 0.03 \\
\hline Parameter "a" & 0.004 & 2 & 0.002 & 1608.5 & $<.001$ & 0.03 \\
\hline Parameter "b" & 0.003 & 2 & 0.001 & 1226.5 & $<.001$ & 0.02 \\
\hline Sample Size X IRT Model & 0.002 & 2 & 0.001 & 1020.6 & $<.001$ & 0.02 \\
\hline Sample Size X Percent DIF & 0.003 & 2 & 0.001 & 1287.6 & $<.001$ & 0.02 \\
\hline IRT Model X Percent DIF & 0.009 & 1 & 0.009 & 8262.1 & $<.001$ & 0.07 \\
\hline Sample Size X Parameter "a" & 0.004 & 4 & 0.001 & 927.4 & $<.001$ & 0.03 \\
\hline IRT Model X Parameter “a” & 0.009 & 2 & 0.004 & 4153.3 & $<.001$ & 0.07 \\
\hline Percent DIF X Parameter “a” & 0.007 & 2 & 0.003 & 3207.9 & $<.001$ & 0.06 \\
\hline Sample Size X Parameter "b" & 0.005 & 4 & 0.001 & 1133.9 & $<.001$ & 0.04 \\
\hline IRT Model X Parameter “b” & 0.002 & 2 & 0.001 & 1089.5 & $<.001$ & 0.02 \\
\hline Percent DIF X Parameter "b" & 0.002 & 2 & 0.001 & 825.1 & $<.001$ & 0.02 \\
\hline Parameter "a" X Parameter "b" & 0.004 & 4 & 0.001 & 1040.4 & $<.001$ & 0.02 \\
\hline $\begin{array}{l}\text { Sample Size X IRT Model X Percent } \\
\text { DIF }\end{array}$ & 0.002 & 2 & 0.001 & 972.4 & $<.001$ & 0.02 \\
\hline $\begin{array}{l}\text { Sample Size X IRT Model X } \\
\text { Parameter "a" }\end{array}$ & 0.006 & 4 & 0.001 & 1381.6 & $<.001$ & 0.05 \\
\hline $\begin{array}{l}\text { Sample Size X Percent DIF X } \\
\text { Parameter "a" }\end{array}$ & 0.005 & 4 & 0.001 & 1130.1 & $<.001$ & 0.04 \\
\hline $\begin{array}{l}\text { IRT Model X Percent DIF X } \\
\text { Parameter "a" }\end{array}$ & 0.003 & 2 & 0.001 & 1308.6 & $<.001$ & 0.02 \\
\hline $\begin{array}{l}\text { Sample Size X IRT Model X } \\
\text { Parameter "b" }\end{array}$ & 0.004 & 4 & 0.001 & 968.9 & $<.001$ & 0.03 \\
\hline $\begin{array}{l}\text { Sample Size X Percent DIF X } \\
\text { Parameter "b" }\end{array}$ & 0.005 & 4 & 0.001 & 1086.5 & $<.001$ & 0.04 \\
\hline $\begin{array}{l}\text { IRT Model X Percent DIF X } \\
\text { Parameter "b" }\end{array}$ & 0.002 & 2 & 0.001 & 1084.6 & $<.001$ & 0.02 \\
\hline $\begin{array}{l}\text { Sample Size X Parameter "a" X } \\
\text { Parameter "b" }\end{array}$ & 0.009 & 8 & 0.001 & 1011.4 & $<.001$ & 0.07 \\
\hline $\begin{array}{l}\text { IRT Model X Parameter "a" X } \\
\text { Parameter "b" }\end{array}$ & 0.005 & 4 & 0.001 & 1066.6 & $<.001$ & 0.04 \\
\hline $\begin{array}{l}\text { Percent DIF X Parameter "a" X } \\
\text { Parameter "b" }\end{array}$ & 0.005 & 4 & 0.001 & 1165.6 & $<.001$ & 0.04 \\
\hline $\begin{array}{l}\text { Sample Size X IRT Model X Percent } \\
\text { DIF X Parameter "a" }\end{array}$ & 0.008 & 4 & 0.002 & 1799.4 & $<.001$ & 0.07 \\
\hline $\begin{array}{l}\text { Sample Size X IRT Model X Percent } \\
\text { DIF X Parameter "b" }\end{array}$ & 0.005 & 4 & 0.001 & 1102.6 & $<.001$ & 0.04 \\
\hline $\begin{array}{l}\text { Sample Size X IRT Model X } \\
\text { Parameter "a" X Parameter "b" }\end{array}$ & 0.009 & 8 & 0.001 & 1105.4 & $<.001$ & 0.08 \\
\hline
\end{tabular}




\begin{tabular}{|c|c|c|c|c|c|c|}
\hline $\begin{array}{l}\text { Sample Size X Percent DIF X } \\
\text { Parameter "a" X Parameter "b" }\end{array}$ & 0.010 & 8 & 0.001 & 1107.0 & $<.001$ & 0.08 \\
\hline $\begin{array}{l}\text { IRT Model X Percent DIF X } \\
\text { Parameter “a" X Parameter “b” }\end{array}$ & 0.005 & 4 & 0.001 & 1114.7 & $<.001$ & 0.04 \\
\hline $\begin{array}{l}\text { Sample Size X IRT Model X Percent } \\
\text { DIF X Parameter "a" X Parameter } \\
\text { "b" }\end{array}$ & 0.009 & 8 & 0.001 & 1017.2 & $<.001$ & 0.07 \\
\hline Error & 0.116 & $\begin{array}{l}107892 \\
107999\end{array}$ & $<.001$ & & & \\
\hline
\end{tabular}

\section{Table 4.3}

Five-way ANOVA on TLI Change across the Five Factors

\begin{tabular}{|c|c|c|c|c|c|c|}
\hline Source of Variation & Sum of Squares & $\mathrm{df}$ & $\begin{array}{l}\text { Mean } \\
\text { Square }\end{array}$ & $\mathrm{F}$ & Sig & Partial Eta Squared \\
\hline Sample Size & 0.041 & 2 & 0.021 & 6082.8 & $<.001$ & 0.10 \\
\hline IRT Model & 0.002 & 1 & 0.002 & 533.1 & $<.001$ & 0.00 \\
\hline Percent DIF & 0.020 & 1 & 0.020 & 6026.1 & $<.001$ & 0.05 \\
\hline Parameter "a" & 0.029 & 2 & 0.015 & 4332.5 & $<.001$ & 0.07 \\
\hline Parameter "b" & 0.007 & 2 & 0.004 & 1104.7 & $<.001$ & 0.02 \\
\hline Sample Size X IRT Model & 0.009 & 2 & 0.004 & 1329.9 & $<.001$ & 0.02 \\
\hline Sample Size X Percent DIF & 0.001 & 2 & 0.001 & 217.5 & $<.001$ & 0.00 \\
\hline IRT Model X Percent DIF & $<.001$ & 1 & $<.001$ & 15.7 & $<.001$ & 0.00 \\
\hline Sample Size X Parameter "a" & 0.005 & 4 & 0.001 & 344.5 & $<.001$ & 0.01 \\
\hline IRT Model X Parameter "a" & 0.000 & 2 & $<.001$ & 25.5 & $<.001$ & 0.00 \\
\hline Percent DIF X Parameter "a" & 0.004 & 2 & 0.002 & 534.5 & $<.001$ & 0.01 \\
\hline Sample Size X Parameter "b" & 0.008 & 4 & 0.002 & 600.5 & $<.001$ & 0.02 \\
\hline IRT Model X Parameter "b" & 0.004 & 2 & 0.002 & 654.2 & $<.001$ & 0.01 \\
\hline Percent DIF X Parameter "b" & 0.001 & 2 & 0.001 & 183.8 & $<.001$ & 0.00 \\
\hline Parameter "a" X Parameter "b" & 0.006 & 4 & 0.002 & 474.4 & $<.001$ & 0.02 \\
\hline $\begin{array}{l}\text { Sample Size X IRT Model X Percent } \\
\text { DIF }\end{array}$ & 0.001 & 2 & 0.001 & 203.5 & $<.001$ & 0.00 \\
\hline $\begin{array}{l}\text { Sample Size X IRT Model X } \\
\text { Parameter "a" }\end{array}$ & 0.015 & 4 & 0.004 & 1080.1 & $<.001$ & 0.04 \\
\hline $\begin{array}{l}\text { Sample Size X Percent DIF X } \\
\text { Parameter "a" }\end{array}$ & 0.021 & 4 & 0.005 & 1565.0 & $<.001$ & 0.06 \\
\hline $\begin{array}{l}\text { IRT Model X Percent DIF X } \\
\text { Parameter "a" }\end{array}$ & 0.001 & 2 & 0.001 & 170.5 & $<.001$ & 0.00 \\
\hline $\begin{array}{l}\text { Sample Size X IRT Model X } \\
\text { Parameter "b" }\end{array}$ & 0.009 & 4 & 0.002 & 653.2 & $<.001$ & 0.02 \\
\hline $\begin{array}{l}\text { Sample Size X Percent DIF X } \\
\text { Parameter "b" }\end{array}$ & 0.012 & 4 & 0.003 & 880.9 & $<.001$ & 0.03 \\
\hline $\begin{array}{l}\text { IRT Model X Percent DIF X } \\
\text { Parameter "b" }\end{array}$ & 0.004 & 2 & 0.002 & 621.7 & $<.001$ & 0.01 \\
\hline $\begin{array}{l}\text { Sample Size X Parameter "a" X } \\
\text { Parameter "b" }\end{array}$ & 0.012 & 8 & 0.001 & 440.5 & $<.001$ & 0.03 \\
\hline $\begin{array}{l}\text { IRT Model X Parameter "a" X } \\
\text { Parameter "b" }\end{array}$ & 0.008 & 4 & 0.002 & 562.4 & $<.001$ & 0.02 \\
\hline $\begin{array}{l}\text { Percent DIF X Parameter "a" X } \\
\text { Parameter "b" }\end{array}$ & 0.008 & 4 & 0.002 & 621.9 & $<.001$ & 0.02 \\
\hline $\begin{array}{l}\text { Sample Size X IRT Model X Percent } \\
\text { DIF X Parameter "a" }\end{array}$ & 0.022 & 4 & 0.006 & 1663.8 & $<.001$ & 0.06 \\
\hline $\begin{array}{l}\text { Sample Size X IRT Model X Percent } \\
\text { DIF X Parameter "b" }\end{array}$ & 0.006 & 4 & 0.002 & 481.2 & $<.001$ & 0.02 \\
\hline $\begin{array}{l}\text { Sample Size X IRT Model X } \\
\text { Parameter "a" X Parameter "b" }\end{array}$ & 0.010 & 8 & 0.001 & 359.4 & $<.001$ & 0.03 \\
\hline $\begin{array}{l}\text { Sample Size X Percent DIF X } \\
\text { Parameter "a" X Parameter "b" }\end{array}$ & 0.011 & 8 & 0.001 & 393.7 & $<.001$ & 0.03 \\
\hline $\begin{array}{l}\text { IRT Model X Percent DIF X } \\
\text { Parameter "a" X Parameter “b” }\end{array}$ & 0.011 & 4 & 0.003 & 847.1 & $<.001$ & 0.03 \\
\hline $\begin{array}{l}\text { Sample Size X IRT Model X Percent } \\
\text { DIF X Parameter "a" X Parameter } \\
\text { "b" }\end{array}$ & 0.013 & 8 & 0.002 & 493.7 & $<.001$ & 0.04 \\
\hline Error & 0.364 & 107892 & $<.001$ & & & \\
\hline Total & 0.668 & 107999 & & & & \\
\hline
\end{tabular}


Table 4.4

Five-way ANOVA on RMSEA Change across the Five Factors

\begin{tabular}{|c|c|c|c|c|c|c|}
\hline Source of Variation & Sum of Squares & df & $\begin{array}{l}\text { Mean } \\
\text { Square }\end{array}$ & $\mathrm{F}$ & Sig & Partial Eta Squared \\
\hline Sample Size & 0.029 & 2 & 0.015 & 4124.2 & $<.001$ & 0.07 \\
\hline IRT Model & 0.031 & 1 & 0.031 & 8822.5 & $<.001$ & 0.08 \\
\hline Percent DIF & 0.026 & 1 & 0.026 & 7308.1 & $<.001$ & 0.06 \\
\hline Parameter "a" & 0.012 & 2 & 0.006 & 1766.1 & $<.001$ & 0.03 \\
\hline Parameter "b" & 0.003 & 2 & 0.001 & 373.8 & $<.001$ & 0.01 \\
\hline Sample Size X IRT Model & 0.006 & 2 & 0.003 & 860.6 & $<.001$ & 0.02 \\
\hline Sample Size X Percent DIF & 0.006 & 2 & 0.003 & 808.7 & $<.001$ & 0.02 \\
\hline IRT Model X Percent DIF & 0.027 & 1 & 0.027 & 7528.1 & $<.001$ & 0.07 \\
\hline Sample Size X Parameter "a" & 0.002 & 4 & 0.001 & 165.7 & $<.001$ & 0.01 \\
\hline IRT Model X Parameter "a" & 0.019 & 2 & 0.009 & 2636.5 & $<.001$ & 0.05 \\
\hline Percent DIF X Parameter "a" & 0.010 & 2 & 0.005 & 1438.5 & $<.001$ & 0.03 \\
\hline Sample Size X Parameter "b" & 0.005 & 4 & 0.001 & 380.5 & $<.001$ & 0.01 \\
\hline IRT Model X Parameter "b" & 0.002 & 2 & 0.001 & 335.0 & $<.001$ & 0.01 \\
\hline Percent DIF X Parameter "b" & 0.002 & 2 & 0.001 & 222.1 & $<.001$ & 0.00 \\
\hline Parameter "a" X Parameter "b" & 0.005 & 4 & 0.001 & 356.5 & $<.001$ & 0.01 \\
\hline $\begin{array}{l}\text { Sample Size X IRT Model X Percent } \\
\text { DIF }\end{array}$ & 0.003 & 2 & 0.001 & 414.2 & $<.001$ & 0.01 \\
\hline $\begin{array}{l}\text { Sample Size X IRT Model X } \\
\text { Parameter "a" }\end{array}$ & 0.010 & 4 & 0.003 & 735.8 & $<.001$ & 0.03 \\
\hline $\begin{array}{l}\text { Sample Size X Percent DIF X } \\
\text { Parameter "a" }\end{array}$ & 0.006 & 4 & 0.002 & 446.7 & $<.001$ & 0.02 \\
\hline $\begin{array}{l}\text { IRT Model X Percent DIF X } \\
\text { Parameter "a" }\end{array}$ & 0.008 & 2 & 0.004 & 1082.7 & $<.001$ & 0.02 \\
\hline $\begin{array}{l}\text { Sample Size X IRT Model X } \\
\text { Parameter "b" }\end{array}$ & 0.003 & 4 & 0.001 & 201.4 & $<.001$ & 0.08 \\
\hline $\begin{array}{l}\text { Sample Size X Percent DIF X } \\
\text { Parameter "b" }\end{array}$ & 0.006 & 4 & 0.001 & 414.9 & $<.001$ & 0.02 \\
\hline $\begin{array}{l}\text { IRT Model X Percent DIF X } \\
\text { Parameter "b" }\end{array}$ & 0.002 & 2 & 0.001 & 270.4 & $<.001$ & 0.00 \\
\hline $\begin{array}{l}\text { Sample Size X Parameter "a" X } \\
\text { Parameter "b" }\end{array}$ & 0.010 & 8 & 0.001 & 337.6 & $<.001$ & 0.02 \\
\hline $\begin{array}{l}\text { IRT Model X Parameter "a" X } \\
\text { Parameter "b" }\end{array}$ & 0.004 & 4 & 0.001 & 304.5 & $<.001$ & 0.01 \\
\hline $\begin{array}{l}\text { Percent DIF X Parameter "a" X } \\
\text { Parameter "b" }\end{array}$ & 0.005 & 4 & 0.001 & 328.4 & $<.001$ & 0.01 \\
\hline $\begin{array}{l}\text { Sample Size X IRT Model X Percent } \\
\text { DIF X Parameter "a" }\end{array}$ & 0.014 & 4 & 0.004 & 1009.2 & $<.001$ & 0.04 \\
\hline $\begin{array}{l}\text { Sample Size X IRT Model X Percent } \\
\text { DIF X Parameter "b" }\end{array}$ & 0.006 & 4 & 0.001 & 369.0 & $<.001$ & 0.01 \\
\hline $\begin{array}{l}\text { Sample Size X IRT Model X } \\
\text { Parameter "a" X Parameter "b" }\end{array}$ & 0.012 & 8 & 0.001 & 419.7 & $<.001$ & 0.03 \\
\hline $\begin{array}{l}\text { Sample Size X Percent DIF X } \\
\text { Parameter "a" X Parameter "b" }\end{array}$ & 0.010 & 8 & 0.001 & 343.5 & $<.001$ & 0.02 \\
\hline $\begin{array}{l}\text { IRT Model X Percent DIF X } \\
\text { Parameter "a" X Parameter "b" }\end{array}$ & 0.006 & 4 & 0.001 & 399.5 & $<.001$ & 0.01 \\
\hline $\begin{array}{l}\text { Sample Size X IRT Model X Percent } \\
\text { DIF X Parameter "a" X Parameter } \\
\text { "b" }\end{array}$ & 0.009 & 8 & 0.001 & 315.7 & $<.001$ & 0.02 \\
\hline Error & 0.380 & 107892 & $<.001$ & & & \\
\hline Total & 0.782 & 107999 & & & & \\
\hline
\end{tabular}




\subsection{Descriptive Results}

The results of simulation with 1000 replications are presented in the following tables. The tables represent different study conditions. The conditions include the percentage of DIF items in the 40 -item test (10\% and $20 \%$ ), the IRT model (the 2PL and the 3PL models), the $b$ parameter $(-1,0,1)$, the $a$ parameter $(0.5,1.0,1.5)$, and the sample size $(500,1000,2000)$. Tables 4.5 to 4.10 show the change in the model fit indices for the equal factor loadings and equal threshold constrained CFI model (the scalar invariance), which is the main interest of the present study.

Table 4.5 and Figure 4.1 show the GFI changes when the sample size was 500, and the IRT model is the 3PL. Although the MH test procedure showed the presence of DIF items in the simulated test scores, the CFI, TLI, and the RMSEA seem to fail in capturing model fit deterioration due to the existence of measurement noninvariance at the scalar level. As the different GFI change values show, they are much smaller than the recommended 0.01 change in CFI and TLI suggested by Cheng and Rensvold (2002). At a given value of $b$, we can see that as the $a$ parameter increased, the changes in both the CFI and the TLI became smaller, indicating the offsetting contribution of the $a$ parameter in the presence of DIF. A similar change can also be observed for the RMSEA. For instance, at $b=0$, the RMSEA change decreased from 0.005 to 0.003 and to 0.001 for $a$ parameter values of $0.5,1.0$, and 1.5 , respectively. However, the change is positive, which is unexpected. The changes in GFI when the DFI percentage is $20 \%$ are more stable. We do not see any noticeable change from one condition to another. However, the change in GFI is largest at the lowest level of the discrimination parameter. This once again shows the offsetting role of the $a$ parameter in the presence of DIF. 
Table 4.5

Change in Equal Loadings Equal Threshold (Scalar Invariance) GFI across conditions for $N=500, I R T=3 P L$

\begin{tabular}{|c|c|c|c|c|c|c|c|c|c|c|}
\hline \multicolumn{2}{|c|}{ 3PL IRT } & \multicolumn{3}{|c|}{$b=-1$} & \multicolumn{3}{|c|}{$\mathbf{b}=\mathbf{0}$} & \multicolumn{3}{|c|}{$b=1$} \\
\hline & & $a=0.5$ & $a=1.0$ & $a=1.5$ & $a=0.5$ & $a=1.0$ & $a=1.5$ & $a=0.5$ & $a=1.0$ & $a=1.5$ \\
\hline \multirow[t]{3}{*}{$10 \%$} & CFI & -0.002 & -0.001 & -0.000 & -0.003 & -0.001 & -0.000 & -0.002 & -0.001 & -0.000 \\
\hline & TLI & -0.003 & -0.001 & -0.000 & -0.003 & -0.002 & -0.000 & -0.003 & -0.001 & -0.000 \\
\hline & RMSEA & 0.007 & -0.001 & 0.001 & 0.005 & 0.003 & 0.001 & 0.004 & 0.002 & 0.001 \\
\hline \multirow[t]{3}{*}{$20 \%$} & CFI & -0.001 & -0.000 & -0.000 & -0.001 & -0.000 & -0.000 & -0.001 & -0.000 & -0.000 \\
\hline & TLI & -0.001 & -0.000 & -0.000 & -0.001 & -0.000 & -0.000 & -0.001 & -0.000 & -0.000 \\
\hline & RMSEA & 0.001 & 0.000 & 0.000 & 0.001 & 0.000 & 0.000 & 0.001 & 0.000 & 0.000 \\
\hline
\end{tabular}

CFI change, $\mathrm{N}=500$, DIF=10\%, IRT=3PL

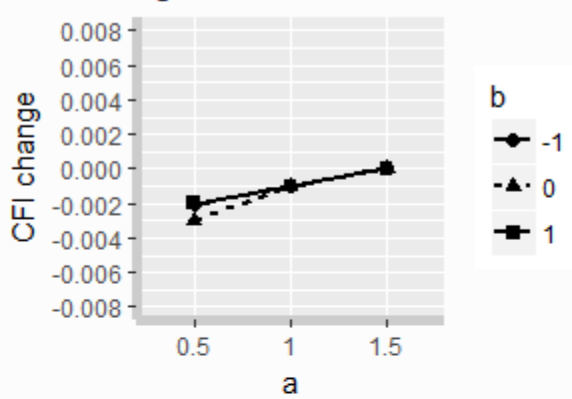

CFI change, $\mathrm{N}=500$, DIF $=20 \%, \mathrm{IR} T=3 \mathrm{PL}$

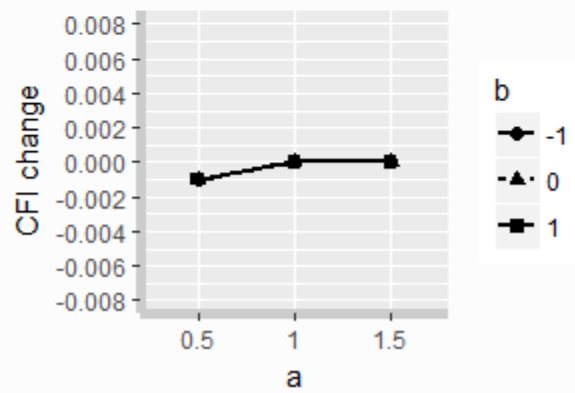

TLI change, $N=500$, DIF=10\%, IRT=3PL

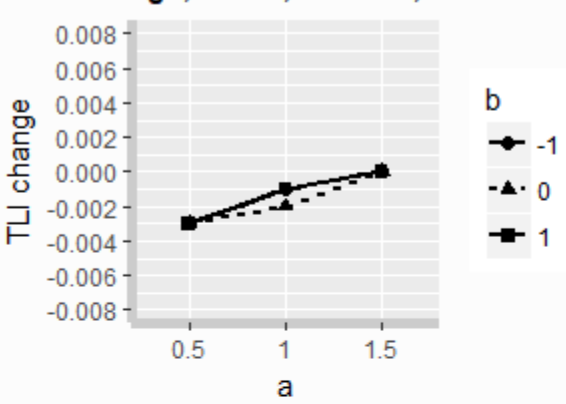

TLI change, $N=500$, DIF $=20 \%$, IRT $=3 P L$

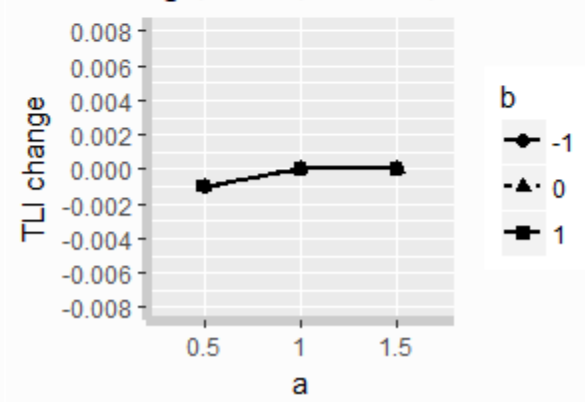

RMSEA change, N=500, DIF=10\%, IRT=3PL

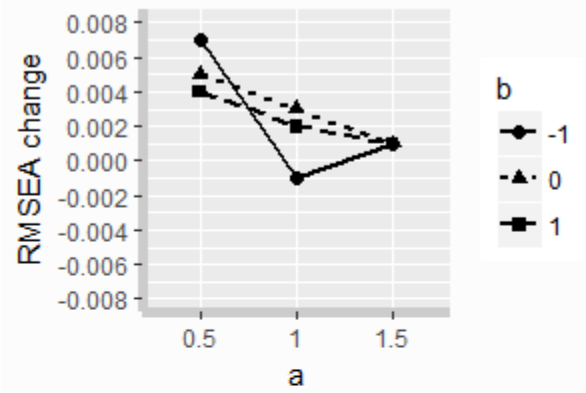

RMSEA change, $N=500$, DIF $=20 \%$, IRT=3PL

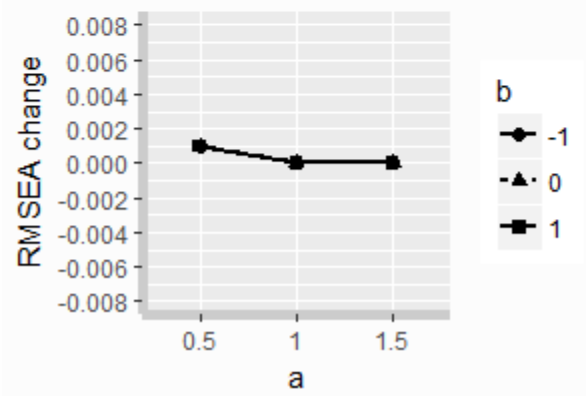

Figure 4.1. Change in equal loadings, equal threshold (scalar invariance) GFI's across conditions for $\mathrm{N}=500$, IRT=3PL. 
Table 4.6 and Figure 4.2 show the results of the simulation for the sample size of 500 but the 2PL IRT model. Similar to the 3PL model, we can see that the CFI, TLI, and the RMSEA all fail to capture the simulated scalar noninvariance. Once again the effect of increased $a$ parameter is observed across the $b$ values but only in the TLI changes. However, the effect in this case is the reverse because as the $a$ parameter increases, the change in TLI also increases. When the percentage of DIF is $20 \%$, however, the TLI seems to remain constant across most conditions.

Table 4.6

Change in Equal Loadings Equal Threshold (Scalar Invariance) GFI across conditions for $N=500, I R T=2 P L$

\begin{tabular}{|c|c|c|c|c|c|c|c|c|c|c|}
\hline \multicolumn{2}{|c|}{ 2PL IRT } & \multicolumn{3}{|c|}{$b=-1$} & \multicolumn{3}{|c|}{$b=0$} & \multicolumn{3}{|c|}{$b=1$} \\
\hline & & $\mathrm{a}=0.5$ & $a=1.0$ & $\mathrm{a}=1.5$ & $a=0.5$ & $\mathrm{a}=1.0$ & $a=1.5$ & $a=0.5$ & $a=1.0$ & $a=1.5$ \\
\hline \multirow[t]{3}{*}{$10 \%$} & CFI & -0.000 & 0.000 & -0.000 & -0.000 & -0.000 & -0.000 & -0.000 & 0.000 & -0.000 \\
\hline & TLI & -0.000 & 0.000 & 0.001 & -0.000 & 0.000 & 0.001 & -0.000 & 0.001 & 0.002 \\
\hline & RMSEA & -0.000 & -0.000 & -0.000 & -0.000 & -0.000 & -0.000 & -0.000 & -0.000 & -0.000 \\
\hline \multirow[t]{3}{*}{$20 \%$} & CFI & -0.000 & -0.000 & -0.000 & -0.000 & -0.000 & -0.000 & -0.000 & -0.000 & -0.000 \\
\hline & TLI & 0.002 & 0.000 & 0.002 & 0.002 & 0.002 & 0.002 & 0.002 & 0.003 & 0.003 \\
\hline & RMSEA & -0.000 & -0.000 & -0.000 & -0.000 & -0.000 & -0.000 & -0.000 & -0.000 & -0.000 \\
\hline
\end{tabular}



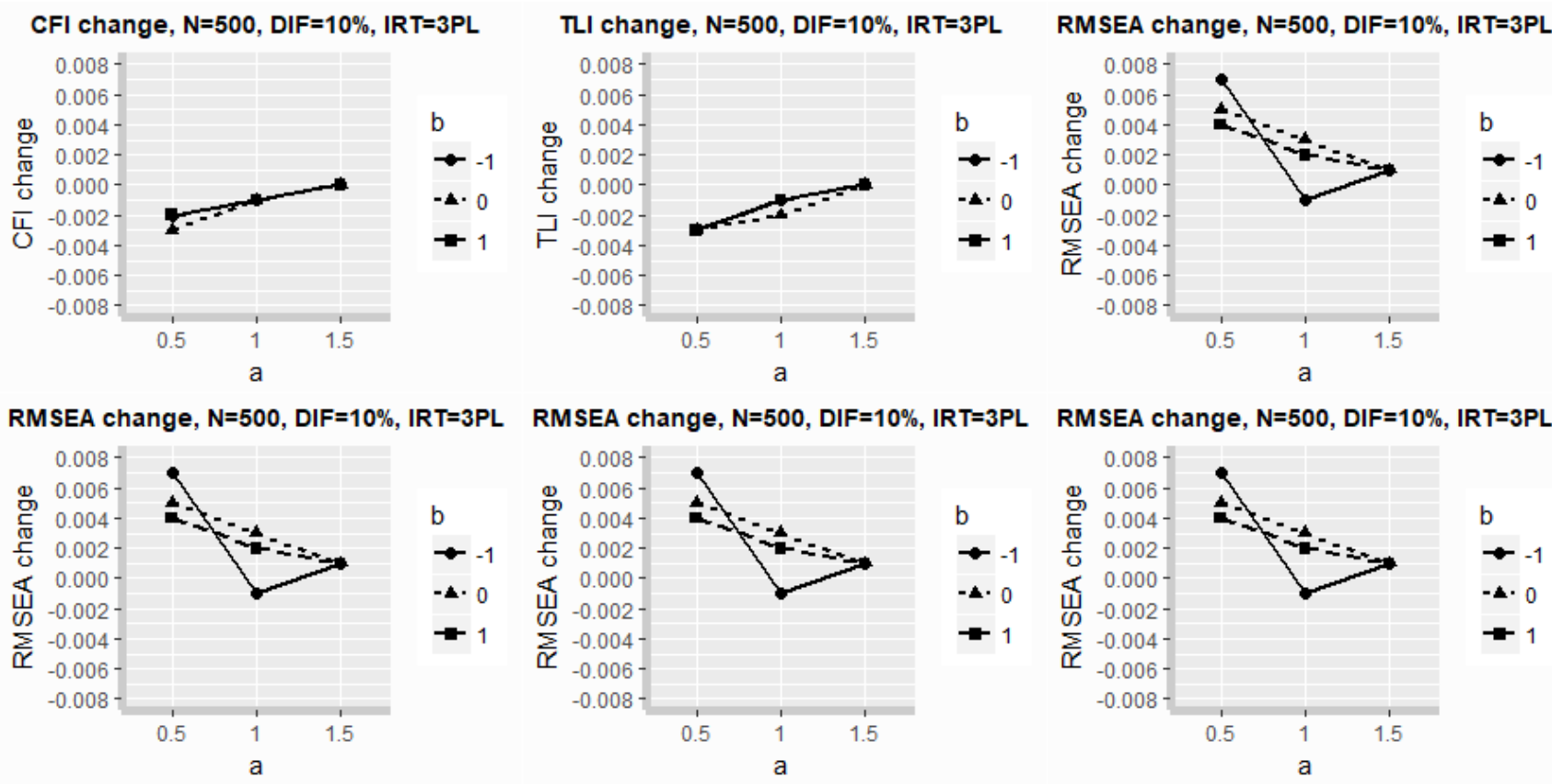

Figure 4.2. Change in equal loadings, equal threshold (scalar invariance) GFI's across conditions for $\mathrm{N}=500$, IRT=2PL.

Table 4.7 and Figure 4.3 show the results of the simulation when the sample size has increased to 1000 , and the IRT model is the 3PL. A close examination of this table confirms the effect of the $a$ parameter value on the magnitude of the GFI changes, especially on the TLI. The changes are more pronounced when the percentage of DIF is $10 \%$. When the percentage of DIF increases to $20 \%$, we can see that the small changes are consistent across conditions except for the TLI. One interesting pattern in these observations is the CFI, TLI, and the RMSEA changes at DIF $=20 \%, b=0$ and $a=1.5$. Only in this condition do the GFI's reflect the change associated with scalar invariance similar to the Cheung and Rensvold's (2002) recommended threshold of 0.01. Nevertheless, we can arrive at the same conclusion that none of the GFI's were able to capture the simulated scalar noninvariance, questioning the appropriateness of the cutoff value of 0.01 in current practice 
Table 4.7

Change in Equal Loadings Equal Threshold (Scalar Invariance) GFI's across conditions for $N=1000, I R T=3 P L$

\begin{tabular}{|c|c|c|c|c|c|c|c|c|c|c|}
\hline \multicolumn{2}{|c|}{ 3PL IRT } & \multicolumn{3}{|c|}{$b=-1$} & \multicolumn{3}{|c|}{$\mathbf{b}=\mathbf{0}$} & \multicolumn{3}{|c|}{$b=1$} \\
\hline & & $a=0.5$ & $\mathrm{a}=1.0$ & $\mathrm{a}=1.5$ & $\mathrm{a}=0.5$ & $a=1.0$ & $\mathrm{a}=1.5$ & $a=0.5$ & $\mathrm{a}=1.0$ & $a=1.5$ \\
\hline \multirow[t]{3}{*}{$10 \%$} & CFI & -0.001 & -0.000 & 0.000 & -0.001 & -0.000 & 0.000 & -0.000 & -0.000 & -0.000 \\
\hline & TLI & -0.005 & -0.002 & -0.001 & -0.005 & -0.003 & -0.001 & -0.004 & -0.001 & -0.000 \\
\hline & RMSEA & 0.001 & 0.000 & 0.000 & 0.001 & 0.000 & 0.000 & 0.001 & 0.000 & 0.000 \\
\hline \multirow[t]{3}{*}{$20 \%$} & CFI & -0.000 & 0.000 & 0.000 & -0.000 & 0.000 & -0.011 & -0.000 & 0.000 & 0.000 \\
\hline & TLI & -0.002 & -0.001 & 0.000 & -0.002 & -0.000 & -0.012 & -0.001 & 0.001 & 0.001 \\
\hline & RMSEA & 0.000 & 0.000 & 0.000 & 0.000 & 0.000 & 0.010 & 0.000 & 0.000 & -0.000 \\
\hline
\end{tabular}

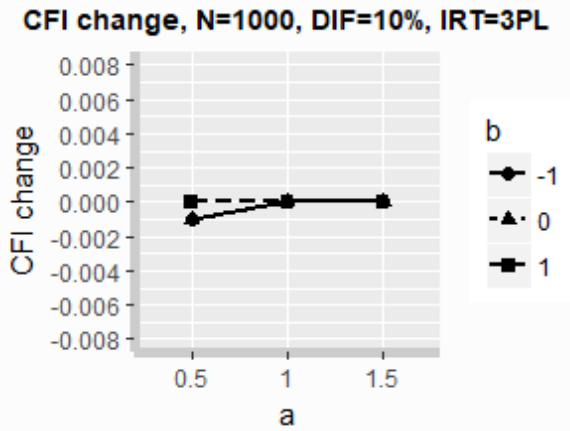

CFI change, $\mathrm{N}=1000, \mathrm{DIF}=20 \%$, IRT $=3 \mathrm{PL}$

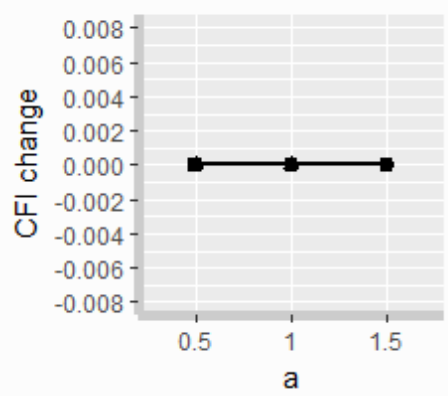

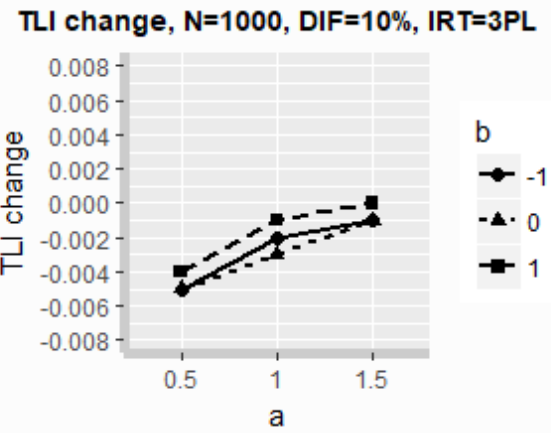

TLI change, $N=1000$, DIF=20\%, IRT=3PL

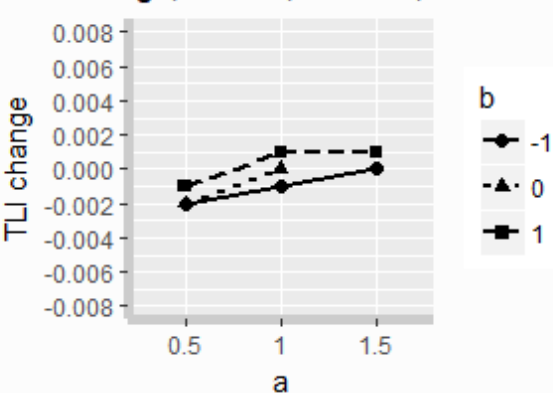

RMSEA change, $N=1000$, DIF $=10 \%$, IRT $=3 P L$

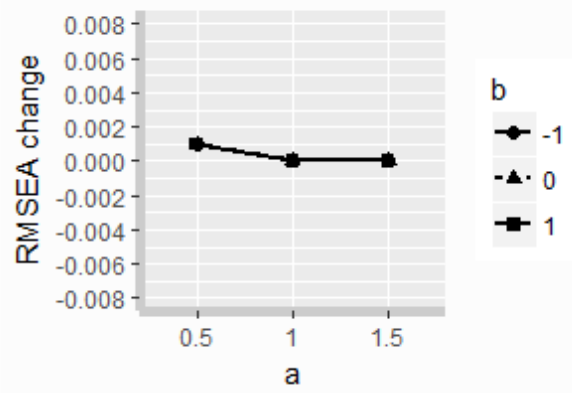

RMSEA change, $\mathrm{N}=1000$, DIF $=20 \%$, IR $\mathrm{T}=3 \mathrm{PL}$

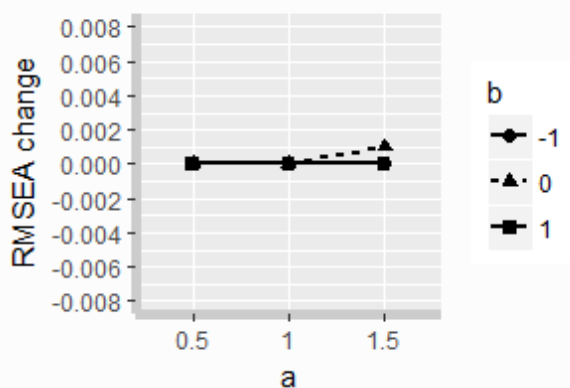

Figure 4.3. Change in equal loadings equal threshold (scalar invariance) GFI's across conditions for $\mathrm{N}=1000, \mathrm{IRT}=3 \mathrm{PL}$. 
Table 4.8 and Figure 4.4 present the simulation results for sample size 1000 and the 2PL IRT model. Overall, the GFI's fail to capture scalar noninvariance simulated in the data. The changes across conditions are very negligible and seem spurious if interpreted as scalar invariance. The role of the $a$ parameter value in decreasing change magnitude is noticeable across all conditions and the GFI's.

Table 4.8

Change in Equal Loadings Equal Threshold (Scalar Invariance) GFI's across conditions for $N=1000, I R T=2 P L$

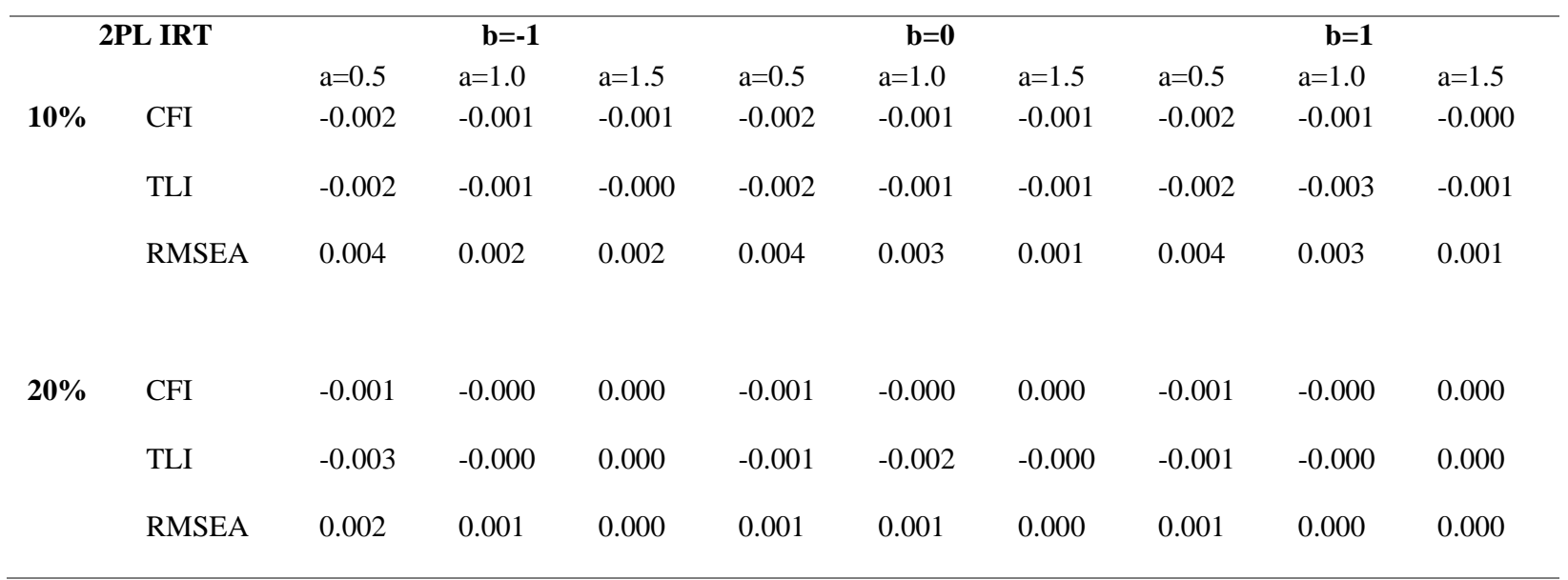




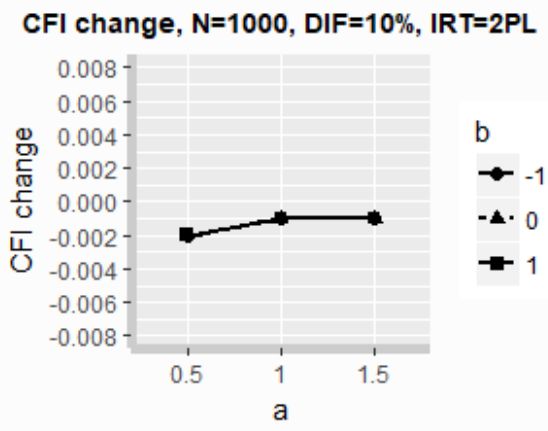

CFI change, $N=1000$, DIF $=20 \%, I R T=2 P L$

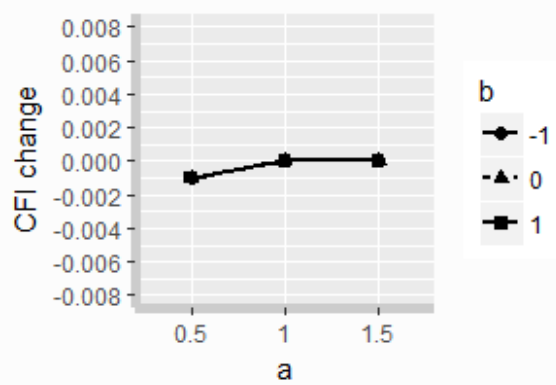

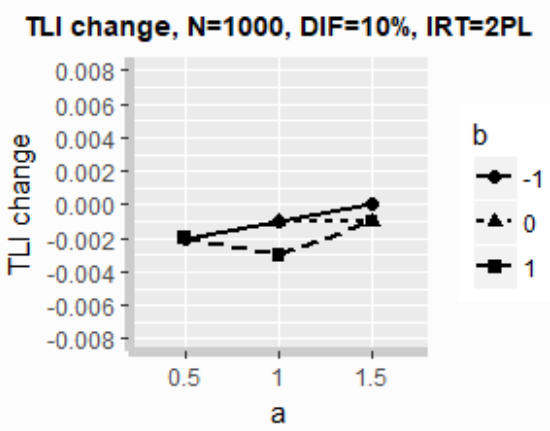

TLI change, $\mathrm{N}=1000$, DIF=20\%, IRT=2PL

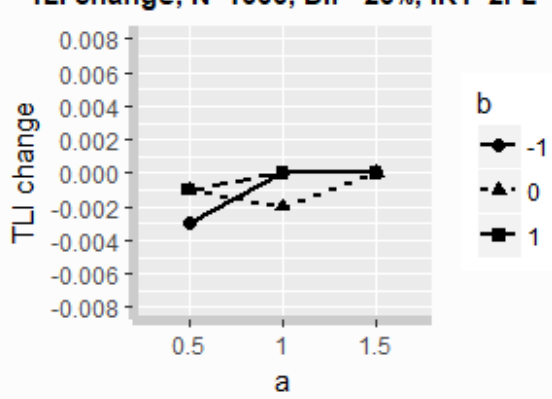

RMSEA change, $\mathrm{N}=1000$, DIF $=10 \%$, IR $T=2 \mathrm{PL}$

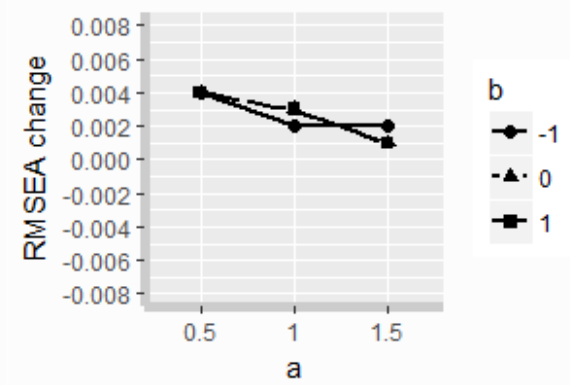

RMSEA change, $\mathrm{N}=1000$, DIF $=20 \%$, IR $\mathrm{T}=2 \mathrm{PL}$

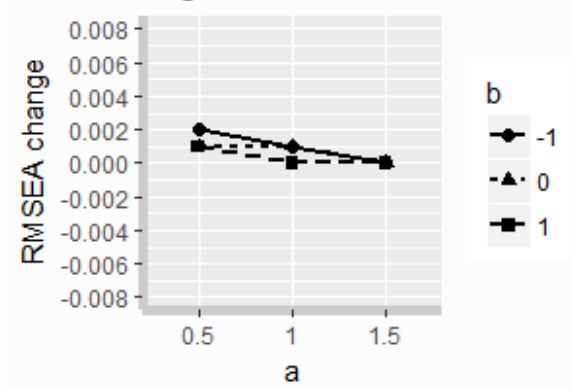

Figure 4.4. Change in equal loadings equal threshold (scalar invariance) GFI's across conditions for $\mathrm{N}=1000$, IRT=2PL.

Table 4.9 and Figure 4.5 exhibit the simulation results for sample size 2000 and the 3PL IRT model. Similar to the results in other sample sizes, the effect of the $a$ parameter on the changes in GFI's is present. The unrevealing changes in the GFI show that they have failed to capture the simulated scalar noninvariance in the data. 
Table 4.9

Change in Equal Loadings Equal Threshold (Scalar Invariance) GFI's across conditions for $N=2000, I R T=3 P L$

\begin{tabular}{|c|c|c|c|c|c|c|c|c|c|c|}
\hline \multirow{2}{*}{\multicolumn{2}{|c|}{ 3PL IRT }} & \multicolumn{3}{|c|}{$b=-1$} & \multicolumn{3}{|c|}{$\mathbf{b}=\mathbf{0}$} & \multicolumn{3}{|c|}{$b=1$} \\
\hline & & $a=0.5$ & $\mathrm{a}=1.0$ & $a=1.5$ & $\mathrm{a}=0.5$ & $\mathrm{a}=1.0$ & $a=1.5$ & $a=0.5$ & $\mathrm{a}=1.0$ & $a=1.5$ \\
\hline $10 \%$ & CFI & -0.003 & -0.001 & -0.00 & -0.003 & -0.001 & -0.000 & -0.002 & -0.001 & -0.000 \\
\hline & TLI & -0.006 & -0.003 & -0.002 & -0.006 & -0.004 & -0.002 & -0.004 & -0.002 & -0.001 \\
\hline & RMSEA & 0.004 & 0.002 & 0.001 & 0.004 & 0.002 & 0.001 & 0.002 & 0.001 & 0.001 \\
\hline
\end{tabular}

\begin{tabular}{llllllllllll}
\hline $\mathbf{2 0 \%}$ & CFI & -0.00 & -0.000 & -0.000 & -0.000 & -0.000 & -0.000 & -0.000 & -0.000 & 0.000 \\
\hline & TLI & -0.003 & -0.001 & -0.000 & -0.003 & -0.001 & -0.003 & -0.002 & -0.001 & 0.000 \\
& & & & & & & & & & & \\
\hline & RMSEA & 0.001 & 0.000 & 0.000 & 0.001 & 0.000 & 0.000 & 0.001 & 0.000 & -0.000
\end{tabular}

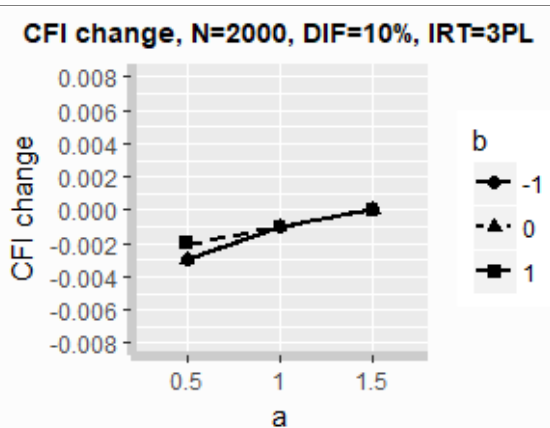

CFI change, $\mathrm{N}=2000$, DIF=20\%, IRT=3PL

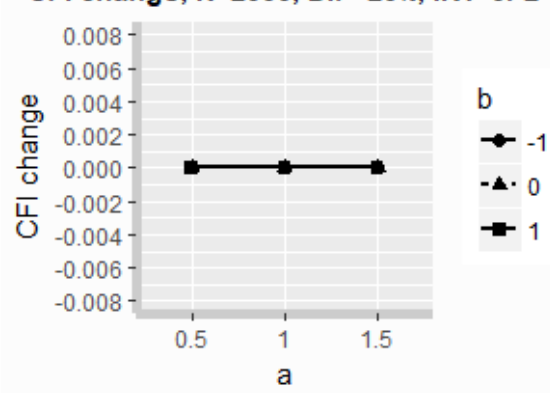

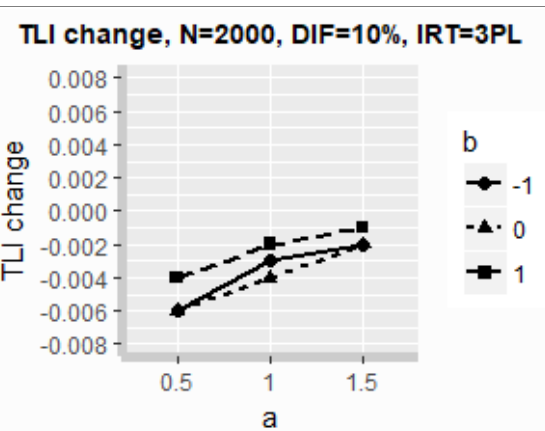

TLI change, $\mathrm{N}=2000, \mathrm{DIF}=20 \%$, IR $\mathrm{T}=3 \mathrm{PL}$

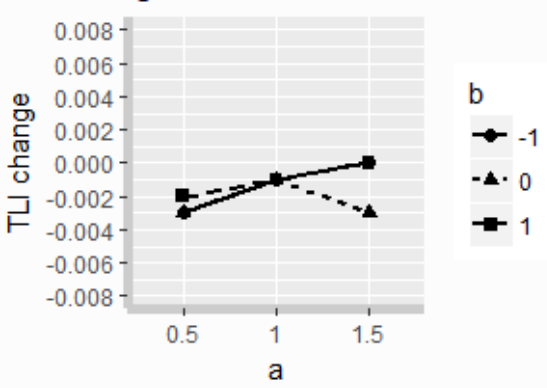

RMSEA change, $\mathrm{N}=2000$, DIF=10\%, IRT=3PL

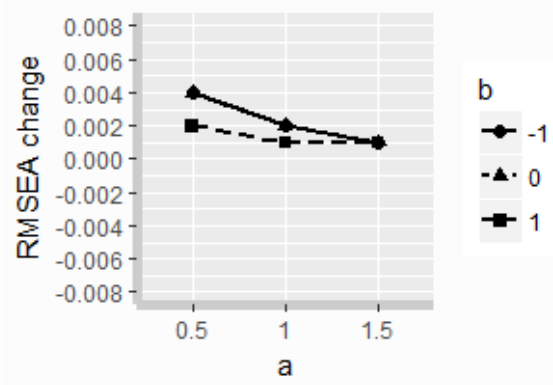

RMSEA change, $\mathrm{N}=2000$, DIF $=20 \%$, IRT=3PL

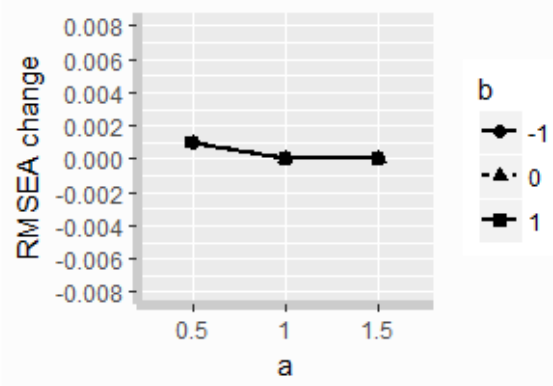

Figure 4.5. Change in equal loadings equal threshold (scalar invariance) GFI's across conditions for $\mathrm{N}=2000, \mathrm{IRT}=3 \mathrm{PL}$. 
Table 4.10 and Figure 4.6 present the simulation results when the sample size is 2000 and the IRT model is 2PL. We can see that in none of the conditions did the GFI's succeed in detecting simulated scalar noninvariance, implying that these indicators are not reliable when the consequence of measurement invariance has been preset.

Table 4.10

Change in Equal Loadings Equal Threshold (Scalar Invariance) GFI's across conditions for $N=2000, I R T=2 P L$

\begin{tabular}{|c|c|c|c|c|c|c|c|c|c|c|}
\hline \multirow{2}{*}{\multicolumn{2}{|c|}{ 2PL IRT }} & \multicolumn{3}{|c|}{$b=-1$} & \multicolumn{3}{|c|}{$b=0$} & \multicolumn{3}{|c|}{$b=1$} \\
\hline & & $\mathrm{a}=0.5$ & $\mathrm{a}=1.0$ & $\mathrm{a}=1.5$ & $a=0.5$ & $\mathrm{a}=1.0$ & $\mathrm{a}=1.5$ & $\mathrm{a}=0.5$ & $\mathrm{a}=1.0$ & $\mathrm{a}=1.5$ \\
\hline \multirow[t]{3}{*}{$10 \%$} & CFI & -0.003 & -0.002 & -0.001 & -0.003 & -0.000 & -0.001 & -0.003 & -0.000 & -0.001 \\
\hline & TLI & -0.002 & -0.001 & -0.001 & -0.003 & -0.002 & -0.001 & -0.003 & -0.001 & -0.001 \\
\hline & RMSEA & 0.006 & 0.004 & 0.003 & 0.007 & 0.000 & 0.003 & 0.007 & 0.000 & 0.003 \\
\hline
\end{tabular}

\begin{tabular}{rlllllllllll}
\hline $\mathbf{2 0 \%}$ & CFI & -0.000 & -0.000 & -0.000 & -0.000 & -0.000 & -0.000 & -0.001 & -0.000 & -0.000 \\
\hline & & & & & & & & & & \\
& TLI & -0.001 & -0.001 & -0.000 & -0.001 & -0.006 & -0.000 & -0.001 & -0.001 & -0.000 \\
\hline & & & & & & & & & & & \\
\hline
\end{tabular}


CFI change, $\mathrm{N}=\mathbf{2 0 0 0}$, DIF=10\%, IR $\mathrm{T}=2 \mathrm{PL}$

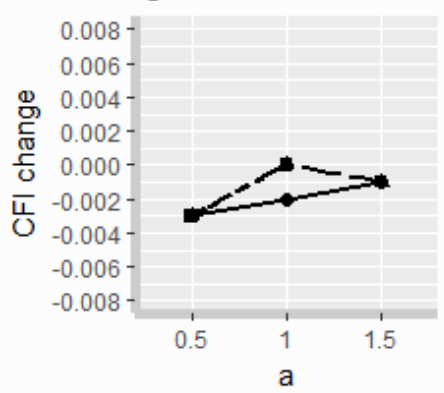

CFI change, $\mathrm{N}=\mathbf{2 0 0 0}$, DIF=20\%, IRT=2PL

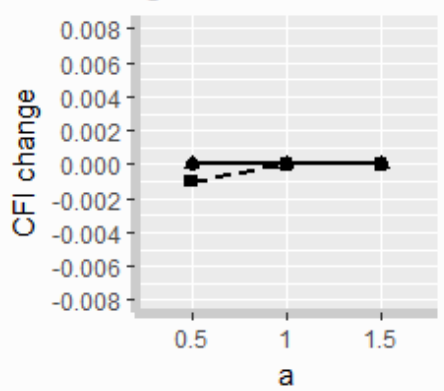

TLI change, $\mathrm{N}=2000$, DIF $=10 \%$, IRT $=2 \mathrm{PL}$
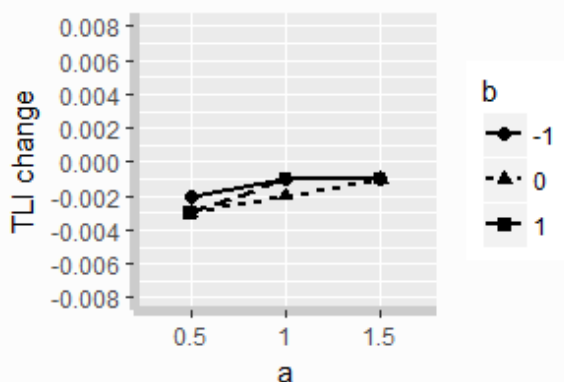

TLI change, $\mathrm{N}=\mathbf{2 0 0 0}$, DIF $=\mathbf{2 0} \%$, IRT $\mathrm{T}=2 \mathrm{PL}$

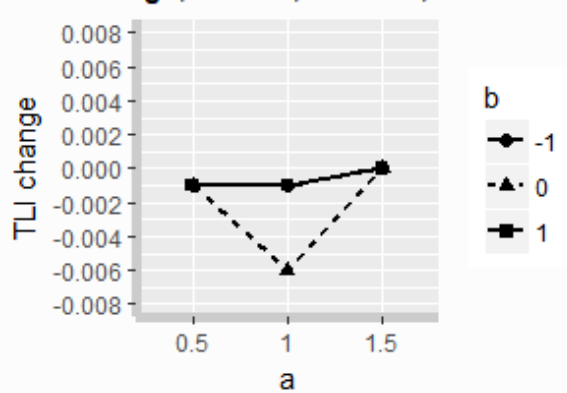

RMSEA change, $\mathrm{N}=\mathbf{2 0 0 0}$, DIF=10\%, IRT=2PL

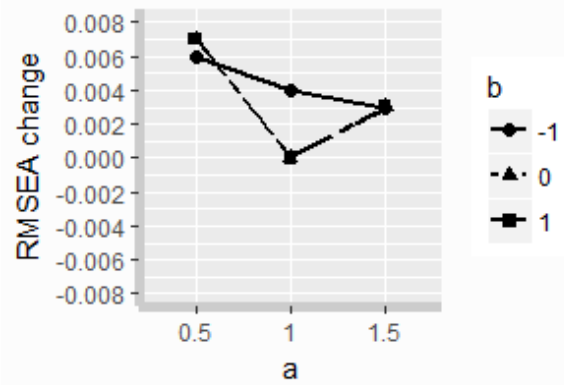

RMSEA change, $\mathrm{N}=\mathbf{2 0 0 0}$, DIF $=\mathbf{2 0} \%$, IR $\mathrm{T}=\mathbf{2 P L}$

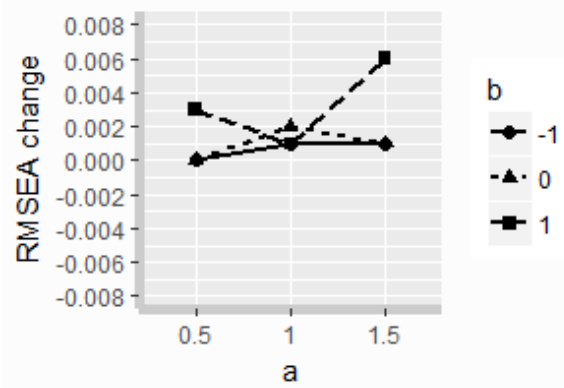

Figure 4.6. Change in equal loadings equal threshold (scalar invariance) GFI's across conditions for $\mathrm{N}=2000$, IRT=2PL.

The other research question in the study concerned the distribution of the changes in the fit indices (the CFI, the TLI, and the RMSEA). Standard deviation, skewedness, and the kurtosis of the changes in fit indices were calculated for each study condition to address this question. Tables 4.11-4.16 and Figures 4.7 through 4.18 show the dispersion measure values of the change in fit indices in tabular and graphical forms. 
Table 4.11

Distribution statistics Standard Deviation (SD), Skewedness (SK), and Kurtosis (KU) for change in CFI, TLI, and RMSEA at scalar level across conditions for $N=500, I R T=3 P L$.

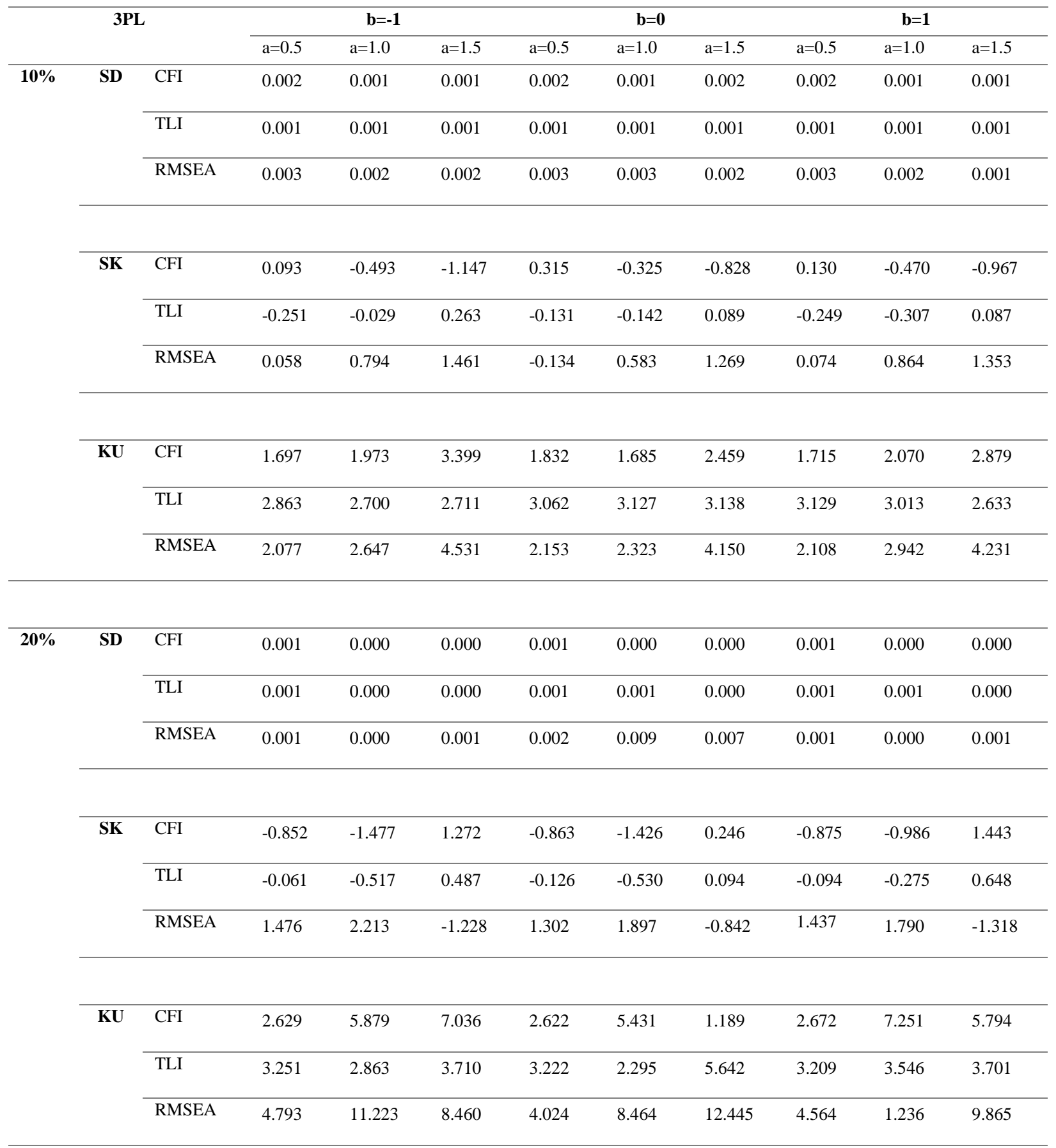




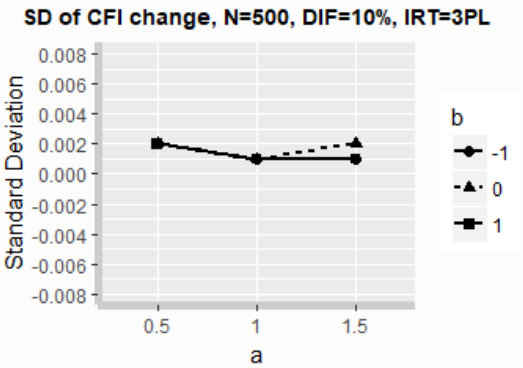

SK of $C F I$ change, $N=500$, DIF $=10 \%, I R T=3 P L$

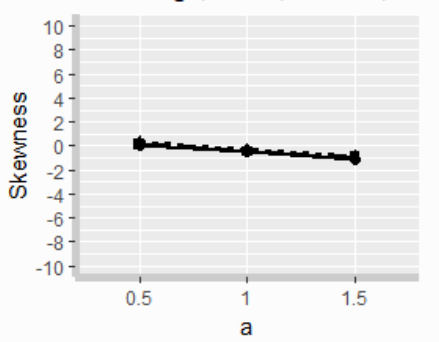

$\mathrm{KU}$ of $\mathrm{CFI}$ change, $\mathrm{N}=500$, DIF $=10 \%$, IRT $=3 \mathrm{PL}$

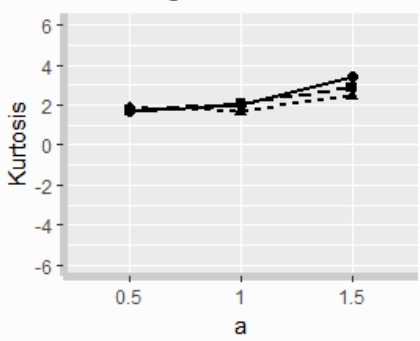

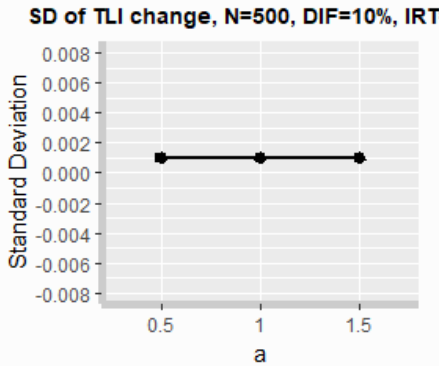

SK of TLI change, $N=500$, DIF=10\%, IRT=3PL

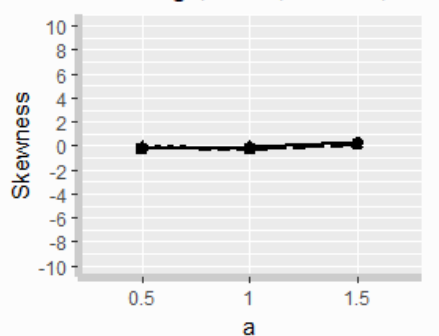

$\mathrm{KU}$ of $\mathrm{TLI}$ change, $\mathrm{N}=500$, DIF $=10 \%$, IRT=3PL

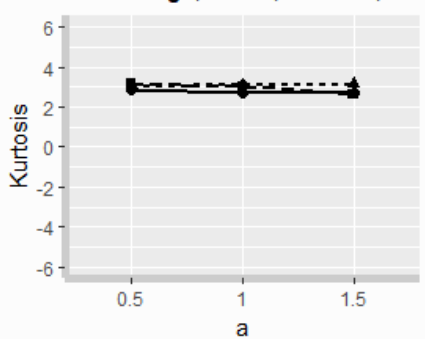

SD of RMSEA change, $N=500$, DIF $=10 \%$, IRT $=3 P L$

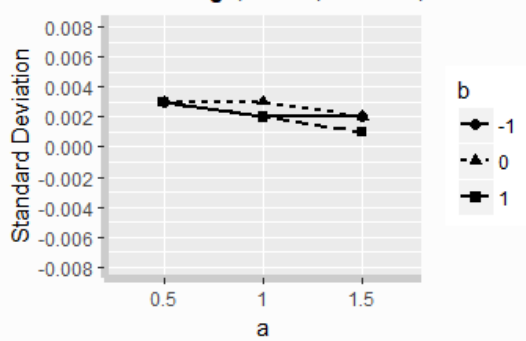

SK of RMSEA change, N=500, DIF=10\%, IRT=3PL

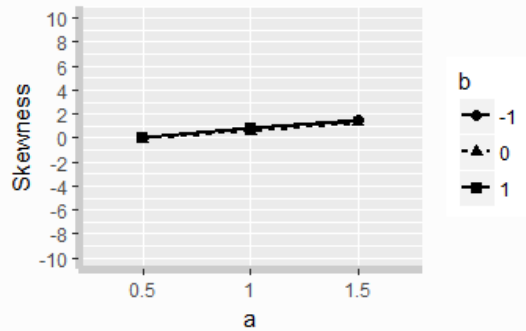

KU of RMSEA change, N=500, DIF $=10 \%, I R T=3 P L$

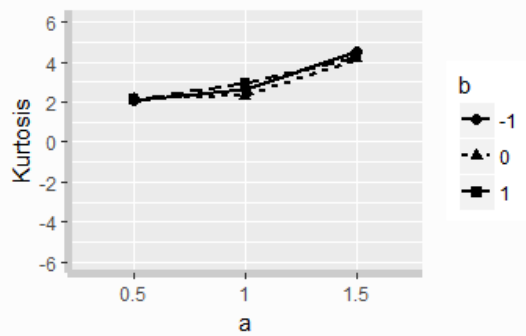

Figure 4.7. Dispersion statistics ( $\mathrm{SD}=$ standard deviation, $\mathrm{SK}=$ skewedness, $\mathrm{KU}=$ kurtosis) for the distributions of the change in the CFI, TLI, and the RMSEA fit indices at scalar level across simulation conditions for $\mathrm{N}=500, \mathrm{IRT}=3 \mathrm{PL}$, DIF percentage $=10 \%$ 


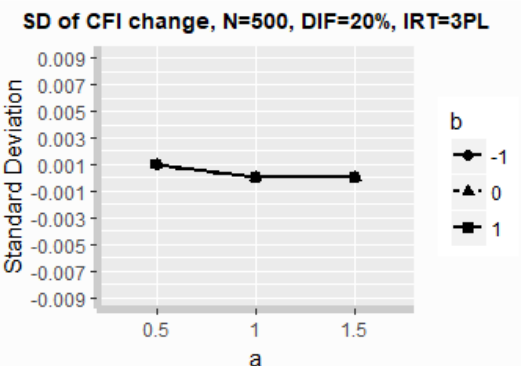

SK of CFI change, $N=500$, DIF $=20 \%$, IRT=3PL

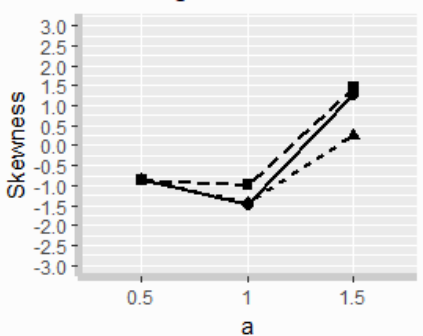

KU of CFI change, $\mathrm{N}=500$, DIF $=20 \%, I R T=3 P L$

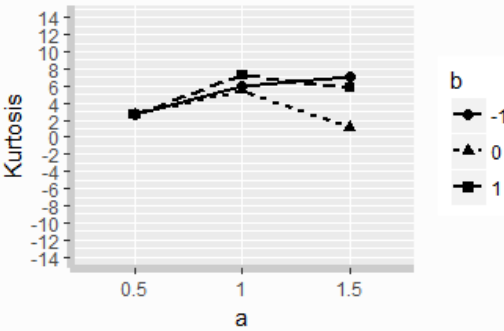

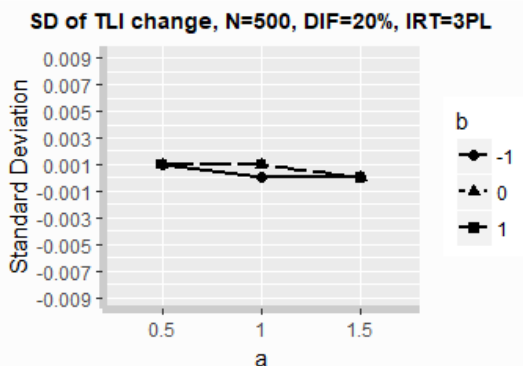

SK of TLI change, N=500, DIF= $20 \%, I R T=3 P L$
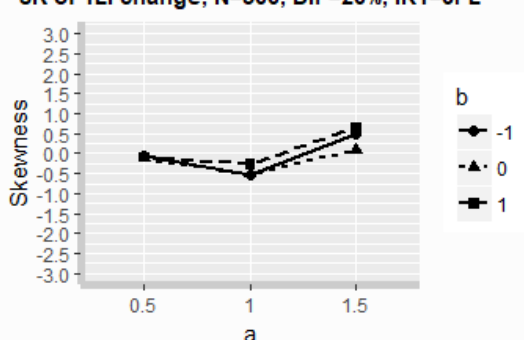

$\mathrm{KU}$ of $\mathrm{TLI}$ change, $\mathrm{N}=500$, DIF=20\%, IRT=3PL

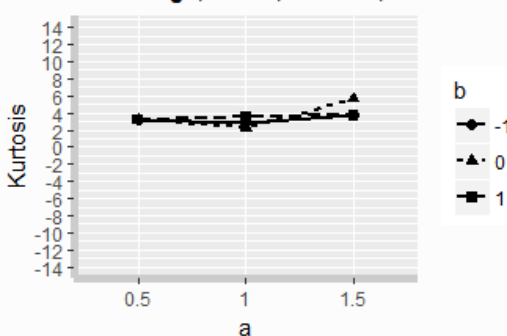

SD of RMSEA change, $\mathrm{N}=500$, DIF $=20 \%$, IRT=3PL

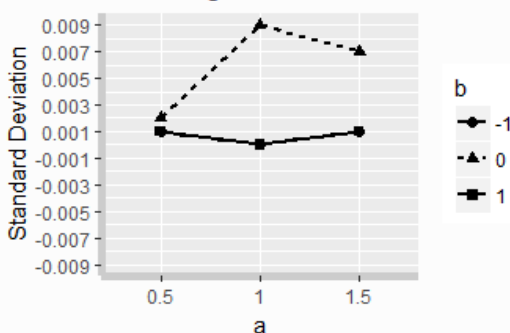

SK of RMSEA change, $\mathrm{N}=500$, DIF $=20 \%$, IRT $=3 \mathrm{PL}$

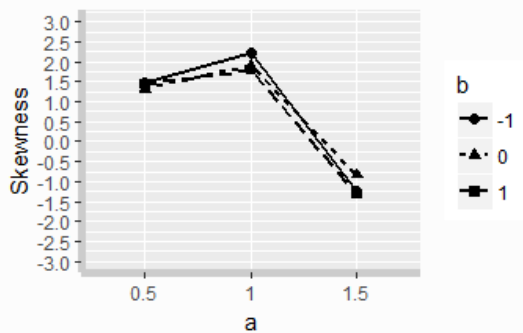

KU of RMSEA change, N=500, DIF $=20 \%$, IRT $=3 \mathrm{PL}$

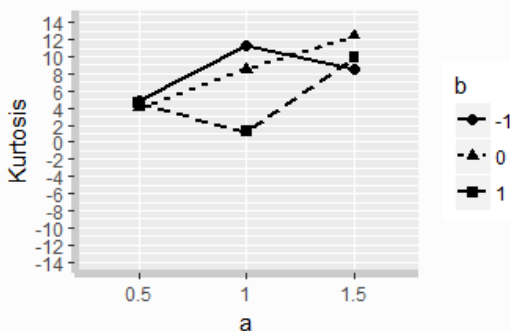

Figure 4.8. Dispersion statistics ( $\mathrm{SD}=$ standard deviation, $\mathrm{SK}=$ skewedness, $\mathrm{KU}=$ kurtosis) for the distributions of the change in the CFI, TLI, and the RMSEA fit indices at scalar level across simulation conditions for $\mathrm{N}=500, \mathrm{IRT}=3 \mathrm{PL}$, DIF percentage $=20 \%$

As Table 4.11 and Figures 4.7 and 4.8 above show, the standard deviation values are close to zero, implying that the estimated values for the change in fit indices are consistent. The absolute value of the skewness of the distributions for the CFI increases as the $a$ parameter increases. The same pattern can be seen (yet less consistently) for the TLI. The skewness of the RMSEA increases from $\mathrm{a}=0.5$ to $\mathrm{a}=1.0$, but then decreases and changes direction. The kurtosis value for the CFI is erratic, while that of TLI is more consistent and similar to normal distribution. The kurtosis for the RMSEA increases as the $a$ parameter increases. 
Table 4.12

Distribution statistics Standard Deviation (SD), Skewedness (SK), and Kurtosis (KU) for change in CFI, TLI, and RMSEA at scalar level across conditions for $N=500, I R T=2 P L$.

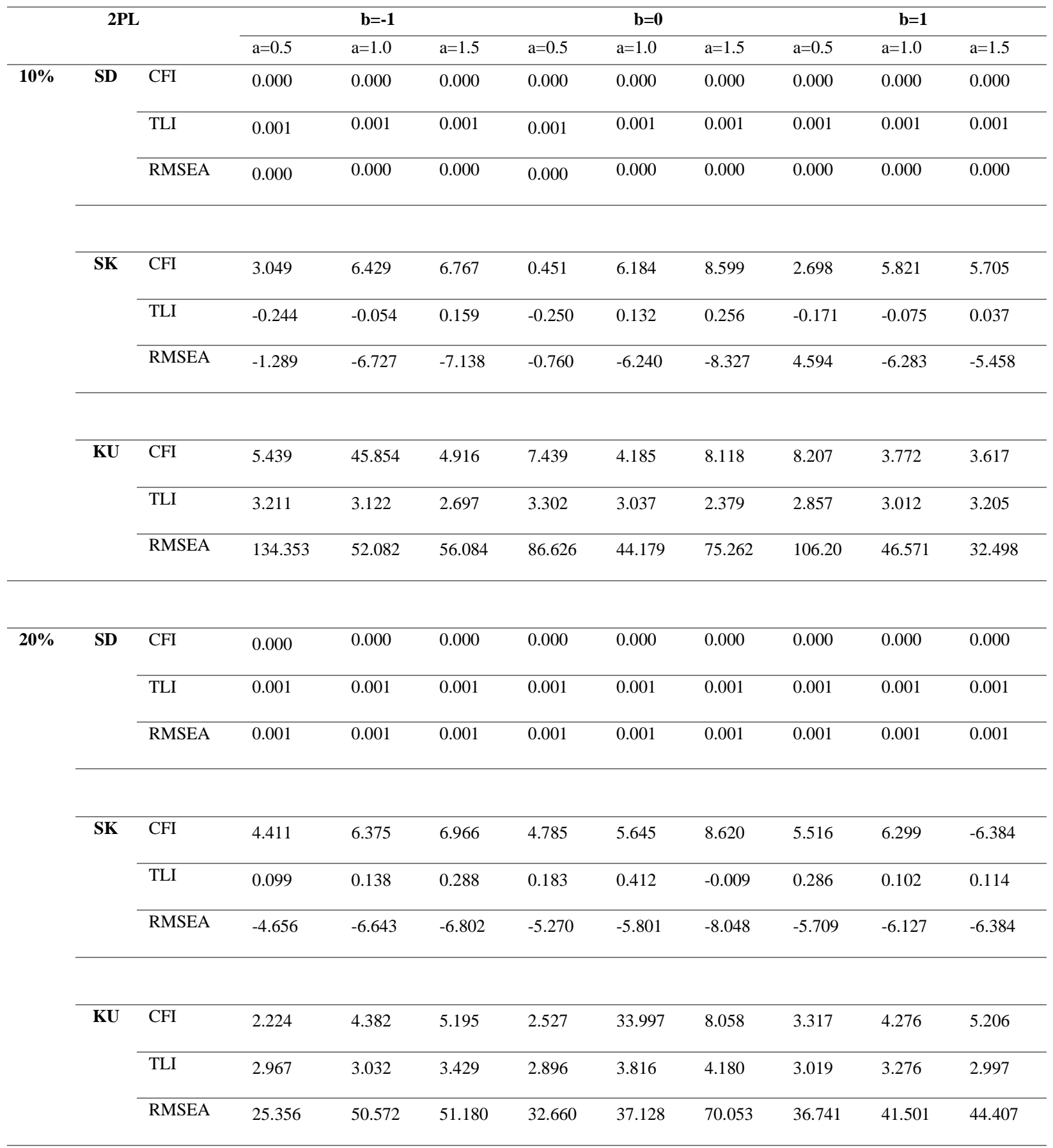


SD of CFI change, $N=500$, DIF $=10 \%, I R T=2 P L$

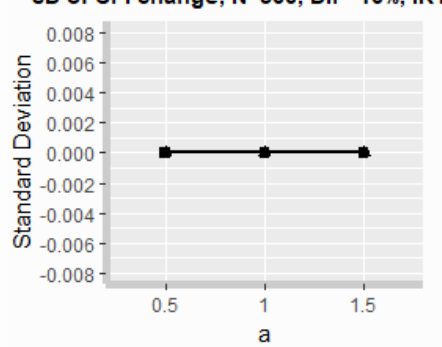

SK of $\mathrm{CFI}$ change, $\mathrm{N}=500, \mathrm{DIF}=10 \%, \mathrm{IRT}=2 \mathrm{PL}$

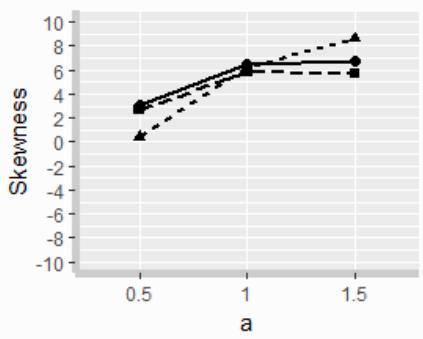

KU of CFI change, $N=500$, DIF $=10 \%, I R T=2 P L$

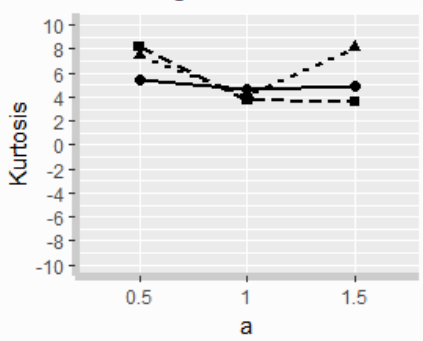

SD of $T L I$ change, $N=500$, DIF $=10 \%, I R T=2 P L$

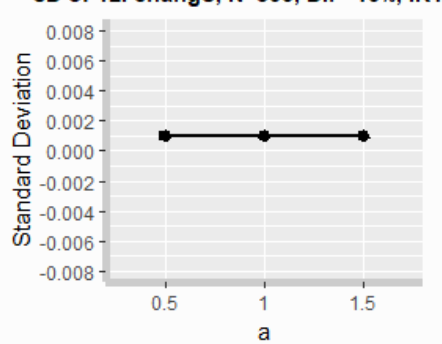

SK of $\mathrm{TLI}$ change, $\mathrm{N}=500$, DIF=10\%, IRT=2PL

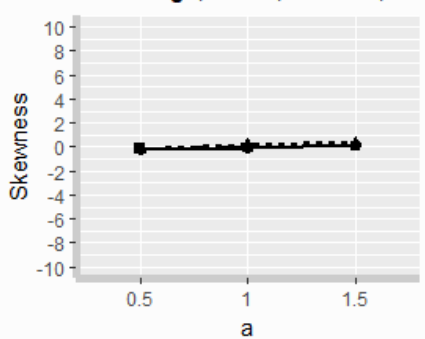

KU of TLI change, $N=500$, DIF $=10 \%, I R T=2 P L$

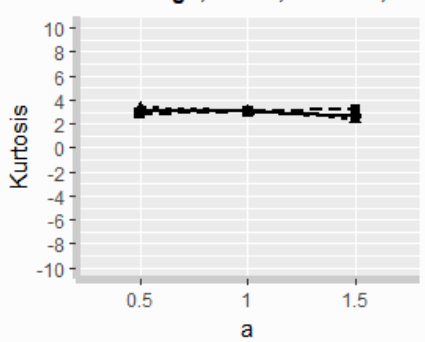

SD of RMSEA change, $N=500$, DIF $=10 \%$, IRT $=2 P L$

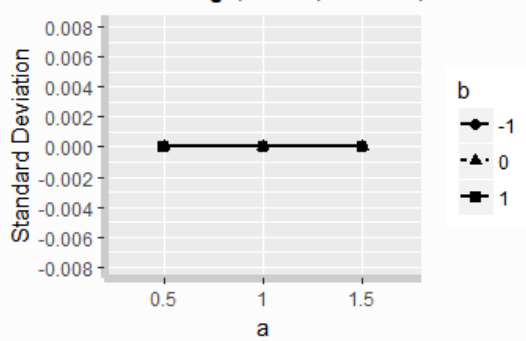

SK of RMSEA change, N=500, DIF $=10 \%$, IRT $=2 P L$

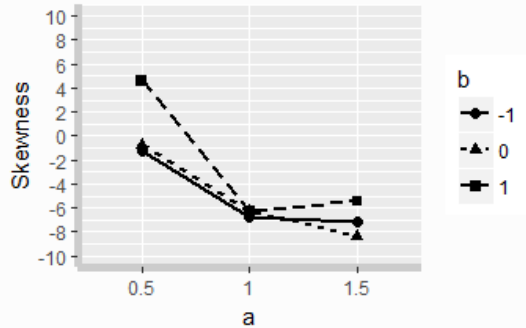

KU of RMSEA change, $N=500, D I F=10 \%, I R T=2 P L$

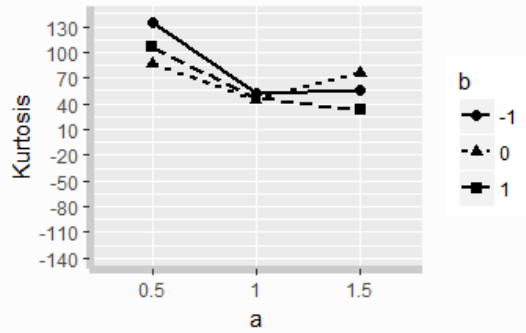

Figure 4.9. Dispersion statistics ( $\mathrm{SD}=$ standard deviation, $\mathrm{SK}=$ skewedness, $\mathrm{KU}=$ kurtosis) for the distributions of the change in the CFI, TLI, and the RMSEA fit indices at scalar level across simulation conditions for $\mathrm{N}=500, \mathrm{IRT}=2 \mathrm{PL}$, DIF percentage $=10 \%$ 

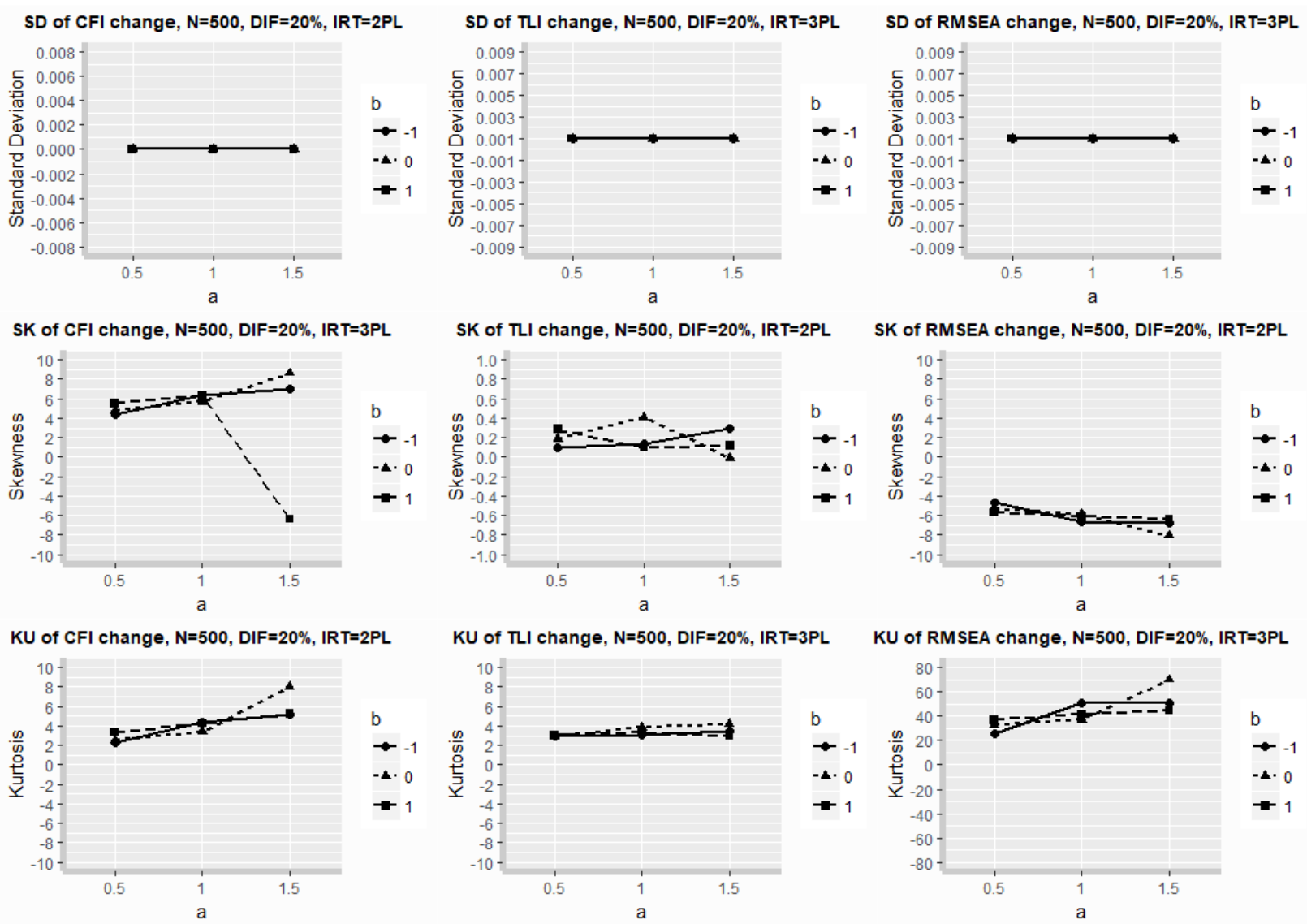

Figure 4.10. Dispersion statistics ( $\mathrm{SD}=$ standard deviation, $\mathrm{SK}=$ skewedness, $\mathrm{KU}=$ kurtosis) for the distributions of the change in the CFI, TLI, and the RMSEA fit indices at scalar level across simulation conditions for $\mathrm{N}=500, \mathrm{IRT}=2 \mathrm{PL}$, DIF percentage $=20 \%$

As Table 4.12 and Figures 4.9 and 4.10 above show, the standard deviation values are close to zero, implying that the estimated values for the change in fit indices across the replications are very consistent and also close to the mean. The absolute value of the skewness of the distributions for the CFI increases as the $a$ parameter increases. Skewness is smallest for the TLI distribution, compared with the distributions of the CFI and the RMSEA. The kurtosis values for TLI distribution are close to the normal, but for the RMSEA are far from the normal. For the CFI, they are also far from the normal (but less severely compared to the RMSEA). 
Table 4.13

Distribution statistics Standard Deviation (SD), Skewedness (SK), and Kurtosis (KU) for change in CFI, TLI, and RMSEA at scalar level across conditions for $N=1000, I R T=3 P L$.

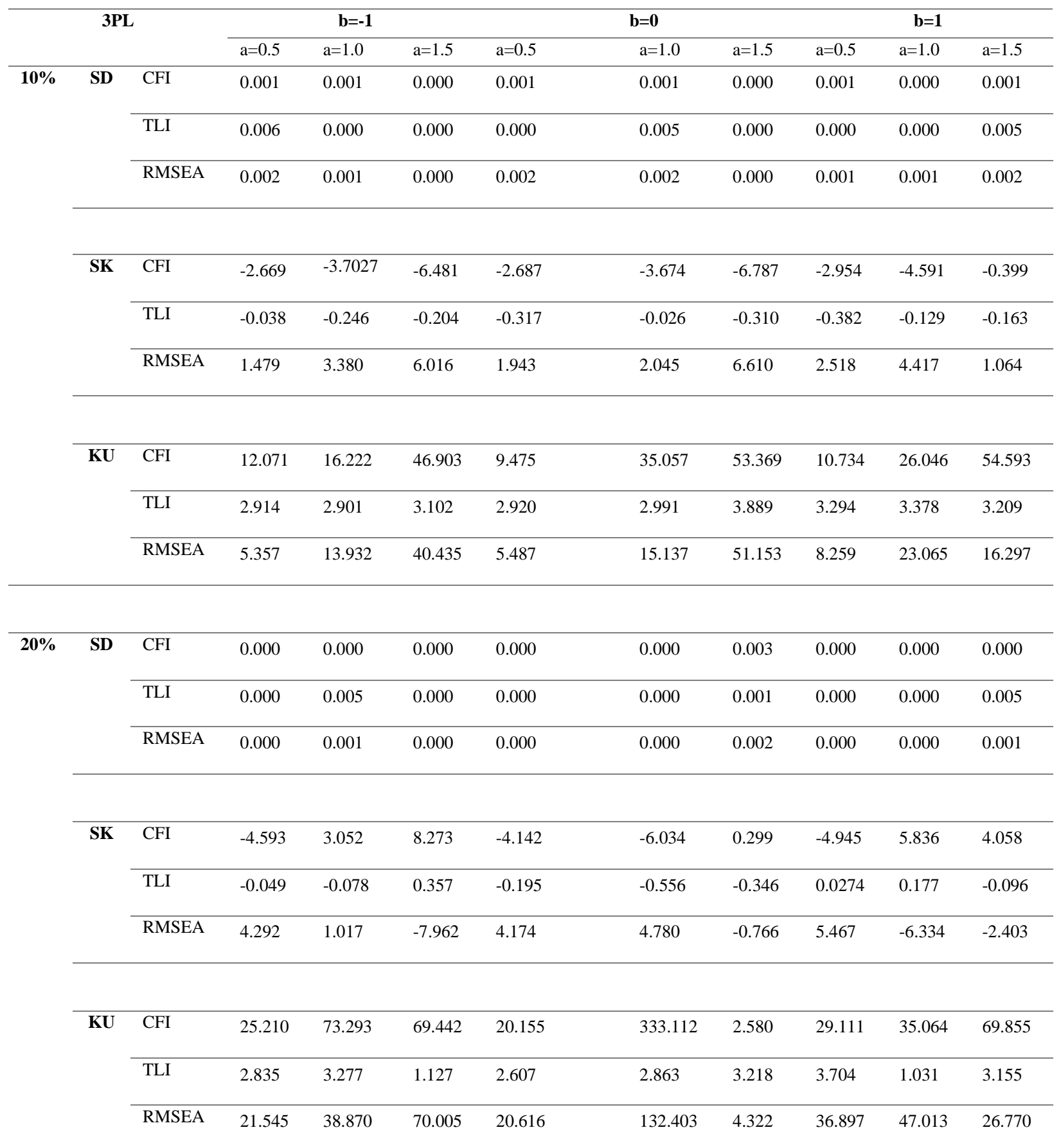


SD of $C F I$ change, $N=1000$, DIF $=10 \%$, IRT=3PL

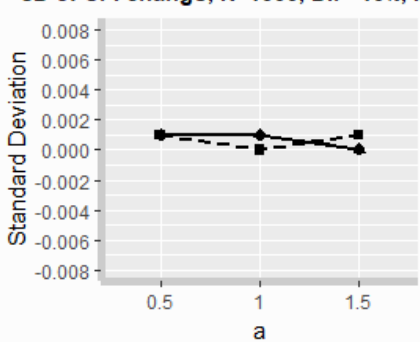

SK of CFI change, $N=1000, D I F=10 \%$, IRT $=3 P L$

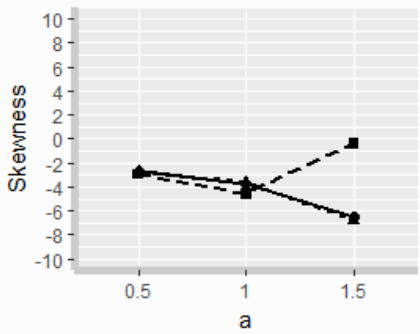

KU of CFI change, $N=1000$, DIF $=10 \%$, IRT=3PL

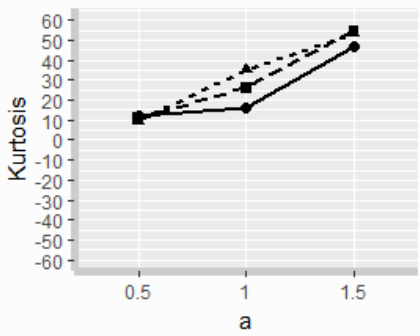

SD of $\mathrm{TLI}$ change, $\mathrm{N}=1000$, DIF $=10 \%$, IRT $=3 \mathrm{PL}$

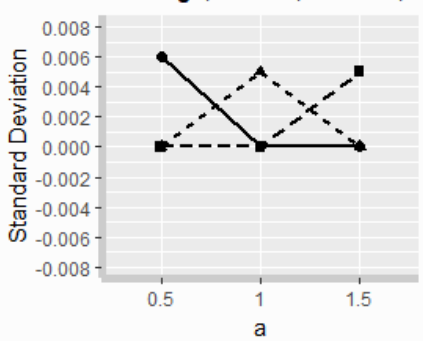

SK of TLI change, $N=1000$, DIF $=10 \%, I R T=3 P L$
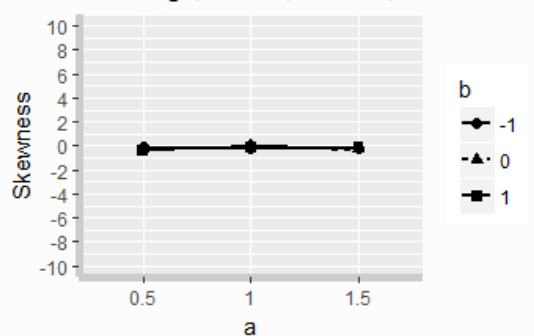

$\mathrm{KU}$ of $\mathrm{TLI}$ change, $\mathrm{N}=1000$, DIF $=10 \%, \mathrm{IRT}=3 \mathrm{PL}$

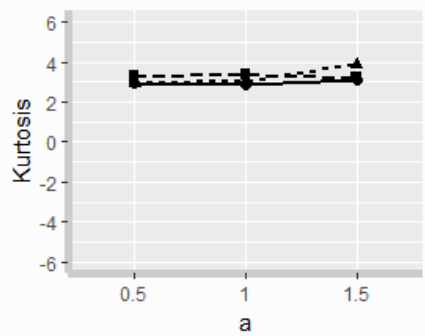

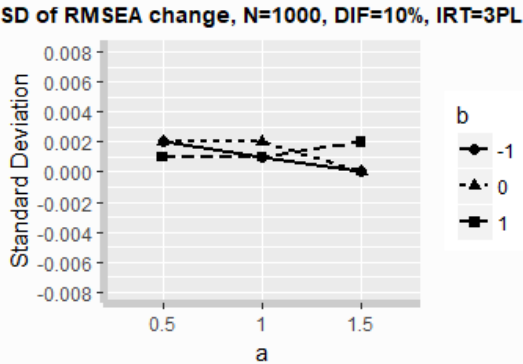

SK of RMSEA change, $N=1000$, DIF $=10 \%$, IRT $=3 P L$

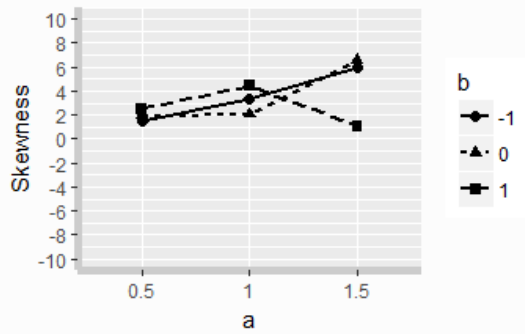

KU of RMSEA change, $\mathrm{N}=1000$, DIF $=10 \%$, IRT=3PL

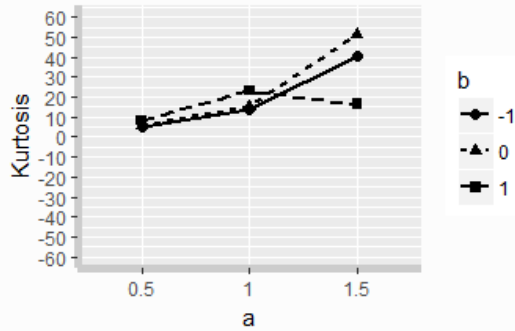

Figure 4.11. Dispersion statistics $(\mathrm{SD}=$ standard deviation, $\mathrm{SK}=$ skewedness, $\mathrm{KU}=$ kurtosis) for the distributions of the change in the CFI, TLI, and the RMSEA fit indices at scalar level across simulation conditions for $\mathrm{N}=1000, \mathrm{IRT}=3 \mathrm{PL}$, DIF percentage $=10 \%$ 

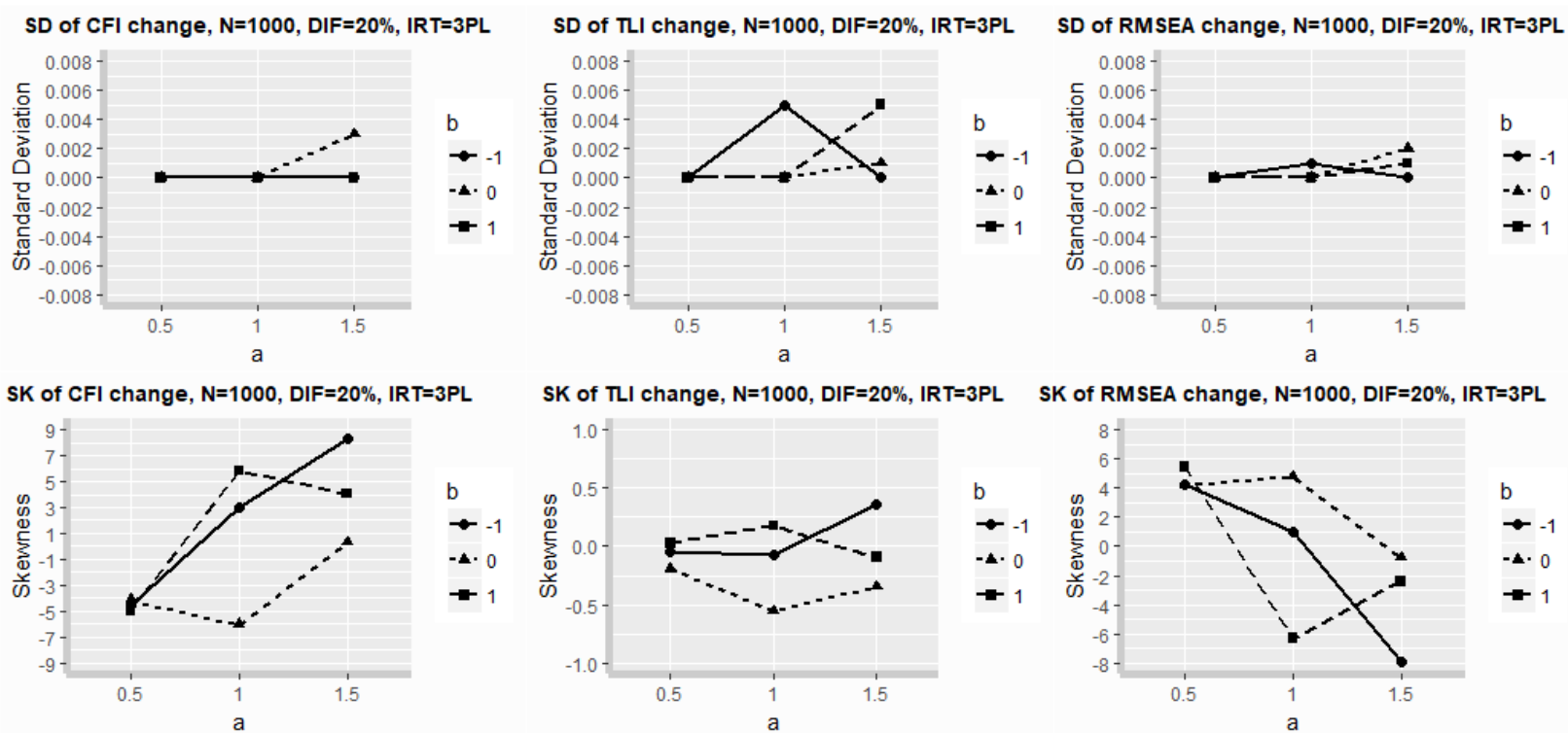

$\mathrm{KU}$ of $\mathrm{CFI}$ change, $\mathrm{N}=1000, \mathrm{DIF}=20 \%, \mathrm{IRT}=3 \mathrm{PL}$

$\mathrm{KU}$ of $\mathrm{TLI}$ change, $\mathrm{N}=1000, \mathrm{DIF}=20 \%, \mathrm{IRT}=3 \mathrm{PL}$

KU of RMSEA change, N=1000, DIF $=20 \%$, IRT=3PL
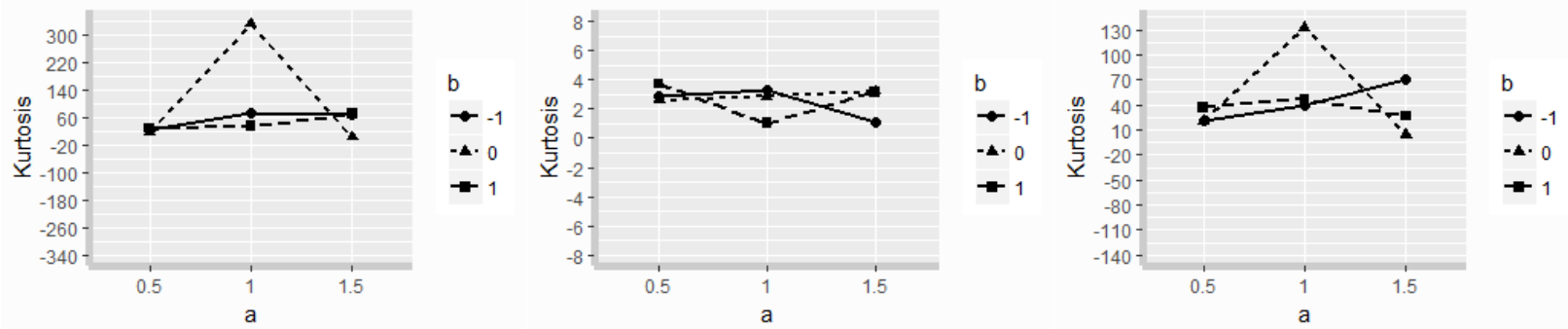

Figure 4.12. Dispersion statistics ( $\mathrm{SD}=$ standard deviation, $\mathrm{SK}=$ skewness, $\mathrm{KU}=$ kurtosis) for the distributions of the change in the CFI, TLI, and the RMSEA fit indices at scalar level across simulation conditions for $\mathrm{N}=1000, \mathrm{IRT}=3 \mathrm{PL}$, DIF percentage $=20 \%$

As Table 4.13 and Figures 4.11 and 4.12 above show, the standard deviation values for the CFI, the TLI, and RMSEA fit indices are close to zero, implying that the estimated values for the change in fit indices are very consistent. The absolute value of skewness for the CFI shows deviation from the normal distribution. The same holds for the RMSEA index, while that of the TLI remains close to the normal range. However, there is variation across the conditions in all of Othe fit indices. The kurtosis values are far from the normal for the CFI and the RMSEA. In addition, noticeable variation can be observed across the study conditions. For instance, the kurtosis increases as the $a$ parameter increases. 
Table 4.14

Distribution statistics Standard Deviation (SD), Skewness (SK), and Kurtosis (KU) for change in CFI, TLI, and RMSEA at scalar level across conditions for $N=1000, I R T=2 P L$.

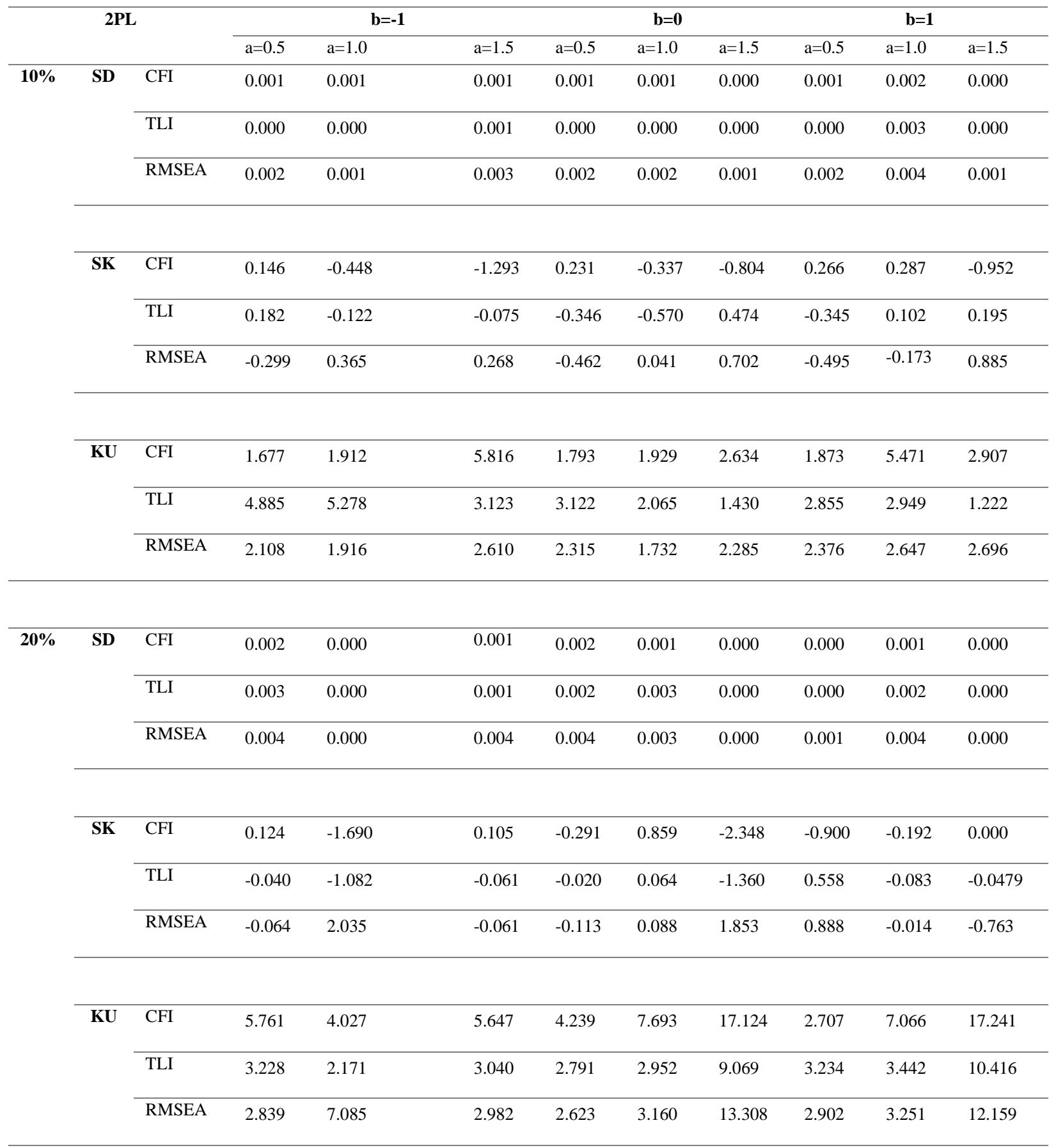


SD of $C F I$ change, $N=1000$, DIF $=10 \%$, IRT=2PL

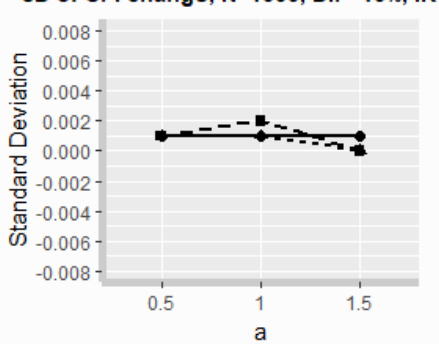

SK of CFI change, $N=1000, \mathrm{DIF}=10 \%, \mathrm{IRT}=2 \mathrm{PL}$

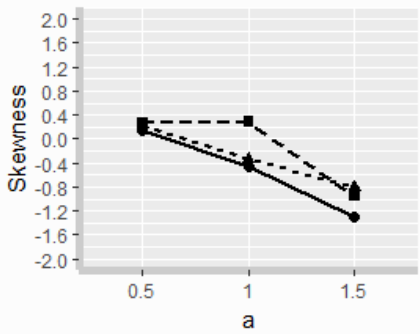

$\mathrm{KU}$ of $\mathrm{CFI}$ change, $\mathrm{N}=1000$, DIF $=10 \%$, IRT=2PL

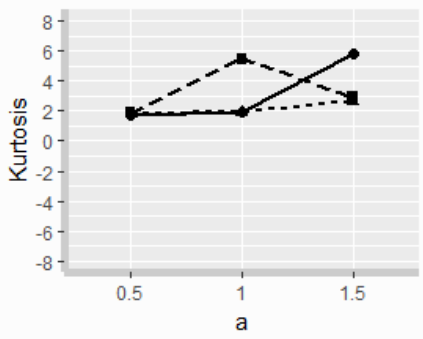

SD of $\mathrm{TLI}$ change, $\mathrm{N}=1000$, DIF=10\%, IRT=2PL

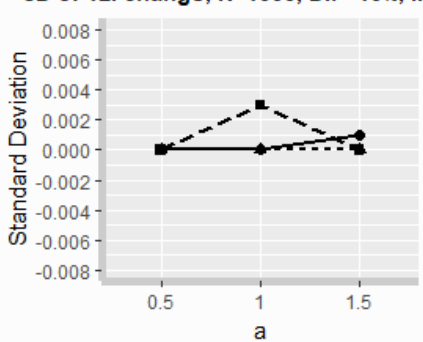

SK of TLI change, $N=1000$, DIF $=10 \%, I R T=2 P L$
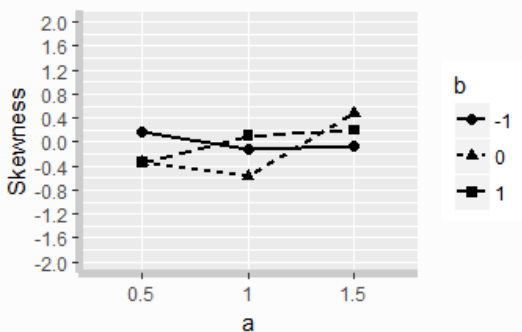

$\mathrm{KU}$ of $\mathrm{TLI}$ change, $\mathrm{N}=1000, \mathrm{DIF}=10 \%, \mathrm{IRT}=2 \mathrm{PL}$

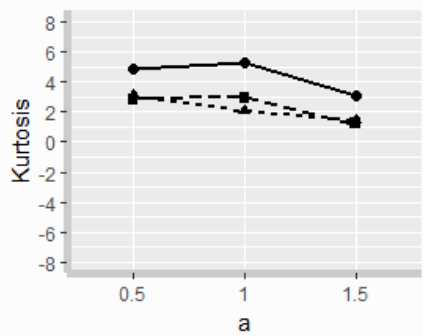

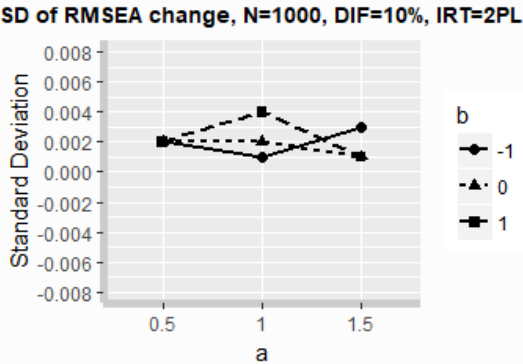

SK of RMSEA change, N=1000, DIF $=10 \%$, IRT $=2 P L$

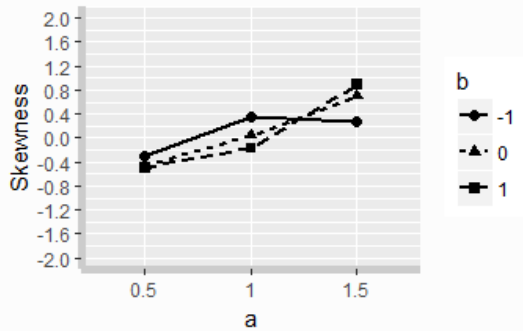

KU of RMSEA change, $N=1000$, DIF $=10 \%, I R T=2 P L$

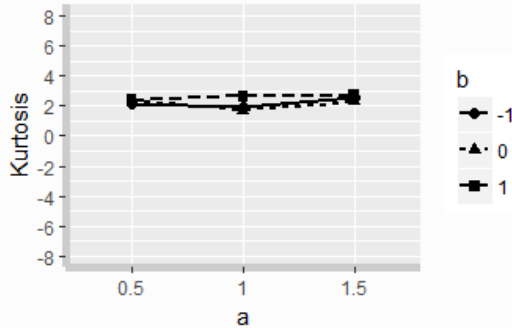

Figure 4.13. Dispersion statistics ( $\mathrm{SD}=$ standard deviation, $\mathrm{SK}=$ skewness, $\mathrm{KU}=$ kurtosis) for the distributions of the change in the CFI, TLI, and the RMSEA fit indices at scalar level across simulation conditions for $\mathrm{N}=1000, \mathrm{IRT}=2 \mathrm{PL}$, DIF percentage $=10 \%$ 

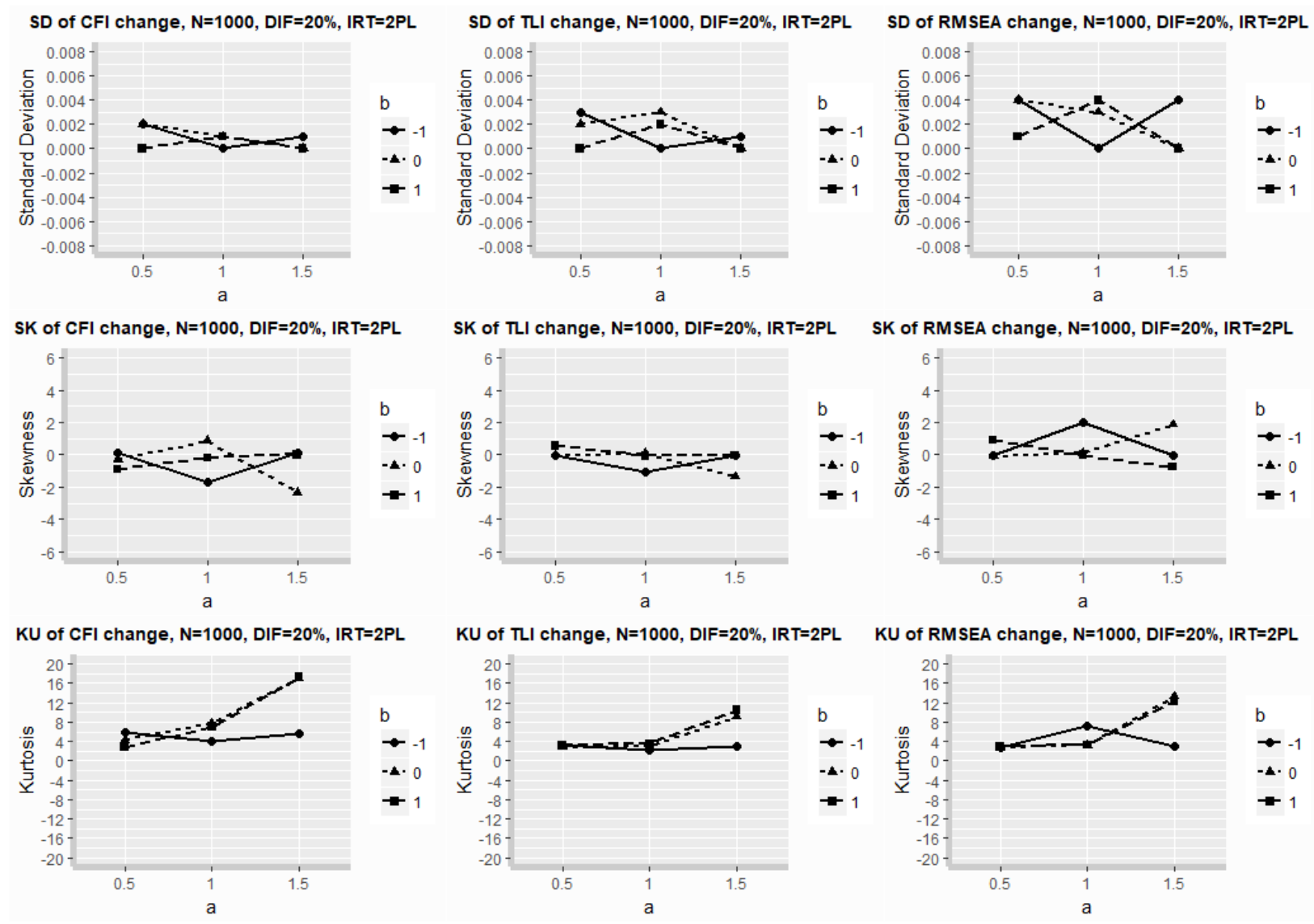

Figure 4.14. Dispersion statistics ( $\mathrm{SD}=$ standard deviation, $\mathrm{SK}=$ skewness, $\mathrm{KU}=$ kurtosis) for the distributions of the change in the CFI, TLI, and the RMSEA fit indices at scalar level across simulation conditions for $\mathrm{N}=1000, \mathrm{IRT}=2 \mathrm{PL}, \mathrm{DIF}$ percentage $=20 \%$

Similar to the statistics for sample size 500, as Table 4.14 and Figure 4.13 and 4.14 above show, the standard deviation values for the CFI, the TLI, and RMSEA fit indices are close to zero, implying that the estimated values for the change in fit indices across the replications are very consistent, close to the normal. The absolute value of skewness for the fit indices show minor to mild deviation from symmetry. The kurtosis is close to the normal range, but increasing for the CFI, decreasing for the TLI, and almost consistent for the RMSEA across the $a$ values. When the percentage of DIF increases to 20\%, the kurtosis increases for the CFI measure. 
Table 4.15

Distribution statistics Standard Deviation (SD), Skewness (SK), and Kurtosis (KU) for change in CFI, TLI, and RMSEA at scalar level across conditions for $N=2000, I R T=3 P L$.

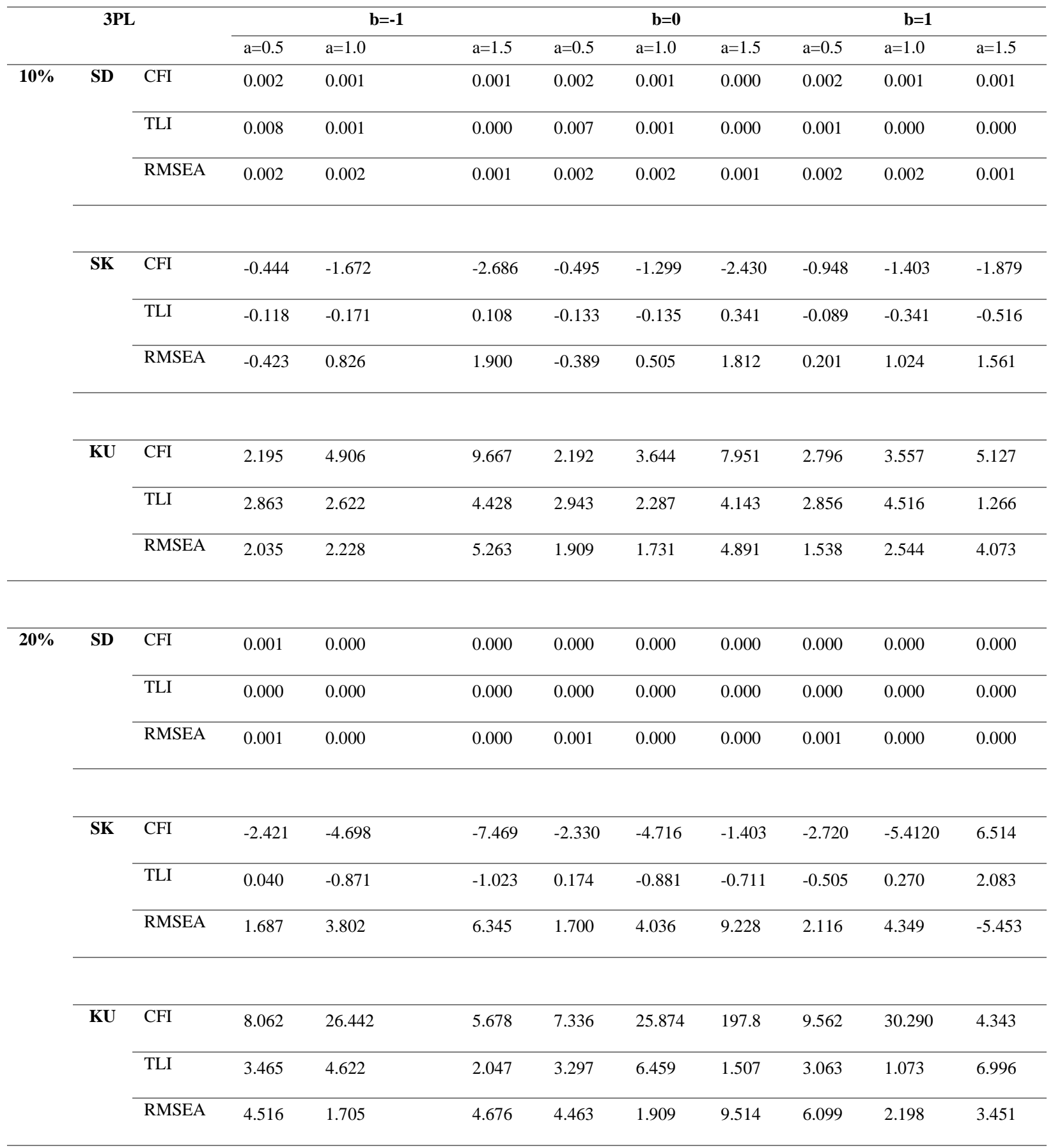


SD of $C F I$ change, $N=2000$, DIF=10\%, IRT=3PL

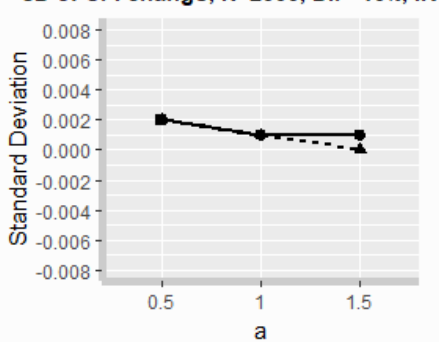

SK of CFI change, $N=2000$, DIF $=10 \%$, IRT $=3 P L$

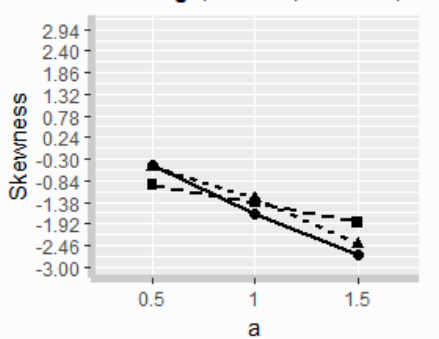

KU of CFI change, $\mathrm{N}=2000$, DIF=10\%, IRT=3PL
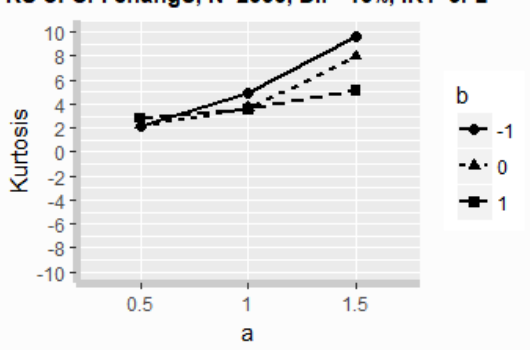

SD of TLI change, $N=2000$, DIF=10\%, IRT=3PL
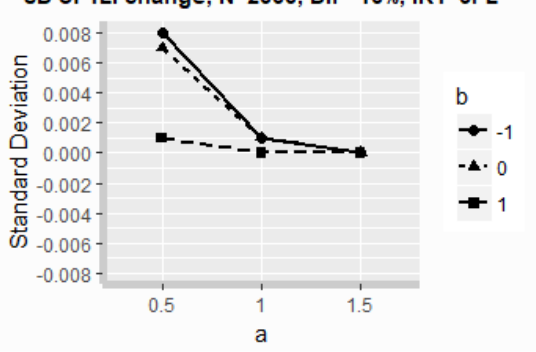

SD of RMSEA change, $N=2000$, DIF $=10 \%, I R T=3 P L$

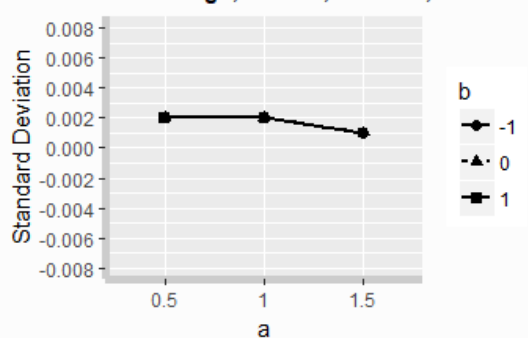

SK of TLI change, $N=2000$, DIF=10\%, IRT=3PL

SK of RMSEA change, $N=2000$, DIF $=10 \%$, IRT $=3 P L$
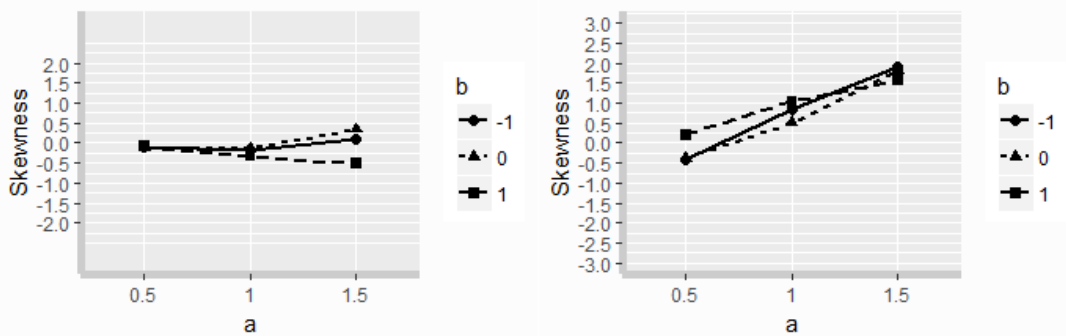

KU of TLI change, $N=2000$, DIF $=10 \%, I R T=3 P L$

KU of RMSEA change, $\mathrm{N}=2000$, DIF= $10 \%$, IRT=3PL

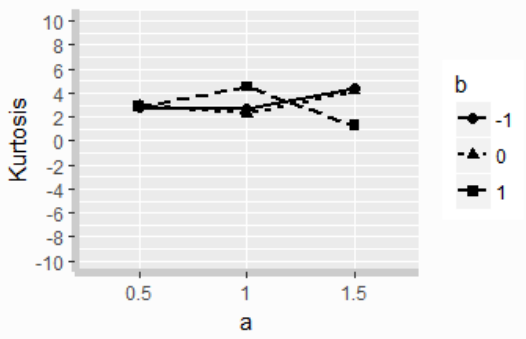

Figure 4.15. Dispersion statistics ( $\mathrm{SD}=$ standard deviation, $\mathrm{SK}=$ skewness, $\mathrm{KU}=$ kurtosis) for the distributions of the change in the CFI, TLI, and the RMSEA fit indices at scalar level across simulation conditions for $\mathrm{N}=2000, \mathrm{IRT}=3 \mathrm{PL}, \mathrm{g}$ DIF percentage $=10 \%$ 

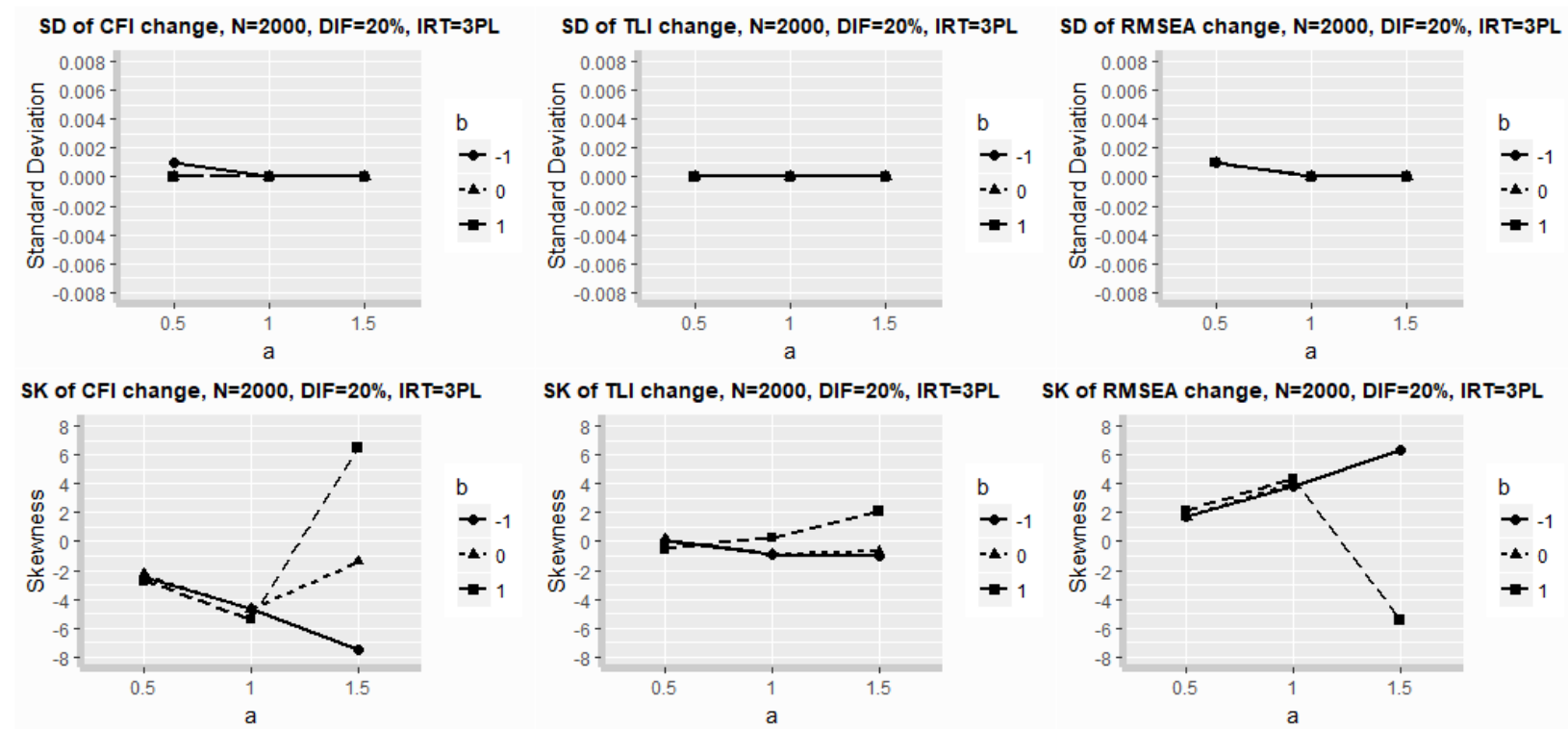

KU of CFI change, $N=2000$, DIF=20\%, IRT=3PL

KU of TLI change, $\mathbf{N}=\mathbf{2 0 0 0}$, DIF=20\%, IR T=3PL

KU of RMSEA change, $\mathrm{N}=\mathbf{2 0 0 0}$, DIF $=20 \%$, IRT $=3 \mathrm{PL}$
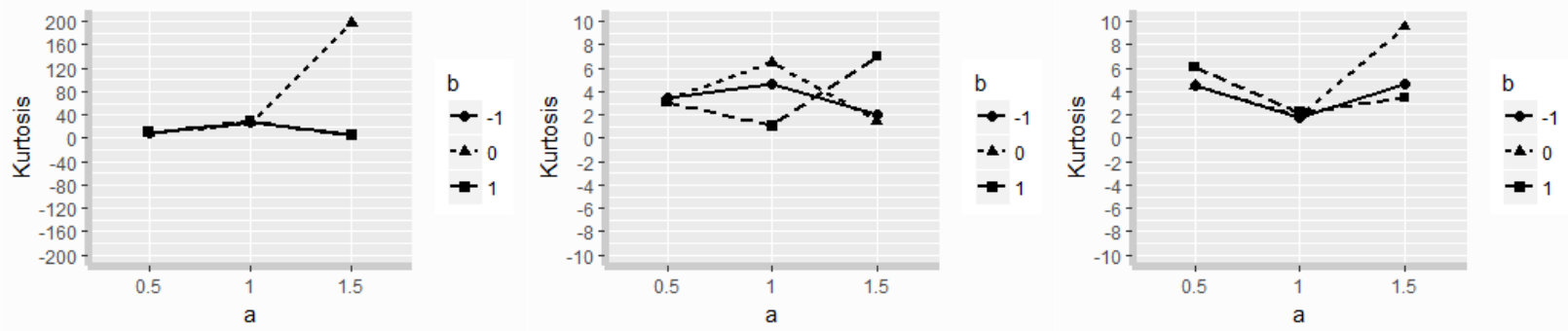

Figure 4.16. Dispersion statistics ( $\mathrm{SD}=$ standard deviation, $\mathrm{SK}=$ skewness, $\mathrm{KU}=$ kurtosis $)$ for the distributions of the change in the CFI, TLI, and the RMSEA fit indices at scalar level across simulation conditions for $\mathrm{N}=2000, \mathrm{IRT}=3 \mathrm{PL}$, DIF percentage $=20 \%$

Table 4.15 and Figures 4.15 and 4.16 show that the standard deviation values are close to zero for all fit indices. As for the skewness, the absolute values increase as the $a$ parameter value increases; deviation from normality are more noticeable in the CFI than the TLI. Kurtosis also increases in all fit indices from lower to higher $a$ parameter value. 
Table 4.16

Distribution statistics Standard Deviation (SD), Skewness (SK), and Kurtosis (KU) for change in CFI, TLI, and RMSEA at scalar level across conditions for $N=2000, I R T=2 P L$.

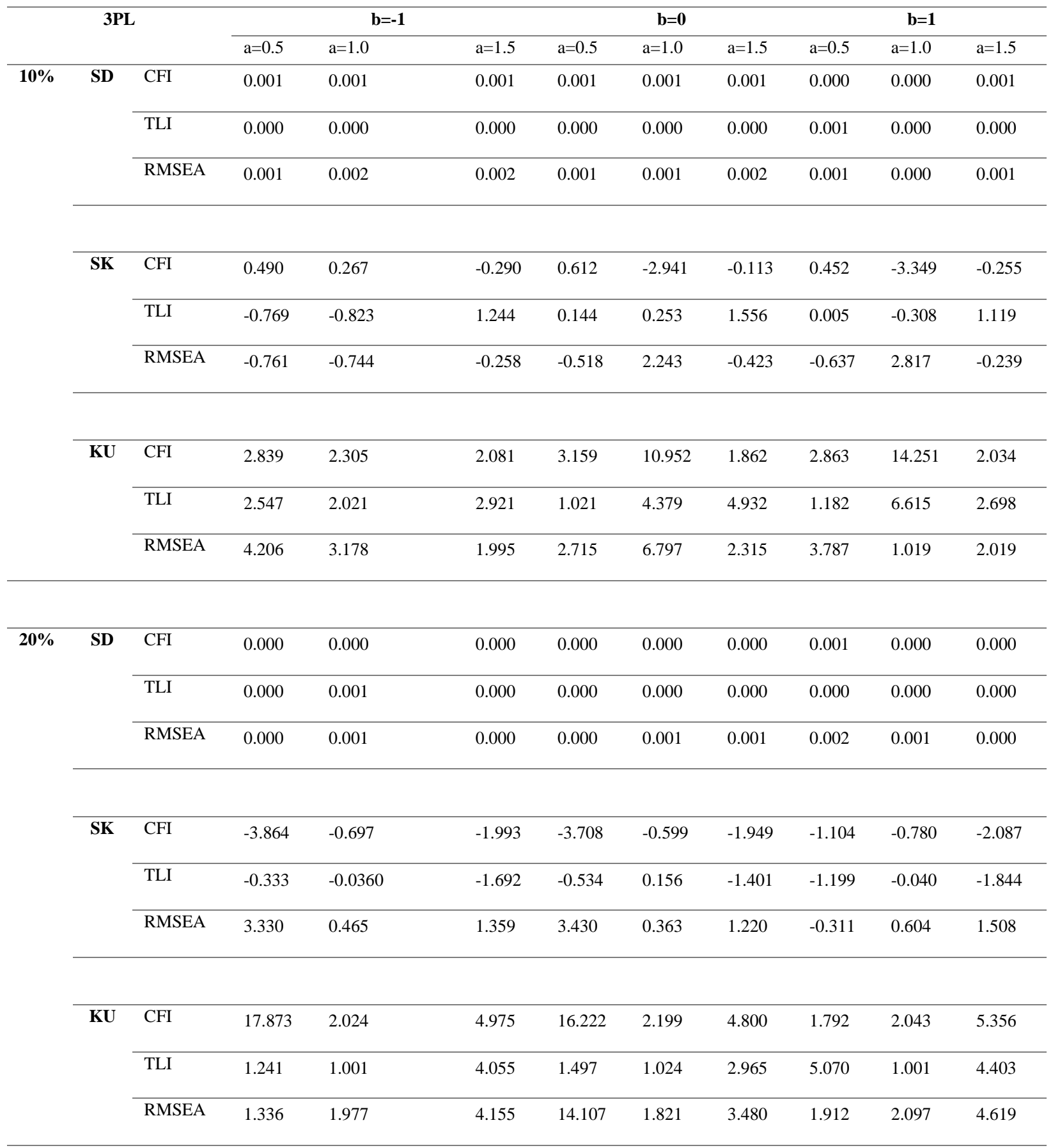


SD of CFI change, $\mathrm{N}=\mathbf{2 0 0 0}$, DIF=10\%, IRT=2PL

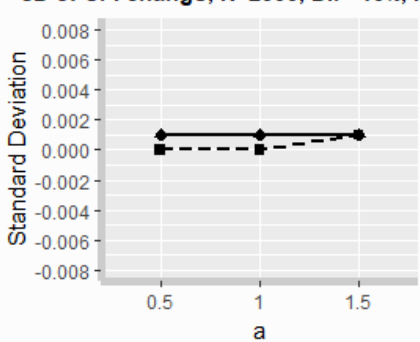

SK of CFI change, $N=2000, D I F=10 \%, I R T=2 P L$

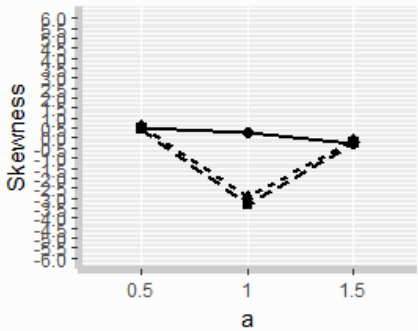

$\mathrm{KU}$ of $\mathrm{CFI}$ change, $\mathrm{N}=\mathbf{2 0 0 0}$, DIF=10\%, IRT=2PL

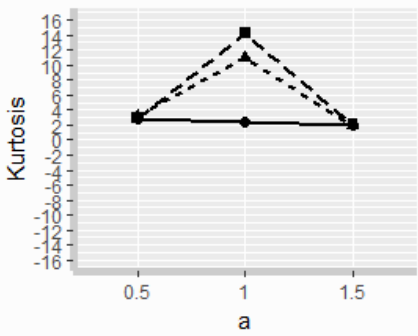

SD of $\mathrm{TLI}$ change, $\mathrm{N}=\mathbf{2 0 0 0 ,}$, DIF=10\%, IRT=2PL

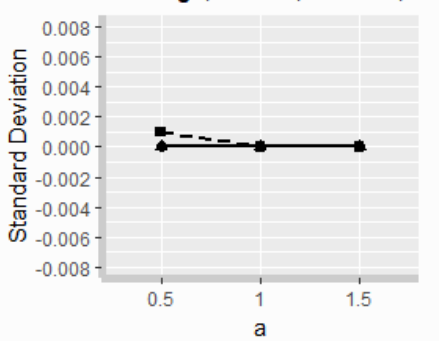

SK of $\mathrm{TLI}$ change, $\mathrm{N}=2000$, DIF=10\%, IRT=2PL
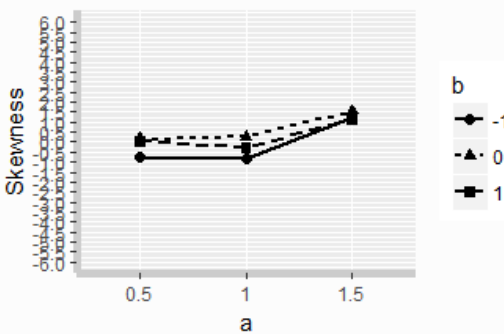

$\mathrm{KU}$ of $\mathrm{TLI}$ change, $\mathrm{N}=\mathbf{2 0 0 0 ,}$ DIF $=10 \%, \mathrm{IRT}=2 \mathrm{PL}$

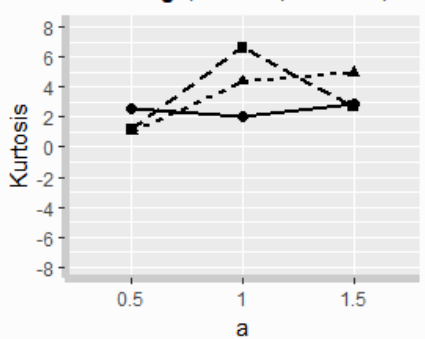

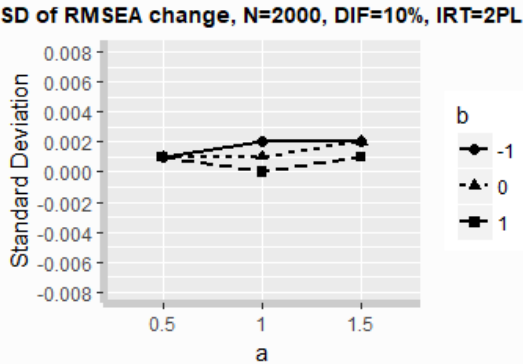

SK of RMSEA change, $N=2000$, DIF $=10 \%$, IRT $=2 P L$

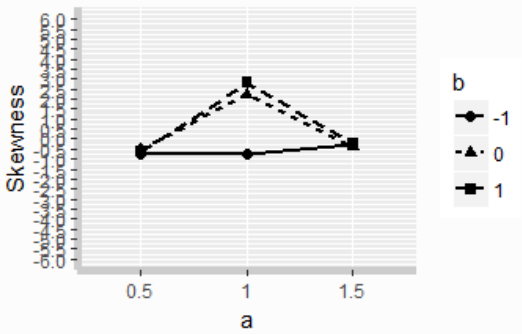

KU of RMSEA change, N=2000, DIF $=10 \%$, IRT $=2 P L$

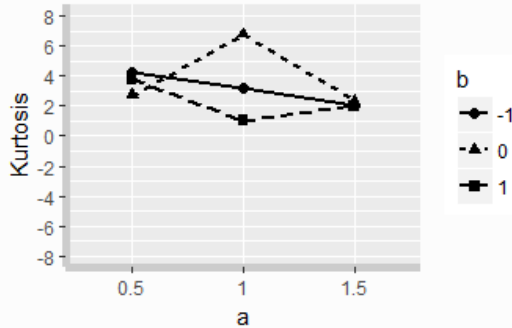

Figure 4.17. Dispersion statistics ( $\mathrm{SD}=$ standard deviation, $\mathrm{SK}=$ skewness, $\mathrm{KU}=$ kurtosis) for the distributions of the change in the CFI, TLI, and the RMSEA fit indices at scalar level across simulation conditions for $\mathrm{N}=2000, \mathrm{IRT}=2 \mathrm{PL}$, DIF percentage $=10 \%$ 

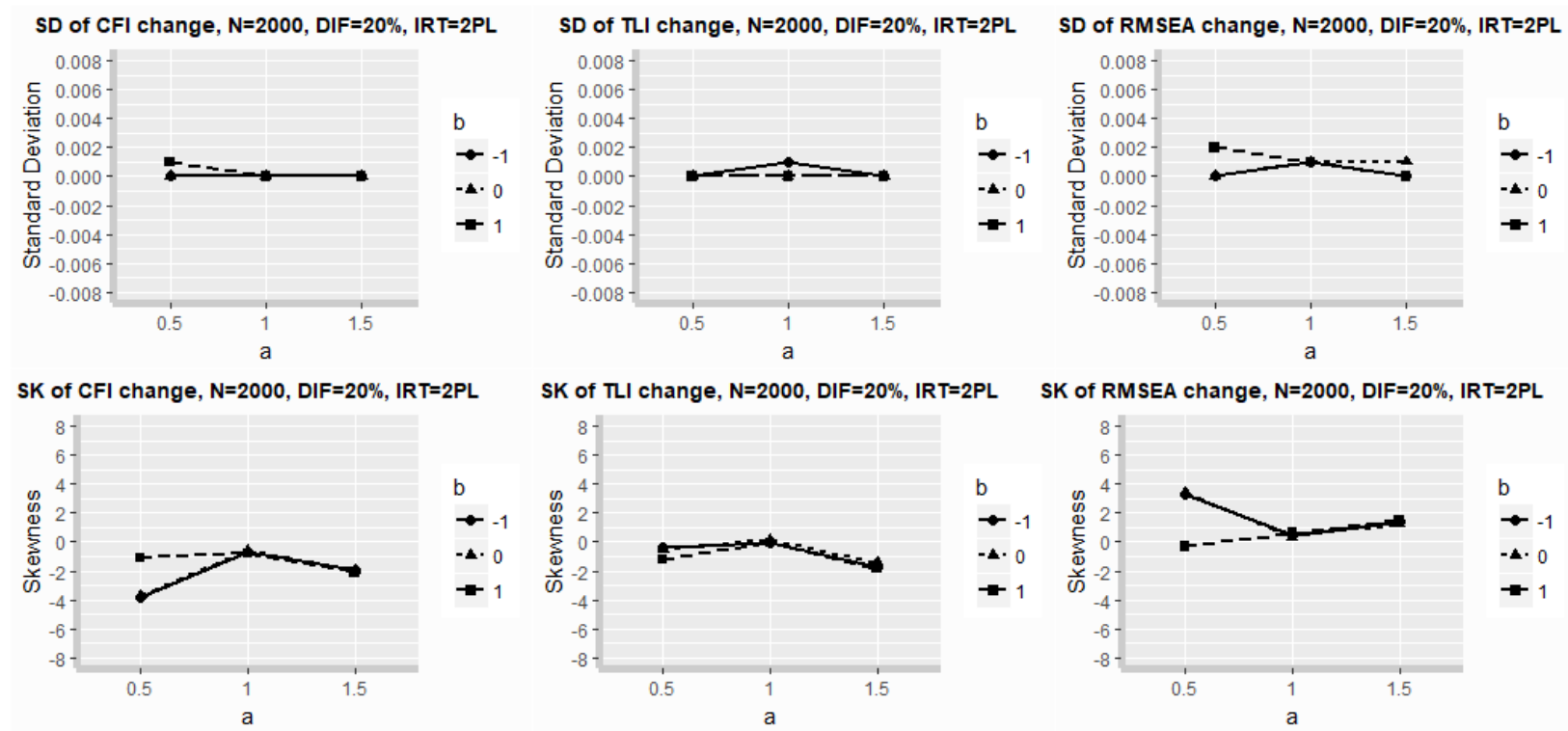

KU of CFI change, $N=2000$, DIF=20\%, IRT=2PL

KU of TLI change, $\mathrm{N}=2000$, DIF=20\%, IR T=2PL

KU of RMSEA change, $\mathrm{N}=2000$, DIF $=20 \%$, IRT=2PL
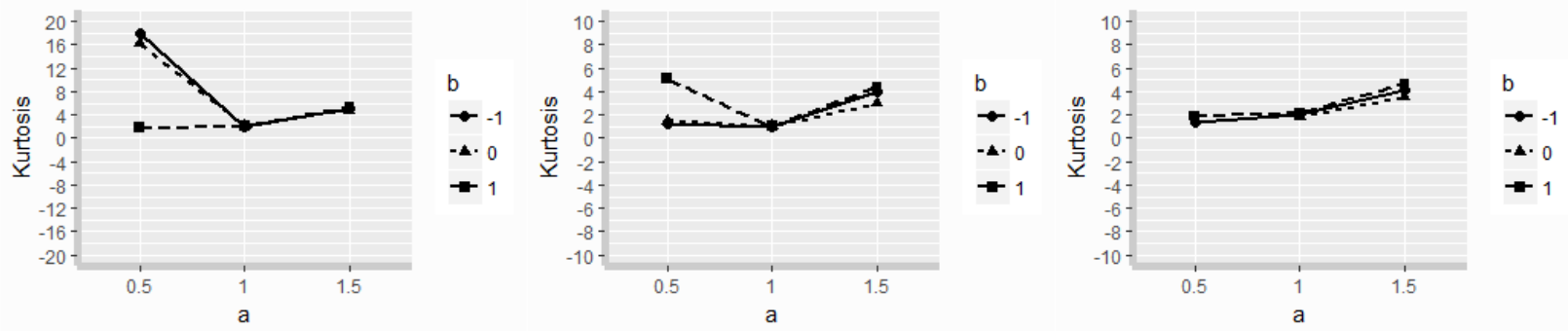

Figure 4.18. Dispersion statistics ( $\mathrm{SD}=$ standard deviation, $\mathrm{SK}=$ skewness, $\mathrm{KU}=$ kurtosis) for the distributions of the change in the CFI, TLI, and the RMSEA fit indices at scalar level across simulation conditions for $\mathrm{N}=2000$, IRT $=2 \mathrm{PL}$, DIF percentage $=20 \%$

For the 2PL model results in Table 4.16 and Figures 4.17 and 4.18, we can see that the standard deviation is close to zero for all fit measures. Skewness is highest when the $a=1$ and $b=1$ for the CFI and the RMSEA. Overall, there is no pattern in the values of the skewness except in the TLI, where its absolute value increases over the $a$ parameters and the b values. The kurtosis values show no pattern either, except that for the most part, they are close to normality. 
Plausible explanations for and interpretation of the simulation results in the context of educational measurement are presented in Chapter Five, along with suggestions for future research. 


\section{CHAPTER 5}

\section{DISCUSSION}

The present simulation study aimed to investigate whether the proposed fit indices in the current literature to assess measurement invariance are appropriate for evaluating the meaningfulness of the lack of invariance in educational assessment data. The present research is different from the previous studies in several ways, including operational context (educational assessment) and parameter estimation method (using robust WLS). However, the most distinguishing difference is that the present research has taken into account the consequences of lack of invariance in terms of a nonignorable impact on total raw scores by constraining the scaled scores (i.e. IRT TTC scores) to differ by 0.5 units in the reference and focal groups. The rationale for the selection of a TCC difference of 0.5 is that such a difference would result in a one-point difference in raw score due to rounding error.

Dichotomous data were simulated using IRT models to represent item responses on largescale assessments. The magnitude of a lack of measurement invariance for the suspected items were created to produce a minimally meaningful difference in the TCCs between the groups - it was argued that a difference of 0.5 in the TCCs between the reference and focal groups represented an interesting difference. MG-CFA was applied to the data to determine if the change in the fit indices (e.g., CFI) was consistent across factors such as test length and item parameter values. In the CFA framework, a commonly suggested criterion used to evaluate measurement invariance is a change in the CFI (or TLI) $<0.01$ (Cheung \& Rensvold, 2002); that is, if the CFI for the invariant model is within 0.01 , then the lack of invariance is considered trivial. This criterion, however, was derived from situations where items were not intended for educational measurement. 
In this study, the CFI, TLI, and the RMSEA fit indices were examined in the multiplegroup confirmatory factor analysis (MG-CFA) framework to investigate their behavior in the presence of non-negligible measurement invariance induced by 0.5 difference in test characteristic curve (TCC) between the focal and the reference groups on a unidimensional IRTcalibrated test with 40 dichotomous items. In other words, the purpose of the present study was to determine if the proposed cutoff value of 0.01 is appropriate in an educational assessment context. In addition, the simulation study investigated the effect of DIF percentage, IRT models, the $a$ and $b$ parameter values, and the sample size on the change in the CFI, TLI, and the RMSEA model fit indices. Overall, five factors and 108 conditions were simulated and investigated.

The results of the study showed that although the MH procedure detected the presence of DIF items, using the practical effect size criterion proposed by Cheung and Rensvold (2002) for the CFI and TLI fit indices failed to support the inference that the lack of invariance was nonnegligible (i.e. the simulated 0.5 difference between the focal and the reference groups). In other words, one cannot rely on the proposed cutoff value in all contexts and under all conditions, as was ostensibly suggested in the results of the present study. Although MH procedure confirmed the presence of DIF, the cutoff value used in practice could not be used to determine a non-trivial LOI because practically all cutoff values in this study were below the 0.01 criterion despite violation of invariance at the scalar level.

One explanation could be the small effect from the differences between the values of the $b$ parameters in the focal and the reference groups. Because the difference between the $b$ parameters of the DIF items in the reference and focal groups was dependent on the value of the $a$ parameter, the occurrence of a few DIF items when the $a$ parameters are high may not affect 
the summary fit indices in the CFA analysis. In the CFA framework, the fit indices are summary statistics and may be influenced by the majority of the item parameters to the effect of missing on the performance of some DIF items. This phenomenon is seen in this study, where the MH procedure on the individual items can detect the DIF items while summary statistics such as the CFI, TLI, and the RMSEA seem to fail to indicate the characteristics of the study items if the flagging criterion used is that of the Cheung and Rensvold (2002) of $\Delta C F I>0.01$. In other words, the changes in the CFI, TLI, and the RMSEA fit indices are not universal and uniform under different model and operation conditions. Therefore, a cutoff value based on summary statistics, such as CFI, TLI, and RMSEA, needs to be determined in the specific measurement context and preferably complimented by other summary statistics and also item level DIF measures, such MH test, logistic regression and IRT-based DIF indices.

In Table 1 below we can see that as the $a$ parameter increased, the difference between the $b$ parameter values in the focal and the reference groups decreased. One may argue for the compensatory effect of the $a$ parameter on decreasing the DIF impact. As is stated in the literature, increased $a$ parameter value leads to increased item fit and hence increased model fit.

Table 1 . The $b$ parameter values used to obtain LOI items in the focal group.

\begin{tabular}{cccccr} 
& & \multicolumn{2}{c}{ 2PL Model } & \multicolumn{2}{c}{ 3PL Model } \\
$a_{\text {Reference }}=a_{\text {Focal }}$ & $b_{\text {Reference }}$ & $b_{\text {Focal }}$ & $b_{\text {Focal }}$ & $b_{\text {Focal }}$ & $b_{\text {Focal }}$ \\
& -1.00 & -2.000 & -1.501 & -2.263 & -1.630 \\
0.5 & 1.006 LOI & $20 \%$ LOI & $10 \%$ LOI & $20 \%$ LOI \\
& 0.00 & 1.006 & 0.501 & 1.260 & 0.630 \\
& 1.00 & -0.006 & 1.501 & 2.263 & 1.630
\end{tabular}




$\begin{array}{cccccc} & -1.00 & -1.502 & -0.750 & -1.629 & -0.687 \\ 1.0 & 0.00 & 0.503 & 0.250 & 0.631 & 0.313 \\ & 1.00 & 1.502 & 1.250 & 1.629 & 1.313 \\ 1.5 & -1.00 & -0.664 & -1.167 & -0.579 & -1.209 \\ & 0.00 & 0.335 & 0.167 & 0.420 & 0.209 \\ & 1.00 & 0.664 & 1.167 & 0.579 & 1.209\end{array}$

\section{Implications for Practice}

The present study attempted to examine the appropriateness of using the established cutoff value of delta CFI/TLI < 0.01 (Cheung \& Rensvold, 2002) in the educational assessment contexts because the foregoing threshold was proposed for application outside educational measurement and a nonspecific measurement setting. The major difference in the design of the present study compared with previous research was introducing non-negligible consequence to a noninvariant test (in terms of a minimum LOI of 0.5 points on the IRT TCC scale between the focal and the reference groups) to evaluate if the purported cutoff value of 0.01 by Cheung and Rensvold (2002) is appropriate to use for detecting LOI in a dichotomous-items educational test usng the MG-CFA method of testing for scalar invariance. According to the results obtained from the study, the cutoff value of 0.01 unit change in CFI/TLI is not an appropriate value for dichotomous items that are calibrated through an IRT 2PL or 3PL model. Therefore, practitioners are advised to consult complimentary measures, such as $\mathrm{MH}$ procedure, logistic regression, or IRT-based methods to evaluate measurement invariance of their tests or instruments. In addition to these sample-based procedures, to obtain cutoff values, simulation 
can also be conducted from the existing distribution to obtain a sample-specific cutoff criterion to determine measurement invariance.

A theoretical contribution of the present study is that summary fit indices such as CFI, TLI, or RMSEA may not capture small to moderate DIF values among a set of items that may be highly similar in their item parameters. In such contexts, item-level DIF indicators (such as IRTbased or CTT-based measures) are recommended. This is especially important in the pilot stage of item selection, as some DIF items may sift through the flagging process.

\section{Future Research}

The present study can be expanded in several ways. In the present study, the nonnegligible difference in the TCC between the reference and the focus groups was set at 0.5 . At this $\triangle \mathrm{TCC}$ value, the fit indices criteria (particularly the changes in CFI and the TLI) failed to detect fit deterioration across the simulated DIF items. In other words, the CFI, TLI, and RMSEA cutoff values established and used in practice were not sensitive enough to reflect LOI of magnitude $0.5 \Delta \mathrm{TCC}$. Therefore, one interesting factor to take into account in a future study would be to introduce different magnitudes of $\triangle \mathrm{TCC}$ (e.g. 1.0, 1.5) for DIF simulation purpose and then investigate if the cutoff value of CFI, TLI, and RMSEA currently in use would be appropriate for such overall DIF magnitudes.

Another factor that may be interesting to investigate is the DIF patterns. In the present study, uniform DIF was simulated to study the behavior of the fit indices and the adequacy of the cutoff value in detecting measurement invariance. An advantage of using the IRT estimation framework in this study is that non-uniform DIF patterns can be easily simulated and studied with respect to their effect on model fit indicators. In addition, mixed DIF patterns and their 
proportion in a set of items can also be simulated and added to the model complexity to evaluate the appropriateness of the present CFI, TLI, and RMSEA criteria in detecting violation of measurement invariance.

In the present study, the number of items was fixed at 40 items. However, different contexts may require different number of items. For instance, in computerized adaptive testing, the number of items may vary across test takers (non-fixed CAT). In larger-scale settings, such as the SAT test, there are even larger number of items administered. Therefore, the number of items can be studied as an additional factor to see if it can alone or in combination with other factors impact how the different fit indices capture the lack of invariance in a set of items. The result of such a study would be more practically generalizable because it addresses a wider context of use in educational measurement.

Items used in the present study were dichotomous. It would be interesting to investigate how the results would be affected if the items were polytomous and estimated through IRT model. Because in polytomous items more than one $b$ parameter value is estimated, therefore in simulating DIF items and defining a minimally interesting DIF impact different strategies need to be adopted. In the polytomous case, the TCC is calculated as in the dichotomous case but with an additional step. While in dichotomous IRT, the TCC is the sum of the ICC's, in polytomous IRT the TCC is the sum of the summed ICC of individual categories. In other words, the ICC of an item is first obtained by the sum of individual parameter estimates of the thresholds, and then these sums are added to get the TCC for the entire test. So, in conducting a research similar to the present one but on polytomous items, the TCC will be not only a function of simulated thresholds, but also the number of items. This may cause a difficulty if the minimal DIF impact is kept small because one polytomous item could produce that impact and when the study 
condition requires more than one simulated DIF items, the degree of DIF in thresholds may need to be reduced to achieve the minimally interesting DIF impact. 


\section{REFERENCES}

American Educational Research Association (AERA), American Psychological Association (APA), \& National Council on Measurement in Education (2014). Standards for educational and psychological testing. Washington, D.C: AERA.

Anderson, J. C., \& Gerbing, D. W. (1988). Structural equation modeling in practice: A review and recommended two-step approach. Psychological Bulletin, 103, 411-423.

Babakus, E., Fergusen, C. E., \& Joreskog, K. G. (1987). The sensitivity of confirmatory maximum likelihood factor analysis to violations of measurement scale and distributional assumptions. Journal of Marketing Research, 24, 222-228.

Bagozzi, R. P., \& Edwards, J. R. (1998). A general approach for representing constructs in organizational research. Organizational Research Methods, 1, 45-87.

Bollen, K. A. (1989). Structural equations with latent variables. New York: John Wiley.

Brannick, M. T. (1995). Critical comments on applying covariance structure modeling. Journal of Organizational Behavior, 16, 201-213.

Brown, T. A. (2006). Confirmatory factor analysis for applied research. New York: The Guilford Press.

Browne, M. W., \& Cudeck, R. (1993). Alternative ways of assessing model fit. In K. A. Bollen \& J. S. Long (Eds.), Testing structural equation models (pp. 136-162). Newbury Park, CA: Sage Publications.

Byrne, B. M. (1989). A primer of LISREL: Basic applications and programming for confirmatory factor analysis models. New York: Springer Verlag. 
Byrne, B. M., Shavelson, R. J., \& Muthen, B. (1989). Testing for the equivalence of factor covariance and mean structures: The issue of partial measurement invariance. Psychological Bulletin, 105, 456-466.

Byrne, B. M., \& Stewart, S. M. (2006). The MACS approach to testing for multigroup invariance of a second-order factor structure: a walk through the process. Structural Equation Modeling, 13, 287-321.

Chau, H., \& Hocevar, D. (1995, April). The effects of number of measured variables on goodness-of-fit in confirmatory factor analysis. Paper presented at the annual meeting of the American Educational Research Association, San Francisco.

Chen, F. F. (2007). Sensitivity of goodness of fit indexes to lack of measurement invariance. Structural Equation Modeling, 14, 464-549.

Cheung, G. W., \& Rensvold, R. B. (2002). Evaluating goodness-of-fit indexes for testing measurement invariance. Structural Equation Modeling: A Multidisciplinary Journal, 9(2), 233-255.

Cumming, G., and Finch, S. (2001). A primer on the understanding, use and calculation of confidence intervals that are based on central and noncentral distributions. Educational and Psychological Measurement, 61, 532-575.

Ding, L., Velicer, W. F., \& Harlow, L., (1995). Effects of estimation methods, number of indicators per factor, and improper solutions on structural equation modeling fit indices. Structural Equation Modeling: A multidisciplinary Journal, 2, 119-144.

Dolan, C. V. (1994). Factor analysis of variables with 2, 3, 5 and 7 response categories: A comparison of categorical variable estimators using simulated data. British Journal of Mathematical and Statistical Psychology, 47, 309-326. 
Fan, X., Thompson, B. \& Wang, L. (1999) Effects of sample size, estimation methods, and model specification on structural equation modeling fit indexes, Structural Equation Modeling: A multidisciplinary Journal, 6:1, 56-83.

Flora, D. B., \& Curran, P. J. (2004). An empirical evaluation of alternative methods of estimation for confirmatory factor analysis with ordinal data. Psychological Methods, 9, 4, 466-491.

Gerbing, D. W., \& Anderson, J. C. (1992). Monte Carlo evaluations of goodness-of-fit indices for structural equation models. Sociological Methods \& Research, 21, 132-160.

Hallquist, M., \& Wiley, J. (2017). Mplus Automation: automating Mplus model estimation and interpretation. R package version 0.6-3. ??

Harrington, D. (2009). Confirmatory factor analysis. New York: Oxford University Press.

Holland, P. W., \& Thayer, D. T. (1988). Differential item performance and the Mantel-Haenszel procedure. In H. Wainer \& H. I. Braun (Eds.), Test validity (pp. 129-145). Hillsdale, NJ: Erlbaum.

Hu, L. and Bentler, P. M. (1999). Cutoff criteria for fit indices in covariance structure analysis: Conventional criteria versus new alternatives. Structural Equation Modeling, A multidisciplinary Journal (1), 1-55.

Jin, Ying (2020). A note on the cutoff values of alternative fit indices to evaluate measurement invariance for ESEM models. International Journal of Behavioral Development 2020, Vol. 44(2) 166-174.

Kenny, D.A. \& McCoach, D. B. (2003). Effect of the number of variables on measures of fit in structural equation modeling. Structural Equation Modeling, A multidisciplinary Journal (10,3), 333-351. 
Kenny, D.A. (2012). Measuring Model Fit. Retrieved from: http://www.davidakenny.net/cm/fit.htm

Kelloway, E. K. (1995). Structural equation modeling in perspective. Journal of Organizational Behavior, 16, 215-224.

Khojasteh, Jam, \& Lo, Wen-Juo. (2015): Investigating the sensitivity of goodness-of-fit indices to detect measurement invariance in a bifactor model. Structural Equation Modeling: A Multidisciplinary Journal, 00: 1-11, 2015.

Kline, R. B. (2011). Principles and practice of structural equation modeling, 3rd ed. New York: The Guilford Press.

Little, T. D. (1997). Mean and covariance structure (MACS) analyses of cross-cultural data: Practical and theoretical issues. Multivariate Behavioral Research, 32, 53-76.

Marsh, H. W., Hau, K. T., Balla, J. R., \& Grayson, D. (1988). Is more ever too much? The number of indicators per factor in confirmatory factor analysis. Multivariate Behavioral Research, 33, 181-222.

McDonald, R. P., \& Marsh, H. W. (1990). Choosing a multivariate model: Noncentrality and goodness-of-fit, Psychological Bulletin 107: 247-255.

Meade, A. W., Johnson, E. C., \& Braddy, P. W. (2008). Power and sensitivity of alternative fit indices in tests of measurement invariance. Journal of Applied Psychology, 93, 568-592.

Meredith, W. (1965). Some results based on a general stochastic model for mental tests. Psychometrika, 30, 419-440.

Millsap, R. E. (2011). Statistical approaches to measurement invariance. New York: Routledge.

Millsap, R. E., \& Hartog, S. B. (1988). Alpha, beta, and gamma change in evaluation research: A structural equation approach. Journal of Applied Psychology, 73, 574-584. 
Millsap, R. E., \& Olivera-Aguilar, M. (2012). Investigating measurement invariance using confirmatory factor analysis. In R. H. Hoyle (Ed.). Handbook of structural equation modeling, (pp. 380-392). New York: The Guilford Press.

Mulaik, S. A. (1975). Confirmatory factor analysis. In D. J. Amick \& H. J. Walberg (Eds.), Introductory multivariate analysis (pp. 170-207). Berkley, CA: McCuchan.

Muthén, B., du Toit, S.H.C. \& Spisic, D. (1997). Robust inference using weighted least squares and quadratic estimating equations in latent variable modeling with categorical and continuous outcomes. Technical Report.

Muthen, B. O. (1989). Latent variable modeling in heterogeneous populations. Psychometrika, $54(4), 557-85$.

Raykov, T. \& Marcoulides, G. A. (2006) A first course in structural equation modeling, second edition. Mahwah, New Jersey: Lawrence Erlbaum Associates.

Reise, S. P., Widaman, K. F, \& Pugh, R. H. (1993). Confirmatory factor analysis and item response theory: Two approaches for exploring measurement invariance. Psychological Bulletin, 114, 552-566.

Rigdon, E. E., Ferguson, C. E., Jr. (1991). The performance of the polychoric correlation coefficient and selected fitting functions in confirmatory factor analysis with ordinal data. Journal of Marketing Research, 28, 491-7.

Sass, D. A., Schmitt. T. A., \& Marsh, H. W (2014). Evaluating model fit with ordered categorical data within a measurement invariance framework: a comparison of estimators. Structural Equation Modeling: A Multidisciplinary Journal, 21:2, 167-180. 
Schaubroeck, J. \& Green, S. G. (1989). Confirmatory factor analytic procedures for assessing change during organizational entry. Journal of Applied Psychology, 74, 892-900.

Schmitt, N. (1982). The use of the analysis of covariance structures to assess beta and gamma change. Multivariate Behavioral Research, 17, 343-358.

Schmitt, N., \& Kuljanin, G. (2008). Measurement invariance: review of practice and implications. Human Resources Management Review, 18, 210-222.

Steenkamp, J. E. M., \& Baumgartner, H. (1998). Assessing measurement invariance in crossnational consumer research. Journal of Consumer Research, 25, 78-90.

Swaminathan, H., \& Rogers, H. J. (1990). Detecting differential item functioning using logistic regression procedures. Journal of Educational Measurement, 27, 361-370.

Thissen, D., Steinberg, L., \& Gerrard, M. (1986). Beyond group-mean differences: the concept of item bias. Psychological Bulletin, 99, 118-128.

Thissen, D., Steinberg, L., \& Wainer, H. (1988). Use of item response theory in the study of group differences in trace lines. In H. Wainer \& H. Braun (Eds.), Test validity (pp. 147169). Hillsdale, NJ: Erlbaum.

Thissen, D., Steinberg, L., \& Wainer, H. (1993). Detection of differential item functioning using the parameters of item response models. In P. Holland \& H. Wainer (Eds.), Differential item functioning (pp. 67-113). Hillsdale, NJ: Erlbaum.

Tucker, L. R., \& Lewis, C. (1973). A reliability coefficient for maximum likelihood factor analysis. Psychometrica, 38, 1-10.

Vandenberg, R. J. \& Self, R. M. (1993). Assessing newcomers' changing commitment to the organization during the first 6 months of work. Journal of Applied Psychology, 78, 557568. 
Vandenberg, R. J., \& Lance, C. E. (2000). A review and synthesis of the measurement invariance literature: suggestions, practices, and recommendations for organizational research. Organizational Research Methods, 3(1), 4-70.

Vandenberg, R. J. (2002). Toward a further understanding of and improvement in measurement invariance methods and procedures. Organizational Research Methods, 5, 139-158.

Willet, J. B., \& Sayer, A. G. (1994). Using covariance structure analysis to detect correlates and predictors of individual change over time. Psychological Bulletin, 116, 363-381.

Widaman, K. F. \& Reise, S. P. (1997). Exploring the measurement invariance of psychological instruments: applications in substance use domain. In K. J. Bryant, M. Windle, \& S. G. West (Eds), The science of prevention (pp. 281-324). Washington, D.C: American Psychological Association.

Yoon, M., \& Millsap, R. E. (2007). Detecting violations of factorial invariance using data-based specification searches: A Monte Carlo study. Structural Equation Modeling, 14, 435-463. 